\title{
PROBABILISTIC INTERPRETATION OF A SYSTEM OF COUPLED HAMILTON-JACOBI-BELLMAN-ISAACS EQUATIONS*
}

\author{
JUAN LI ${ }^{1}$, WENQIANG LI $^{2}$ AND QINGMENG $\mathrm{WEI}^{3, * *}$
}

\begin{abstract}
By introducing a stochastic differential game whose dynamics and multi-dimensional cost functionals form a multi-dimensional coupled forward-backward stochastic differential equation with jumps, we give a probabilistic interpretation to a system of coupled Hamilton-Jacobi-Bellman-Isaacs equations. For this, we generalize the definition of the lower value function initially defined only for deterministic times $t$ and states $x$ to stopping times $\tau$ and random variables $\eta \in L^{2}\left(\Omega, \mathcal{F}_{\tau}, P ; \mathbb{R}\right)$. The generalization plays a key role in the proof of a strong dynamic programming principle. This strong dynamic programming principle allows us to show that the lower value function is a viscosity solution of our system of multi-dimensional coupled Hamilton-Jacobi-Bellman-Isaacs equations. The uniqueness is obtained for a particular but important case.
\end{abstract}

Mathematics Subject Classification. 49N70, 49L25, 60H10, 93E20.

Received July 28, 2019. Accepted October 19, 2020.

\section{INTRODUCTION}

This paper is devoted to the study of a probabilistic interpretation of the following multi-dimensional coupled system of Hamilton-Jacobi-Bellman-Isaacs (HJBI, for short) equations:

$$
\left\{\begin{aligned}
& \frac{\partial W_{i}}{\partial t}(t, x)+\sup _{u \in U} \inf _{v \in V}\{ b_{i}\left(t, x, W_{i}(t, x), D W_{i}(t, x) \sigma_{i}\left(t, x, W_{i}(t, x), u, v\right), u, v\right) D W_{i}(t, x) \\
&+\frac{1}{2} \operatorname{tr}\left(\sigma_{i} \sigma_{i}^{*}\left(t, x, W_{i}(t, x), u, v\right) D^{2} W_{i}(t, x)\right) \\
&\left.+f_{i}\left(t, x, \mathbf{W}(t, x), D W_{i}(t, x) \sigma_{i}\left(t, x, W_{i}(t, x), u, v\right), u, v\right)\right\}=0, \\
&(t, x) \in[0, T) \times \mathbb{R}, i \in \mathbf{K}, \\
& W_{i}(T, x)=g_{i}(x), x \in \mathbb{R}, i \in \mathbf{K},
\end{aligned}\right.
$$

* The work has been supported in part on one hand by the NSF of P.R. China (No. 11871037, 11971099), National Key R and D Program of China (No. 2018YFA0703900), NSFC-RS (No. 11661130148, NA150344), on the other hand by the Natural Science Foundation of Shandong Province (ZR2017MA015), Doctoral Scientific Research Fund of YantaiUniversity (No. SX17B09), and by the Science and Technology Development Plan Project of Jilin Province (20190103026JH).

Keywords and phrases: Strong dynamic programming principle, coupled FBSDEs with jumps, stochastic differential games, HJBI equations.

${ }^{1}$ School of Mathematics and Statistics, Shandong University, Weihai, Weihai 264209, P.R. China.

2 School of Mathematics and Information Sciences, Yantai University, Yantai 264005, P.R. China.

3 School of Mathematics and Statistics, Northeast Normal University, Changchun 130024, P.R. China.

** Corresponding author: weiqm100@nenu.edu.cn 
where $T>0$ is an arbitrarily fixed finite time horizon, $U$ and $V$ are two compact metric spaces, $k \geq 2$ is an integer, $\mathbf{K}=\{1,2, \cdots k\}$ and

$$
\mathbf{W}(t, x)=\left(W_{1}(t, x), W_{2}(t, x), \ldots, W_{k}(t, x)\right),(t, x) \in[0, T] \times \mathbb{R} .
$$

The precise assumptions on the functions $b_{i}, \sigma_{i}, f_{i}, g_{i}, 1 \leq i \leq K$, will be given in Section 3 . Note that the system of $k$ HJBI equations (1.1) is coupled through the $k$-dimensional solution $\mathbf{W}(t, x)$.

The probabilistic interpretation for partial differential equations (PDEs, for short) has been investigated by many authors, but for such coupled system of PDEs has been studied by few authors. When both $U$ and $V$ are single point sets, Pardoux, Pradeilles and Rao [8] obtained a probabilistic interpretation for the system (1.1) with the help of backward stochastic differential equations (BSDEs, for short) associated to a diffusion jump process. When either $U$ or $V$ is a singleton, the stochastic representation for (1.1) was studied by Buckdahn, Hu [2] by a stochastic control problem. We emphasize that the functions $b_{i}, \sigma_{i}, i \in \mathbf{K}$, in (1.1) also do not depend on the variables $y$ and $z$ in $[2,8]$, and $\operatorname{PDE}(1.1)$ is totally new. On the other hand, for the case $k=1$, the system (1.1) is reduced to a generalized HJBI equation whose probabilistic interpretation has been obtained by $\mathrm{Li}$, Wei [7] using the lower value function of a stochastic differential game problem. The reader is also referred to $[3-5,9,10]$ for the probabilistic interpretation of a HJBI equation and the references therein.

In this paper, we introduce a stochastic differential game problem on a Wiener-Poisson space in order to give the probabilistic interpretation for (1.1) in the general case. Let us be more precise: The dynamics of our stochastic differential games is given by the following coupled FBSDE with jumps

$$
\left\{\begin{aligned}
\mathrm{d} X_{s}^{t, \eta, i ; u, v}= & b_{N_{s}^{t, i}}\left(s, X_{s}^{t, \eta, i ; u, v}, Y_{s}^{t, \eta, i ; u, v}, Z_{s}^{t, \eta, i ; u, v}, u_{s}, v_{s}\right) \mathrm{d} s+\sigma_{N_{s}^{t, i}}\left(s, X_{s}^{t, \eta, i ; u, v}, Y_{s}^{t, \eta, i ; u, v}, u_{s}, v_{s}\right) \mathrm{d} B_{s}, \\
X_{t}^{t, \eta, i ; u, v}= & \eta, \\
\mathrm{d} Y_{s}^{t, \eta, i ; u, v}= & -\tilde{f}_{N_{s}^{t, i}}\left(s, X_{s}^{t, \eta, i ; u, v}, Y_{s}^{t, \eta, i ; u, v}, H_{s}^{t, \eta, i ; u, v}, Z_{s}^{t, \eta, i ; u, v}, u_{s}, v_{s}\right) \mathrm{d} s+\lambda \sum_{l=1}^{k-1} H_{s}^{t, \eta, i ; u, v}(l) \mathrm{d} s \\
& +Z_{s}^{t, \eta, i ; u, v} \mathrm{~d} B_{s}+\sum_{l=1}^{k-1} H_{s}^{t, \eta, i ; u, v}(l) \mathrm{d} \tilde{N}_{s}(l), \quad s \in[t, T], \\
Y_{T}^{t, \eta, i ; u, v}= & g_{N_{T}^{t, i}}\left(X_{T}^{t, \eta, i ; u, v}\right),
\end{aligned}\right.
$$

where $N^{t, i}$ is a $\mathbf{K}$-valued Markov process which will be specified in the next section, and $\tilde{f}_{i}$ is introduced to overcome the difficulties related with the coupling in $(1.1)(i=1, \ldots, k)$. The relationship between $\tilde{f}_{i}$ and $f_{i}$, $i \in \mathbf{K}$, will be given in Section 3. We study the game of the type "strategy against control", so the lower value function is defined as follows:

$$
W_{i}(t, x):=\operatorname{essinf}_{\beta \in \mathcal{B}_{t, T}} \operatorname{esssup}_{u \in \mathcal{U}_{t, T}} Y_{t}^{t, x, i ; u, \beta(u)}, i \in \mathbf{K},(t, x) \in[0, T] \times \mathbb{R},
$$

where $\mathcal{U}_{t, T}$ is the set of admissible controls of player I and $\mathcal{B}_{t, T}$ is the set of nonanticipative strategies for player II; for the precise definitions of $\mathcal{U}_{t, T}$ and $\mathcal{B}_{t, T}$, see the Definitions 3.4 and 3.5, respectively. We first prove that $W_{i}(t, x), 1 \leq i \leq K$, satisfy some regularity properties: They are deterministic, Lipschitz continuous in $x$ and $\frac{1}{2}$-Hölder continuous in $t$. In order to establish the relation between the lower value function (1.3) and the coupled system (1.1), the crucial step is to get the dynamic programming principle (DPP, for short) for $W_{i}(t, x)$, $i \in \mathbf{K}$. However, the classical DPP is not sufficient anymore in our framework, since stopping times are involved in the transformation between $\tilde{f}_{N_{s}^{t, i}}$ and $\tilde{f}_{i}, i \in \mathbf{K}$, in the proof of the existence of a viscosity solution. Then we consider the DPP (Thm. 3.17) in strong sense, with the help of the notion of the backward stochastic semigroup introduced by Peng in [10]. It is worth mentioning that this strong DPP is far from being obvious and its proof is not a standard generalization of the classical DPP. The key step is to deduce an important equality

$$
W_{N_{\tau}^{t, i}}(\tau, \eta)=\operatorname{essinf}_{\beta \in \mathcal{B}_{\tau, T}} \operatorname{esssup}_{u \in \mathcal{U}_{\tau, T}} Y_{\tau}^{\tau, \eta, N_{\tau}^{t, i} ; u, \beta(u)}, i \in \mathbf{K},
$$


(see Prop. 3.16) which is the extension of the definition for the lower value function (which is originally defined only for deterministic times $t$ and states $x)$ to stopping times $\tau$ and random variables $\eta \in L^{2}\left(\Omega, \mathcal{F}_{\tau}, P ; \mathbb{R}\right)$. This generalization needs a series of auxiliary results, for which we provide the details in Section 3. The DPP will allow to prove that the lower value function $\mathbf{W}(t, x)=\left(W_{1}(t, x), W_{2}(t, x), \ldots, W_{k}(t, x)\right)$ defined by $(1.3)$ is a viscosity solution of the system of HJBI equations (1.1). Moreover, the uniqueness of the viscosity solution is also proved when the coefficients $\sigma_{i}, i \in \mathbf{K}$, in (1.1)-(1.2) are independent of $y$.

In addition, our stochastic differential game problem has the upper value function $U_{i}(t, x):=\operatorname{esssup}_{\alpha \in \mathcal{A}_{t, T}}^{\operatorname{essinf}} v \in \mathcal{V}_{t, T}$ $Y_{t}^{t, x, i ; \alpha(v), v}, i \in \mathbf{K}$ (for the notations, see the Defs. 3.4 and 3.5) and it has properties similar to $W_{i}(t, x), i \in \mathbf{K}$. To avoid repetitions, we only state the main results for $U_{i}(t, x)$ : The upper value function $U_{i}(t, x), i \in \mathbf{K}$, of the stochastic differential game is the (unique) viscosity solution of the following coupled HJBI equation:

$$
\left\{\begin{aligned}
\frac{\partial U_{i}}{\partial t}(t, x)+\inf _{v \in V} \sup _{u \in U}\{ & b_{i}\left(t, x, U_{i}(t, x), D U_{i}(t, x) \sigma_{i}\left(t, x, U_{i}(t, x), u, v\right), u, v\right) D U_{i}(t, x) \\
& +\frac{1}{2} \operatorname{tr}\left(\sigma_{i} \sigma_{i}^{*}\left(t, x, U_{i}(t, x), u, v\right) D^{2} U_{i}(t, x)\right) \\
& \left.+f_{i}\left(t, x, \mathbf{U}(t, x), D U_{i}(t, x) \sigma_{i}\left(t, x, U_{i}(t, x), u, v\right), u, v\right)\right\}=0, \\
U_{i}(T, x)=g_{i}(x), &
\end{aligned}\right.
$$

where $\mathbf{U}(t, x)=\left(U_{1}(t, x), U_{2}(t, x), \ldots, U_{k}(t, x)\right),(t, x) \in[0, T] \times \mathbb{R}, i \in \mathbf{K}=\{1,2, \ldots k\}, k \geq 2$. As a byproduct, we obtain that the stochastic differential game has a value under the well-known Isaacs' condition.

Our paper is organized as follows. In Section 2, we introduce the underlying probability space and some notations, and we recall the preliminaries of BSDEs with jumps. Section 3 is devoted to the formulation of the stochastic differential games and the study of the properties of the lower value functions $W_{i}, i \in \mathbf{K}$. Moreover, we show that they satisfy the strong dynamic programming principle. In Section 4, we present the details of the relationship between the lower value functions of the stochastic differential games and the coupled HJBI equations. In the appendix we give the detailed illustration on the comparison theorem for FBSDEs with jumps and the proof of Theorem 3.17, respectively.

\section{Preliminaries}

\subsection{Some notations}

Let $\mathbf{K}=\{1,2, \ldots, k\}$, where $k \geq 2$ is a given integer. Let $(\Omega, \mathcal{F}, P)$ be the completed product of the probability spaces $\left(\Omega_{1}, \mathcal{F}_{1}, P_{1}\right)$ and $\left(\Omega_{2}, \mathcal{F}_{2}, P_{2}\right)$, where

- $\left(\Omega_{1}, \mathcal{F}_{1}, P_{1}\right)$ is a classical Wiener space, namely $\Omega_{1}=C_{0}\left(\mathbb{R} ; \mathbb{R}^{d}\right)$ is the set of all continuous functions from $\mathbb{R}$ to $\mathbb{R}^{d}$ with value 0 at time $0, \mathcal{F}_{1}$ is the completed Borel $\sigma$-field on $\Omega_{1}, P_{1}$ is the Wiener measure such that the canonical processes $B_{s}(\omega)=\omega(s), s \in \mathbb{R}_{+}, \omega \in \Omega_{1}$ and $B_{-s}(\omega)=\omega(-s), s \in \mathbb{R}_{+}, \omega \in \Omega_{1}$, are two independent $d$-dimensional Brownian motions. We denote by $\mathbb{F}^{B}=\left(\mathcal{F}_{s}^{B}\right)_{s \geq 0}$ the completed filtration generated by the Brownian motion $B$, i.e., $\mathcal{F}_{s}^{B}=\sigma\left\{B_{r}, r \in(-\infty, s]\right\} \vee \mathcal{N}_{P_{1}}$, where $\mathcal{N}_{P_{1}}$ is the collection of null-sets of $P_{1}$.

- $\left(\Omega_{2}, \mathcal{F}_{2}, P_{2}\right)$ is a Poisson space: A point function $p: D_{p} \subset \mathbb{R} \rightarrow \mathbf{L}$ is a map defined on a countable subset $D_{p}$ of the real line $\mathbb{R}$, where $\mathbf{L}=\mathbf{K}-\{k\}$ is equipped with the $\sigma$-field $\mathcal{L}$ of all subsets of $\mathbf{L} ; \Omega_{2}$ is the set of all point functions $p$ on $\mathbf{L}$.

The counting measure $N$ on $\mathbb{R} \times \mathbf{L}$ at $p \in \Omega_{2}$ is defined as follows

$$
N(p,(s, t] \times \Delta)=\sharp\left\{r \in D_{p} \cap(s, t]: p(r) \in \Delta\right\}, \Delta \in \mathcal{L}, s, t \in \mathbb{R}, s<t,
$$

where $\sharp$ denotes the cardinal number of elements of the set. We identify the point function $p$ with $N(p, \cdot)$. The $\sigma$-field $\mathcal{F}_{2}$ is defined as the smallest one on $\Omega_{2}$ with respect to which the coordinate mapping $p \rightarrow$ $N(p,(s, t] \times \Delta), \Delta \in \mathcal{L}, s, t \in \mathbb{R}, s<t$, is measurable. For fixed $\lambda>0$, we consider the probability 
measure $P_{2}$ on $\left(\Omega_{2}, \mathcal{F}_{2}\right)$ such that the coordinate measure $N$ becomes a Poisson random measure with the compensator $\hat{N}((s, t] \times\{l\})=(t-s) \lambda \sum_{n=1}^{k-1} \delta_{n}(l)=\lambda(t-s), l \in \mathbf{L}$. Denoting by $\{\tilde{N}((s, t] \times\{l\})\}_{s \leq t}$ the Poisson martingale measure defined as $\{(N-\hat{N})((s, t] \times\{l\})\}_{s \leq t}=\{N((s, t] \times\{l\})-\lambda(t-s)\}_{s \leq t}$, for all $l \in \mathbf{L}$, we recall that the processes $\{\tilde{N}((s, t] \times\{l\})\}_{0 \leq s \leq t \leq T}, 1 \leq l \leq k-1$, are independent. Above $\delta_{n}(\cdot)$ is the Dirac measure over $\mathbf{L}$, that is, $\delta_{n}(l)=1$, if $l=n$, and $\delta_{n}(l)=0$, otherwise. By defining $\dot{\mathcal{F}}_{t}^{N}:=\sigma\{N((s, r] \times \Delta):-\infty<s \leq r \leq t, \Delta \in \mathcal{L}\}, t \geq 0$, we get the filtration generated by the Poisson random measure $N$ is $\left(\mathcal{F}_{t}^{N}\right)_{t \geq 0}: \mathcal{F}_{t}^{N}=\left(\bigcap_{s>t} \dot{\mathcal{F}}_{s}^{N}\right) \vee \mathcal{N}_{P_{2}}, t \geq 0$.

Finally, put $\Omega=\Omega_{1} \times \Omega_{2}, P=P_{1} \otimes P_{2}, \mathcal{F}=\left(\mathcal{F}_{1} \otimes \mathcal{F}_{2}\right) \vee \mathcal{N}_{P}$, where $\mathcal{F}$ is completed with respect to $P$. We denote by $\mathbb{F}=\left(\mathcal{F}_{t}\right)_{t \geq 0}$ the filtration generated by Brownian motion $B$ and Poisson random measure $N$, and augmented by all $P$-null sets, i.e., $\mathcal{F}_{t}=\left(\mathcal{F}_{t}^{B} \otimes \mathcal{F}_{t}^{N}\right) \vee \mathcal{N}_{P}, t \geq 0$.

For $0 \leq t \leq s \leq T, i \in \mathbf{K}$ and $l \in \mathbf{L}$, we put $N_{s}=N((0, s] \times \mathbf{L}), N_{s}(l)=N((0, s] \times\{l\})$ and $\tilde{N}_{s}(l)=N_{s}(l)-\lambda s$. We introduce a $\mathbf{K}$-valued Markov process $N_{s}^{t, i}$ as $N_{s}^{t, i}=\left(i+\sum_{l=1}^{k-1} l N((t, s] \times\{l\})\right) \bmod (k)$, where $(j) \bmod (k)$ is identified with $j^{\prime} \in \mathbf{K}$ such that $j-j^{\prime}$ is a multiple of $k$, for any given natural $j \geq 1$.

Let $T>0$ be a finite time horizon. We introduce the following spaces of processes:

$-\mathcal{S}^{2}(t, T ; \mathbb{R}):=\left\{\psi \mid \psi: \Omega \times[t, T] \rightarrow \mathbb{R}\right.$ is an $\left(\mathcal{F}_{t}\right)_{t \geq 0}$-adapted càdlàg process, and $\left.\mathbb{E}\left[\sup _{s \in[t, T]}\left|\psi_{s}\right|^{2}\right]<+\infty\right\} ;$

$-\mathcal{M}^{2}\left(t, T ; \mathbb{R}^{d}\right):=\left\{\varphi \mid \varphi: \Omega \times[t, T] \rightarrow \mathbb{R}^{d}\right.$ is an $\left(\mathcal{F}_{t}\right)_{t \geq 0}$-predictable process, and $\left.\mathbb{E}\left[\int_{t}^{T}\left|\varphi_{t}\right|^{2} d t\right]<+\infty\right\} ;$

$-\left[L^{2}(\mathcal{P} \otimes \mathcal{L})\right]^{k-1}=\left\{H \mid H=(H(1), \cdots, H(k-1)): \Omega \times[t, T] \times \mathbf{L} \rightarrow \mathbb{R}^{k-1}\right.$ is $\mathcal{P} \otimes \mathcal{L}$-measurable ${ }^{1}$ and $\left.\|H\|_{\left[L^{2}(\mathcal{P} \otimes \mathcal{L})\right]^{k-1}}=\left(\mathbb{E}\left[\int_{t}^{T} \sum_{l=1}^{k-1} H_{s}^{2}(l) \mathrm{d} s\right]\right)^{\frac{1}{2}}<+\infty\right\} ;$

- $\left[L^{2}(\mathbf{L} ; \mathbb{R})\right]^{k-1}=\{H(\cdot) \mid H(\cdot): \mathbf{L} \rightarrow \mathbb{R}\}$. Moreover, for a map $H(\cdot): \mathbf{L} \rightarrow \mathbb{R}$ we introduce the norm $\|$ $H \|_{\left[L^{2}(\mathbf{L} ; \mathbb{R})\right]^{k-1}}=\left[\sum_{l=1}^{k-1} H^{2}(l)\right]^{\frac{1}{2}}$.

We set $\mathcal{B}^{2}[t, T]=\mathcal{S}^{2}(t, T ; \mathbb{R}) \times \mathcal{S}^{2}(t, T ; \mathbb{R}) \times\left[L^{2}(\mathcal{P} \otimes \mathcal{L})\right]^{k-1} \times \mathcal{M}^{2}\left(t, T ; \mathbb{R}^{d}\right)$.

\subsection{BSDEs with jumps}

We consider the following BSDE with jumps:

$$
\left\{\begin{array}{l}
\mathrm{d} Y_{t}=-g\left(t, Y_{t}, H_{t}, Z_{t}\right) \mathrm{d} s+Z_{t} \mathrm{~d} B_{t}+\sum_{l=1}^{k-1} H_{t}(l) d \tilde{N}_{t}(l), t \in[0, T] \\
Y_{T}=\xi
\end{array}\right.
$$

where $T>0$ is an arbitrary but fixed time horizon, and the coefficient $g: \Omega \times[0, T] \times \mathbb{R} \times\left[L^{2}(\mathbf{L} ; \mathbb{R})\right]^{k-1} \times \mathbb{R}^{d} \rightarrow \mathbb{R}$ is $\mathcal{P}$-measurable, for every fixed $(y, h, z) \in \mathbb{R} \times\left[L^{2}(\mathbf{L} ; \mathbb{R})\right]^{k-1} \times \mathbb{R}^{d}$, and satisfies:

(H1) (i) There exists a constant $C \geq 0$ such that, $P$-a.s., for all $t \in[0, T], y_{1}, y_{2} \in \mathbb{R}, z_{1}, z_{2} \in \mathbb{R}^{d}, h_{1}, h_{2} \in$ $\left[L^{2}(\mathbf{L} ; \mathbb{R})\right]^{k-1}$,

$$
\left|g\left(t, y_{1}, h_{1}, z_{1}\right)-g\left(t, y_{2}, h_{2}, z_{2}\right)\right| \leq C\left(\left|y_{1}-y_{2}\right|+\left\|h_{1}-h_{2}\right\|_{\left[L^{2}(\mathbf{L} ; \mathbb{R})\right]^{k-1}}+\left|z_{1}-z_{2}\right|\right) ;
$$

\footnotetext{
${ }^{1} \mathcal{P}$ denotes the $\sigma$-field of $\left(\mathcal{F}_{t}\right)_{t \geq 0}$-predictable subsets of $\Omega \times[t, T]$.
} 
(ii) $\mathbb{E}\left[\left(\int_{0}^{T}|g(s, 0,0,0)| \mathrm{d} s\right)^{2}\right]<+\infty$.

Let us recall some well-known results.

Lemma 2.1. Under the condition $(\mathbf{H 1})$, for any random variable $\xi \in L^{2}\left(\Omega, \mathcal{F}_{T}, P ; \mathbb{R}\right)$, the BSDE with jumps (2.1) has a unique solution

$$
\left(Y_{t}, H_{t}, Z_{t}\right)_{t \in[0, T]} \in \mathcal{S}^{2}(0, T ; \mathbb{R}) \times\left[L^{2}(\mathcal{P} \otimes \mathcal{L})\right]^{k-1} \times \mathcal{M}^{2}\left(0, T ; \mathbb{R}^{d}\right)
$$

For some $g: \Omega \times[0, T] \times \mathbb{R} \times\left[L^{2}(\mathbf{L} ; \mathbb{R})\right]^{k-1} \times \mathbb{R}^{d} \rightarrow \mathbb{R}$ satisfying $(\mathbf{H 1})$, and for $i \in\{1,2\}$, suppose the drivers $g_{i}$ are of the form

$$
g_{i}\left(s, Y_{s}^{i}, H_{s}^{i}, Z_{s}^{i}\right)=g\left(s, Y_{s}^{i}, H_{s}^{i}, Z_{s}^{i}\right)+\varphi_{i}(s), d s d P-a . e .
$$

Denote by $\left(Y^{1}, H^{1}, Z^{1}\right),\left(Y^{2}, H^{2}, Z^{2}\right)$ the solution of the BSDE with jumps with the data $\left(\xi_{1}, g_{1}\right)$ and $\left(\xi_{2}, g_{2}\right)$, respectively. We have the following classical estimate:

Lemma 2.2. Under (H1), the difference of $\left(Y^{1}, H^{1}, Z^{1}\right),\left(Y^{2}, H^{2}, Z^{2}\right)$ satisfies:

$$
\begin{aligned}
& \left|Y_{t}^{1}-Y_{t}^{2}\right|^{2}+\frac{1}{2} \mathbb{E}\left[\int_{t}^{T} e^{\beta(s-t)}\left(\left|Y_{s}^{1}-Y_{s}^{2}\right|^{2}+\left|Z_{s}^{1}-Z_{s}^{2}\right|^{2}+\lambda \sum_{l=1}^{k-1}\left|H_{s}^{1}(l)-H_{s}^{2}(l)\right|^{2}\right) \mathrm{d} s \mid \mathcal{F}_{t}\right] \\
& \leq \mathbb{E}\left[e^{\beta(T-t)}\left|\xi_{1}-\xi_{2}\right|^{2} \mid \mathcal{F}_{t}\right]+\mathbb{E}\left[\int_{t}^{T} e^{\beta(s-t)}\left|\varphi_{1}(s)-\varphi_{2}(s)\right|^{2} \mathrm{~d} s \mid \mathcal{F}_{t}\right], \quad P \text {-a.s., } t \in[0, T],
\end{aligned}
$$

where $\beta \geq 2+2 C+4 C^{2}$.

For the details on the above results, please refer to Lemma 2.3 in Li, Wei [7]; see also Barles, Buckdahn and Pardoux [1].

\section{Stochastic Differential GAmes}

\subsection{Formulation}

First we introduce the definitions of the sets of admissible control processes. Define

$$
\begin{aligned}
& \mathcal{U}:=\left\{u:[0, T] \times \Omega \rightarrow U \text { is }\left(\mathcal{F}_{t}\right)_{t \geq 0} \text {-progressively measurable process }\right\}, \\
& \mathcal{V}:=\left\{v:[0, T] \times \Omega \rightarrow V \text { is }\left(\mathcal{F}_{t}\right)_{t \geq 0} \text {-progressively measurable process }\right\},
\end{aligned}
$$

where the control state spaces $U$ and $V$ are supposed to be compact metric spaces. $\mathcal{U}$ (resp., $\mathcal{V})$ is the set of admissible control processes for player I (resp., II).

For every $i \in \mathbf{K}$, we consider continuous maps

$$
\begin{aligned}
& b_{i}:[0, T] \times \mathbb{R} \times \mathbb{R} \times \mathbb{R}^{d} \times U \times V \rightarrow \mathbb{R}, \quad \sigma_{i}:[0, T] \times \mathbb{R} \times \mathbb{R} \times U \times V \rightarrow \mathbb{R}^{d}, \\
& f_{i}:[0, T] \times \mathbb{R} \times \mathbb{R}^{k} \times \mathbb{R}^{d} \times U \times V \rightarrow \mathbb{R}, \quad g_{i}: \mathbb{R} \rightarrow \mathbb{R} .
\end{aligned}
$$


Here we adopt the definition $\tilde{f}_{p}$ as $[2,8]$. For every $p \in \mathbf{K}$, we define $\tilde{f}_{p}:[0, T] \times \mathbb{R} \times \mathbb{R} \times \mathbb{R}^{k-1} \times \mathbb{R}^{d} \times U \times V \rightarrow \mathbb{R}$ by

$$
\tilde{f}_{p}(t, x, y, h, z, u, v)=f_{p}\left(t, x, a^{p}, z, u, v\right),
$$

where $h=(h(1), \ldots, h(k-1))$, and $a^{p}=\left(a_{1}^{p}, \ldots, a_{k}^{p}\right)$ are related by

$$
\left\{\begin{array}{lll}
a_{j}^{p}=y+h(k-p+j), & & j<p, \\
a_{p}^{p}=y, & & j=p, \\
a_{j}^{p}=y+h(j-p), & & j>p .
\end{array}\right.
$$

The vector $a^{p}=\left(a_{1}^{p}, \cdots, a_{k}^{p}\right)$ given by the right-hand side of $(3.1)$ will be denoted by $a^{p}[y, h]$. It is easy to check that, for all $(t, x) \in[0, T] \times \mathbb{R}, z \in \mathbb{R}^{d}, u \in U, v \in V$,

$$
\left\{\begin{array}{r}
f_{1}(t, x, a, z, u, v)=\tilde{f}_{1}\left(t, x, a_{1}, h^{1}, z, u, v\right), \\
f_{2}(t, x, a, z, u, v)=\tilde{f}_{2}\left(t, x, a_{2}, h^{2}, z, u, v\right), \\
\cdots \cdots \\
f_{k}(t, x, a, z, u, v)=\tilde{f}_{k}\left(t, x, a_{k}, h^{k}, z, u, v\right),
\end{array}\right.
$$

where $h^{p}=\left(h^{p}(1), \ldots, h^{p}(k-1)\right)$ with

$$
h^{p}(j)=a_{(p+j) \bmod (k)}-a_{p}= \begin{cases}a_{p+j}-a_{p}, & 1 \leq j \leq k-p, \\ a_{p+j-k}-a_{p}, & k-p+1 \leq j \leq k-1 .\end{cases}
$$

We assume that for every $i \in \mathbf{K}, b_{i}, \sigma_{i}, f_{i}, g_{i}$ satisfy the following conditions:

(B1) $b_{i}, \sigma_{i}, g_{i}$ are Lipschitz continuous in $(x, y, z)$, uniformly with respect to $(t, u, v)$; $f_{i}$ is Lipschitz continuous in $(x, a, z)$, uniformly with respect to $(t, u, v)$.

(B2) For all $t \in[0, T], u \in U, v \in V,(x, y, z),\left(x^{\prime}, y^{\prime}, z^{\prime}\right) \in \mathbb{R} \times \mathbb{R} \times \mathbb{R}^{d}, h, h^{\prime} \in\left[L^{2}(\mathbf{L} ; \mathbb{R})\right]^{k-1}$,

$$
\begin{array}{ll}
\text { (i) } \quad & \left(b_{i}(t, x, y, z, u, v)-b_{i}\left(t, x^{\prime}, y^{\prime}, z^{\prime}, u, v\right)\right)\left(y-y^{\prime}\right)+\left(\sigma_{i}(t, x, y, u, v)-\sigma_{i}\left(t, x^{\prime}, y^{\prime}, u, v\right)\right)\left(z-z^{\prime}\right) \\
& -\left(f_{i}\left(t, x, a^{i}[y, h], z, u, v\right)-f_{i}\left(t, x^{\prime}, a^{i}\left[y^{\prime}, h^{\prime}\right], z^{\prime}, u, v\right)\right)\left(x-x^{\prime}\right)+\lambda \sum_{l=1}^{k-1}\left(h(l)-h^{\prime}(l)\right)\left(x-x^{\prime}\right) \\
& \leqslant-\beta_{1}\left|x-x^{\prime}\right|^{2}-\beta_{2}\left|y-y^{\prime}\right|^{2}-\beta_{3}\left|z-z^{\prime}\right|^{2}-\beta_{4} \sum_{l=1}^{k-1}\left|h(l)-h^{\prime}(l)\right|^{2}, \\
\text { (ii) } \quad & \left(g_{i}(x)-g_{i}\left(x^{\prime}\right)\right)\left(x-x^{\prime}\right) \geq \mu_{1}\left|x-x^{\prime}\right|^{2},
\end{array}
$$

where the relationships between $h, y$ and $a^{i}[y, h]$, are presented in (3.1), similar to $h^{\prime}, y^{\prime}$ and $a^{i}\left[y^{\prime}, h^{\prime}\right]$; and $\mu_{1}, \beta_{1}, \beta_{2}, \beta_{3}, \beta_{4}$ are nonnegative constants with $\beta_{1}+\beta_{2}>0, \beta_{1}+\beta_{3}>0, \beta_{1}+\beta_{4}>0, \beta_{2}+\mu_{1}>$ $0, \beta_{3}+\mu_{1}>0$, and $\beta_{4}+\mu_{1}>0$.

(B3) For fixed $\lambda>0$, there exists a constant $K_{1} \geq 0$ such that for all $i, j \in \mathbf{K}, j \neq i,(t, x, z) \in[0, T] \times \mathbb{R} \times \mathbb{R}^{d}$, and $u \in U, v \in V, a, a^{\prime} \in \mathbb{R}^{k}$ with $a_{p}=a_{p}^{\prime}, \forall p \neq j$, and $a_{j} \geq a_{j}^{\prime}$,

$$
f_{i}(t, x, a, z, u, v)-f_{i}\left(t, x, a^{\prime}, z, u, v\right) \geq K_{1}\left(a_{j}-a_{j}^{\prime}\right) .
$$


For given admissible controls $u \in \mathcal{U}, v \in \mathcal{V}, i \in \mathbf{K}$, and initial data $(t, \eta) \in[0, T] \times L^{2}\left(\Omega, \mathcal{F}_{t}, P ; \mathbb{R}\right)$, let us consider the dynamics of our stochastic coupled controlled system

$$
\left\{\begin{aligned}
\mathrm{d} X_{s}^{t, \eta, i ; u, v}= & b_{N_{s}^{t, i}}\left(s, X_{s}^{t, \eta, i ; u, v}, Y_{s}^{t, \eta, i ; u, v}, Z_{s}^{t, \eta, i ; u, v}, u_{s}, v_{s}\right) \mathrm{d} s \\
& +\sigma_{N_{s}^{t, i}}\left(s, X_{s}^{t, \eta, i ; u, v}, Y_{s}^{t, \eta, i ; u, v}, u_{s}, v_{s}\right) \mathrm{d} B_{s}, \quad s \in[t, T], \\
X_{t}^{t, \eta, i ; u, v}= & \eta, \\
\mathrm{d} Y_{s}^{t, \eta, i ; u, v}= & -\tilde{f}_{N_{s}^{t, i}}\left(s, X_{s}^{t, \eta, i ; u, v}, Y_{s}^{t, \eta, i ; u, v}, H_{s}^{t, \eta, i ; u, v}, Z_{s}^{t, \eta, i ; u, v}, u_{s}, v_{s}\right) \mathrm{d} s \\
& +\lambda \sum_{l=1}^{k-1} H_{s}^{t, \eta, i ; u, v}(l) \mathrm{d} s+Z_{s}^{t, \eta, i ; u, v} \mathrm{~d} B_{s}+\sum_{l=1}^{k-1} H_{s}^{t, \eta, i ; u, v}(l) \mathrm{d} \tilde{N}_{s}(l), \\
Y_{T}^{t, \eta, i ; u, v}= & g_{N_{T}^{t, i}}\left(X_{T}^{t, \eta, i ; u, v}\right) .
\end{aligned}\right.
$$

Remark 3.1. Note that, the involved coefficient in BSDE (3.2) is $\tilde{f}_{p}$, not $f_{p}$. From the definition of $\tilde{f}_{p}$ and assumptions (B1)-(B3), it is easy to check that for every $i \in \mathbf{K}$,

(i) $\tilde{f}_{i}$ is Lipschitz with respect to $(x, y, h, z)$, uniformly with respect to $(t, u, v)$;

(ii) For all $t \in[0, T], u \in U, v \in V,(x, y, h, z),\left(x^{\prime}, y^{\prime}, h^{\prime}, z^{\prime}\right) \in \mathbb{R} \times \mathbb{R} \times\left[L^{2}(\mathbf{L} ; \mathbb{R})\right]^{k-1} \times \mathbb{R}^{d}$,

$$
\begin{aligned}
& \left(b_{i}(t, x, y, z, u, v)-b_{i}\left(t, x^{\prime}, y^{\prime}, z^{\prime}, u, v\right)\right)\left(y-y^{\prime}\right)+\left(\sigma_{i}(t, x, y, u, v)-\sigma_{i}\left(t, x^{\prime}, y^{\prime}, u, v\right)\right)\left(z-z^{\prime}\right) \\
& -\left(\tilde{f}_{i}(t, x, y, h, z, u, v)-\tilde{f}_{i}\left(t, x^{\prime}, y^{\prime}, h^{\prime}, z^{\prime}, u, v\right)\right)\left(x-x^{\prime}\right)+\lambda \sum_{l=1}^{k-1}\left(h(l)-h^{\prime}(l)\right)\left(x-x^{\prime}\right) \\
& \leqslant-\beta_{1}\left|x-x^{\prime}\right|^{2}-\beta_{2}\left|y-y^{\prime}\right|^{2}-\beta_{3}\left|z-z^{\prime}\right|^{2}-\beta_{4} \sum_{l=1}^{k-1}\left|h(l)-h^{\prime}(l)\right|^{2}
\end{aligned}
$$

where $\beta_{1}, \beta_{2}, \beta_{3}, \beta_{4}$ are the same constants as in (B2).

(iii) (B3) is equivalent with the condition that, for any $i, j \in \mathbf{K}, j \neq i, t \in[0, T],(x, y, z) \in \mathbb{R} \times \mathbb{R} \times \mathbb{R}^{d}$, and $u \in U, v \in V, h, h^{\prime} \in \mathbb{R}^{k-1}$ with $h_{p}=h_{p}^{\prime}, \forall p \neq j$, and $h_{j} \geq h_{j}^{\prime}$,

$$
\tilde{f}_{i}(t, x, y, h, z, u, v)-\tilde{f}_{i}\left(t, x, y, h^{\prime}, z, u, v\right) \geq K_{1}\left(h_{j}-h_{j}^{\prime}\right) .
$$

Remark 3.2. The authors of [2] used the assumption $K_{1}>-1$. However, if $K_{1}<0$, examples show that the comparison theorem does, in general, not hold, while for $K_{1} \geq 0$ the comparison result follows from the comparison theorem in [11] and a passage to the limit. We give more details in the Appendix A.1.

Remark 3.3. In fact, FBSDE (3.2) is a special case of fully coupled FBSDEs with jumps considered in $[6,7]$. Thus it follows from Lemma 2.1 in [6] that for every $u \in \mathcal{U}, v \in \mathcal{V},(3.2)$ has a unique solution $\left(X^{t, \eta, i ; u, v}, Y^{t, \eta, i ; u, v}, H^{t, \eta, i ; u, v}, Z^{t, \eta, i ; u, v}\right) \in \mathcal{B}^{2}[t, T]$ under the assumptions (B1) and (B2). Moreover, standard estimates for coupled FBSDEs with jumps (see, Prop. 3.1 in [6]) show that, there exists some constant $C>0$ such that, for all $t \in[0, T], i \in \mathbf{K}, u \in \mathcal{U}, v \in \mathcal{V}$ and $\eta, \eta^{\prime} \in L^{2}\left(\Omega, \mathcal{F}_{t}, P ; \mathbb{R}\right)$,

(i)

$$
\begin{aligned}
& \mathbb{E}\left[\sup _{t \leq s \leq T}\left|X_{s}^{t, \eta, i ; u, v}-X_{s}^{t, \eta^{\prime}, i ; u, v}\right|^{2}+\sup _{t \leq s \leq T}\left|Y_{s}^{t, \eta, i ; u, v}-Y_{s}^{t, \eta^{\prime 2}, i ; u, v}\right|^{2}+\int_{t}^{T}\left|Z_{s}^{t, \eta, i ; u, v}-Z_{s}^{t, \eta^{\prime}, i ; u, v}\right|^{2} \mathrm{~d} s\right. \\
& \left.\quad+\lambda \int_{t}^{T} \sum_{l=1}^{k-1}\left|H_{s}^{t, \eta, i ; u, v}(l)-H_{s}^{t, \eta^{\prime}, i ; u, v}(l)\right|^{2} \mathrm{~d} s \mid \mathcal{F}_{t}\right] \leq C\left|\eta-\eta^{\prime}\right|^{2}
\end{aligned}
$$

(ii) $\mathbb{E}\left[\sup _{t \leq s \leq T}\left|X_{s}^{t, \eta, i ; u, v}\right|^{2}+\sup _{t \leq s \leq T}\left|Y_{s}^{t, \eta, i ; u, v}\right|^{2}+\int_{t}^{T}\left|Z_{s}^{t, \eta, i ; u, v}\right|^{2} \mathrm{~d} s+\lambda \int_{t}^{T} \sum_{l=1}^{k-1}\left|H_{s}^{t, \eta, i ; u, v}(l)\right|^{2} \mathrm{~d} s \mid \mathcal{F}_{t}\right]$ $\leq C\left(1+|\eta|^{2}\right)$. 
Given control processes $u \in \mathcal{U}, v \in \mathcal{V}$, and the initial data $(t, x, i) \in[0, T] \times \mathbb{R} \times \mathbf{K}$, the cost functional of our stochastic differential game is defined as

$$
J(t, x, i ; u, v):=Y_{t}^{t, x, i ; u, v},
$$

where the process $Y^{t, x, i ; u, v}$ is the first component of the solution of FBSDE (3.2) with the initial condition $(t, x, i)$. The relations (3.3) and (3.4) imply that, there exists a constant $C>0$ such that for any $x, x^{\prime} \in \mathbb{R}$,

$$
\text { (ii) } \quad|J(t, x, i ; u, v)| \leq C(1+|x|) \text {, for all } i \in \mathbf{K}, u \in \mathcal{U}, v \in \mathcal{V} \text {. }
$$

As a consequence, for all $i \in \mathbf{K}, t \in[0, T], \eta \in L^{2}\left(\Omega, \mathcal{F}_{t}, P ; \mathbb{R}\right)$, we also have

$$
J(t, \eta, i ; u, v)=\left.J(t, x, i ; u, v)\right|_{x=\eta}=Y_{t}^{t, \eta, i ; u, v}, \quad \text { P-a.s. },
$$

which is a classical result in stochastic control theory, see, for example, Theorem 3.1 in [6].

Now we introduce the following subspaces of admissible controls. Let $\tau_{1}, \tau_{2}$ be two stopping times such that $t \leq \tau_{1}<\tau_{2} \leq T, P$-a.s. We define the random interval $\left[\left[\tau_{1}, \tau_{2}\right]\right]:=\left\{(t, \omega) \in[0, T] \times \Omega, \tau_{1}(\omega) \leq t \leq \tau_{2}(\omega)\right\}$.

Definition 3.4. Let $u^{0} \in U, v^{0} \in V$. A process $u=\left\{u_{r}(\omega),(r, \omega) \in\left[\left[\tau_{1}, \tau_{2}\right]\right]\right\} \quad\left(\operatorname{resp} ., v=\left\{v_{r}(\omega),(r, \omega) \in\right.\right.$ $\left.\left.\left[\left[\tau_{1}, \tau_{2}\right]\right]\right\}\right)$ is an admissible control for player I (resp., II) on $\left[\left[\tau_{1}, \tau_{2}\right]\right]$, if $u I_{\left[\left[\tau_{1}, \tau_{2}\right]\right]}+u^{0} I_{\left.[[0, T]] \backslash\left[\tau_{1}, \tau_{2}\right]\right]}$ (resp. $\left.v I_{\left[\left[\tau_{1}, \tau_{2}\right]\right]}+v^{0} I_{\left.[[0, T]] \backslash\left[\tau_{1}, \tau_{2}\right]\right]}\right)$ is $\left(\mathcal{F}_{r}\right)$-progressively measurable and with values in $U$ (resp., $V$ ). The set of all admissible controls for player I (resp., II) on $\left[\left[\tau_{1}, \tau_{2}\right]\right]$ is denoted by $\mathcal{U}_{\tau_{1}, \tau_{2}}$ (resp., $\mathcal{V}_{\tau_{1}, \tau_{2}}$ ). We identify two processes $u$ and $\bar{u}$ in $\mathcal{U}_{\tau_{1}, \tau_{2}}$ and write $u \equiv \bar{u}$ on $\left[\left[\tau_{1}, \tau_{2}\right]\right]$, if $P\left\{u=\bar{u}\right.$ a.e. in $\left.\left[\left[\tau_{1}, \tau_{2}\right]\right]\right\}=1$. Similarly we interpret $v=\bar{v}$ on $\left[\left[\tau_{1}, \tau_{2}\right]\right]$ in $\mathcal{V}_{\tau_{1}, \tau_{2}}$.

The nonanticipative strategies for the game are defined as follows:

Definition 3.5. A nonanticipative strategy for player I on $\left[\left[\tau_{1}, \tau_{2}\right]\right]$ is a mapping $\alpha: \mathcal{V}_{\tau_{1}, \tau_{2}} \rightarrow \mathcal{U}_{\tau_{1}, \tau_{2}}$ such that, for all $\mathbb{F}$-stopping time $S: \Omega \rightarrow\left[\left[\tau_{1}, \tau_{2}\right]\right]$ and all $v_{1}, v_{2} \in \mathcal{V}_{\tau_{1}, \tau_{2}}$, with $v_{1} \equiv v_{2}$ on $\left[\left[\tau_{1}, S\right]\right]$, it holds that $\alpha\left(v_{1}\right) \equiv \alpha\left(v_{2}\right)$ on $\left[\left[\tau_{1}, S\right]\right]$. Nonanticipative strategies for player II on $\left[\left[\tau_{1}, \tau_{2}\right]\right], \beta: \mathcal{U}_{\tau_{1}, \tau_{2}} \rightarrow \mathcal{V}_{\tau_{1}, \tau_{2}}$, are defined similarly. The set of all nonanticipative strategies $\alpha: \mathcal{V}_{\tau_{1}, \tau_{2}} \rightarrow \mathcal{U}_{\tau_{1}, \tau_{2}}$ for player I on $\left[\left[\tau_{1}, \tau_{2}\right]\right]$ is denoted by $\mathcal{A}_{\tau_{1}, \tau_{2}}$, while the set of all nonanticipative strategies $\beta: \mathcal{U}_{\tau_{1}, \tau_{2}} \rightarrow \mathcal{V}_{\tau_{1}, \tau_{2}}$ for player II on $\left[\left[\tau_{1}, \tau_{2}\right]\right]$ is denoted by $\mathcal{B}_{\tau_{1}, \tau_{2}}$.

The lower and upper value functions of our stochastic differential game are defined respectively as follows:

$$
\begin{aligned}
& \text { (lower) } \quad W_{i}(t, x):=\underset{\beta \in \mathcal{B}_{t, T}}{\operatorname{essinf}} \underset{u \in \mathcal{U}_{t, T}}{\operatorname{essup}} J(t, x, i ; u, \beta(u)), \\
& \text { (upper) } \quad U_{i}(t, x):=\operatorname{esssup}_{\alpha \in \mathcal{A}_{t, T}}^{\operatorname{essinf}} \underset{v \in \mathcal{V}_{t, T}}{\operatorname{ess}} J(t, x, i ; \alpha(v), v), \quad(t, x) \in[0, T] \times \mathbb{R}, i \in \mathbf{K} .
\end{aligned}
$$

Now we intend to establish the relationship between FBSDEs (3.2) and PDEs (1.1) via the lower value function $W_{i}, i \in \mathbf{K}$. In the last section, we will explain which system of HJBI equations the upper value function $U_{i}, i \in \mathbf{K}$ will be related with. As the essential infimum and the essential supremum of the $\mathcal{F}_{t}$-measurable cost functional $J(t, x, i ; u, \beta(u))$ over a family of control processes and strategies, $W_{i}(t, x)$ is an $\mathcal{F}_{t}$-measurable random variable. But it turns out to be deterministic. From now on, we only present the properties of the lower value function, the upper value function $U_{i}$ can be analyzed in the same manner.

Proposition 3.6. For any $(t, x, i) \in[0, T] \times \mathbb{R} \times \mathbf{K}$, the lower value function $W_{i}(t, x)$ is a deterministic function: $\mathbb{E}\left[W_{i}(t, x)\right]=W_{i}(t, x), P$-a.s.

Proof. This result is a direct consequence of Proposition 3.1 in Li and Wei [7]; see also Buckdahn, Hu and Li $[3]$. 
From the definition of the lower value function combined with (3.5), we see that the following results hold true.

Lemma 3.7. There exists a constant $C>0$ such that, for all $i \in \mathbf{K}, x, x^{\prime} \in \mathbb{R}, t \in[0, T]$,

(i) $\quad\left|W_{i}(t, x)-W_{i}\left(t, x^{\prime}\right)\right| \leq C\left|x-x^{\prime}\right|$;

(ii) $\quad\left|W_{i}(t, x)\right| \leq C(1+|x|)$.

Lemma 3.8. Suppose that (B1) and (B2) hold true. Then, for all $u \in \mathcal{U}, v \in \mathcal{V}$, the cost functional $J(t, x, i ; u, v)$ is monotonic in the following sense: for every $i \in \mathbf{K}, x, \bar{x} \in \mathbb{R}, t \in[0, T]$,

$$
(J(t, x, i ; u, v)-J(t, \bar{x}, i ; u, v))(x-\bar{x}) \geq 0, P \text {-a.s. }
$$

Furthermore, the lower value function $W_{i}(t, x)$ is monotonic: $\left(W_{i}(t, x)-W_{i}(t, \bar{x})\right)(x-\bar{x}) \geq 0, i \in \mathbf{K}, t \in$ $[0, T], x, \bar{x} \in \mathbb{R}$.

For the proof, please refer to Lemma 3.4 in $\mathrm{Li}$ and Wei [7].

\subsection{Dynamic Programming Principle}

To get the dynamic programming principle (DPP, for short), we adapt the notion of the stochastic backward semigroup which was first introduced by Peng [10] to our problem. Given $(t, x, i) \in[0, T] \times \mathbb{R} \times \mathbf{K}, u \in \mathcal{U}, v \in \mathcal{V}$, for all stopping times $\varsigma, \tau$ such that $t \leq \varsigma \leq \tau \leq T, P$-a.s., and $\xi \in L^{2}\left(\Omega, \mathcal{F}_{\tau}, P ; \mathbb{R}\right)$, we define

$$
G_{\varsigma, \tau}^{t, x, i ; u, v}[\xi]:=\hat{Y}_{\varsigma}^{t, x, i ; u, v}
$$

where $\left(\hat{X}_{s}^{t, x, i ; u, v}, \hat{Y}_{s}^{t, x, i ; u, v}, \hat{H}_{s}^{t, x, i ; u, v}, \hat{Z}_{s}^{t, x, i ; u, v}\right)_{s \in[t, \tau]}$ is the solution of the following FBSDE with the random time horizon $\tau$ :

$$
\left\{\begin{aligned}
\mathrm{d} \hat{X}_{s}^{t, x, i ; u, v}= & b_{N_{s}^{t, i}}\left(s, \hat{X}_{s}^{t, x, i ; u, v}, \hat{Y}_{s}^{t, x, i ; u, v}, \hat{Z}_{s}^{t, x, i ; u, v}, u_{s}, v_{s}\right) \mathrm{d} s \\
& +\sigma_{N_{s}^{t, i}}\left(s, \hat{X}_{s}^{t, x, i ; u, v}, \hat{Y}_{s}^{t, x, i ; u, v}, u_{s}, v_{s}\right) \mathrm{d} B_{s}, \\
\hat{X}_{t}^{t, x, i ; u, v}= & x, \\
\mathrm{~d} \hat{Y}_{s}^{t, x, i ; u, v}= & -\tilde{f}_{N_{s}^{t, i}}\left(s, \hat{X}_{s}^{t, x, i ; u, v}, \hat{Y}_{s}^{t, x, i ; u, v}, \hat{H}_{s}^{t, x, i ; u, v}, \hat{Z}_{s}^{t, x, i ; u, v}, u_{s}, v_{s}\right) \mathrm{d} s \\
& +\lambda \sum_{l=1}^{k-1} \hat{H}_{s}^{t, x, i ; u, v}(l) \mathrm{d} s+\hat{Z}_{s}^{t, x, i ; u, v} \mathrm{~d} B_{s}+\sum_{l=1}^{k-1} \hat{H}_{s}^{t, x, i ; u, v}(l) d \tilde{N}_{s}(l), \quad s \in[t, \tau], \\
\hat{Y}_{\tau}^{t, x, i ; u, v}= & \xi .
\end{aligned}\right.
$$

Remark 3.9. (i) By Lemma 2.5 in [7] equation (3.10) has a unique solution $\left(\hat{X}_{s}^{t, x, i ; u, v}, \hat{Y}_{s}^{t, x, i ; u, v}, \hat{H}_{s}^{t, x, i ; u, v}\right.$, $\left.\hat{Z}_{s}^{t, x, i ; u, v}\right)_{s \in[t, \tau]}$ under the assumptions (B1) and (B2)-(i).

(ii) When the terminal $\hat{Y}_{\tau}^{t, x, i ; u, v}$ also depends on the solution of the forward SDE, i.e., $\hat{Y}_{\tau}^{t, x, i ; u, v}=\Psi(\tau$, $\left.\hat{X}_{\tau}^{t, x, i ; u, v}\right)$, where $\Psi: \Omega \times \mathbb{R} \rightarrow \mathbb{R}$ is $\mathcal{F}_{\tau} \otimes \mathcal{B}(\mathbb{R})$-measurable, linear growth and Lipschitz in $x$, then, by Theorem 3.2 in [6], there exists some $\delta_{0}>0$ independent of $(t, x)$ and $(u, v)$, such that for any $0 \leq \delta \leq \delta_{0}$, (3.10) has a unique solution on the small interval $[t, t+\delta]$. Then we will consider $t \leq \varsigma \leq \tau \leq t+\delta$, and $\xi=\Psi\left(\tau, \hat{X}_{\tau}^{t, x, i ; u, v}\right)$, and have

$$
\hat{Y}_{\varsigma}^{t, x, i ; u, v}=G_{\varsigma, \tau}^{t, x, i ; u, v}\left[\Psi\left(\tau, \hat{X}_{\tau}^{t, x, i ; u, v}\right)\right],
$$

where $\hat{Y}_{s}^{t, x, i ; u, v}$ is the first component of the solution of (3.10) with the terminal $\Psi\left(\tau, \hat{X}_{\tau}^{t, x, i ; u, v}\right)$ at the random time horizon $\tau$. 
For the solution $\left(X^{t, x, i ; u, v}, Y^{t, x, i ; u, v}, H^{t, x, i ; u, v}, Z^{t, x, i ; u, v}\right)$ of FBSDEs (3.2) with $\eta=x$, it is easy to check that

$$
G_{t, \tau}^{t, x, i ; u, v}\left[Y_{\tau}^{t, x, i ; u, v}\right]=G_{t, T}^{t, x, i ; u, v}\left[g_{N_{T}^{t, i}}\left(X_{T}^{t, x, i ; u, v}\right)\right]=Y_{t}^{t, x, i ; u, v}=J(t, x, i ; u, v) .
$$

With the help of the notion of the backward semigroup, we have the following classical DPP which is the weak one in our paper.

Theorem 3.10. (Weak-DPP) Suppose (B1), (B2) and (B3). There exists some $\delta_{0}>0$ small enough such that, for any $0 \leq \delta \leq \delta_{0}, t \in[0, T-\delta], x \in \mathbb{R}, i \in \mathbf{K}$, we have

$$
W_{i}(t, x)=\operatorname{essinf}_{\beta \in \mathcal{B}_{t, t+\delta}} \operatorname{esssup}_{u \in \mathcal{U}_{t, t+\delta}} G_{t, t+\delta}^{t, x, i ; u, \beta(u)}\left[W_{N_{t+\delta}^{t, i}}\left(t+\delta, \tilde{X}_{t+\delta}^{t, x, i ; u, \beta(u)}\right)\right],
$$

where $\left(\tilde{X}^{t, x, i ; u, v}, \tilde{Y}^{t, x, i ; u, v}, \tilde{Z}^{t, x, i ; u, v}, \tilde{H}^{t, x, i ; u, v}\right)$ is the solution of the following coupled FBSDE with jumps on $[t, t+\delta]:$

$$
\left\{\begin{aligned}
\mathrm{d} \tilde{X}_{s}^{t, x, i ; u, v}= & b_{N_{s}^{t, i}}\left(s, \tilde{X}_{s}^{t, x, i ; u, v}, \tilde{Y}_{s}^{t, x, i ; u, v}, \tilde{Z}_{s}^{t, x, i ; u, v}, u_{s}, v_{s}\right) \mathrm{d} s \\
& +\sigma_{N_{s}^{t, i}}\left(s, \tilde{X}_{s}^{t, x, i ; u, v}, \tilde{Y}_{s}^{t, x, i ; u, v}, u_{s}, v_{s}\right) \mathrm{d} B_{s}, \quad s \in[t, t+\delta], \\
\tilde{X}_{t}^{t, x, i ; u, v}= & x, \\
\mathrm{~d} \tilde{Y}_{s}^{t, x, i ; u, v}= & -\tilde{f}_{N_{s}^{t, i}}\left(s, \tilde{X}_{s}^{t, x, i ; u, v}, \tilde{Y}_{s}^{t, x, i ; u, v}, \tilde{H}_{s}^{t, x, i ; u, v}, \tilde{Z}_{s}^{t, x, i ; u, v}, u_{s}, v_{s}\right) \mathrm{d} s \\
& +\lambda \sum_{l=1}^{k-1} \tilde{H}_{s}^{t, x, i ; u, v}(l) \mathrm{d} s+\tilde{Z}_{s}^{t, x, i ; u, v} \mathrm{~d} B_{s}+\sum_{l=1}^{k-1} \tilde{H}_{s}^{t, x, i ; u, v}(l) d \tilde{N}_{s}(l), \\
\tilde{Y}_{t+\delta}^{t, x, i ; u, v}= & W_{N_{t+\delta}^{t, i}}\left(t+\delta, \tilde{X}_{t+\delta}^{t, x, i ; u, v}\right) .
\end{aligned}\right.
$$

Its proof uses the same arguments as those in the proof of Theorem 3.1 in [7] and therefore is omitted. We will provide the proof of the strong DPP, which generalizes Theorem 3.10.

Remark 3.11. From Lemma 3.8 it follows that, for all $i \in \mathbf{K}, t \in[0, T], x, \bar{x} \in \mathbb{R},\left(W_{i}(t, x)-W_{i}(t, \bar{x})\right)(x-\bar{x}) \geq$ 0 , which doesn't mean (B2)-(ii). However, Lemma 3.7 tells us that, for all $i \in \mathbf{K}, t \in[0, T], W_{i}(t, x)$ is Lipschitz in $x$. Therefore, from Remark 3.9-(ii), the stochastic backward semigroup in (3.12) makes sense.

Next we recall the following continuity property of the lower value function $W_{i}(t, x)$ in $t$, which is also a consequence of Theorem 3.2 in Li, Wei [7].

Proposition 3.12. Assume (B1), (B2) and (B3). Then, there exists a constant $C$ such that, for every $x \in$ $\mathbb{R}, i \in \mathbf{K}, t, t^{\prime} \in[0, T]$,

$$
\left|W_{i}(t, x)-W_{i}\left(t^{\prime}, x\right)\right| \leq C(1+|x|)\left|t-t^{\prime}\right|^{\frac{1}{2}} .
$$

That means, $W_{i}(t, x)$ is $\frac{1}{2}$-Hölder continuous in $t$.

To prepare the strong DPP, we need the following auxiliary results.

Lemma 3.13. For any stopping time $\tau: t \leq \tau \leq T$, we have

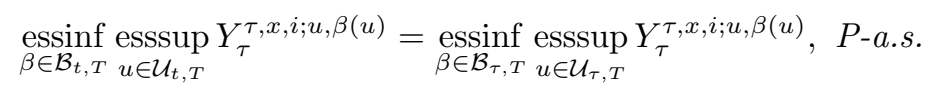

Proof. Here, we briefly present the details for (3.14) in two steps. 
(i) The first one is to prove

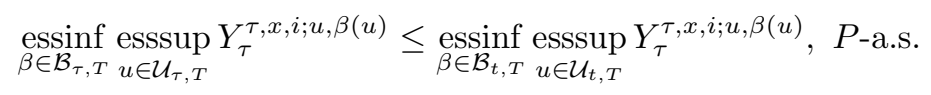

For any arbitrarily fixed $\beta \in \mathcal{B}_{t, T}$, given any $u_{0} \in \mathcal{U}_{t, \tau}$, we define the restriction $\beta_{1}^{u_{0}}$ of $\beta$ to $\mathcal{B}_{\tau, T}$ :

$$
\beta_{1}^{u_{0}}\left(u_{1}\right)=\left.\beta\left(u_{0} \oplus u_{1}\right)\right|_{[\tau, T]}, u_{1} \in \mathcal{U}_{\tau, T},
$$

where $u:=u_{0} \oplus u_{1}=u_{0} I_{[t, \tau)}+u_{1} I_{[\tau, T]} \in \mathcal{U}_{t, T}$. It is easy to check that, according to Definition 3.5, $\beta_{1}^{u_{0}} \in \mathcal{B}_{\tau, T}$.

Note that $Y_{\tau}^{\tau, x, i ; u, \beta(u)}$ denotes the state of the solution $Y$ of $(3.2)$ with initial data $(\tau, x, i)$ at $\tau$. Combining this with the definitions of $u$ and $\beta_{1}^{u_{0}}$, we get

$$
Y_{\tau}^{\tau, x, i ; u_{1}, \beta_{1}^{u_{0}}\left(u_{1}\right)}=Y_{\tau}^{\tau, x, i ; u, \beta(u)} .
$$

Thus, for all $\beta \in \mathcal{B}_{t, T}$,

$$
\underset{\beta^{\prime} \in \mathcal{B}_{\tau, T}}{\operatorname{essinf}} \operatorname{esssup}_{u_{1} \in \mathcal{U}_{\tau, T}} Y_{\tau}^{\tau, x, i ; u_{1}, \beta^{\prime}\left(u_{1}\right)} \leq \operatorname{esssup}_{u_{1} \in \mathcal{U}_{\tau, T}} Y_{\tau}^{\tau, x, i ; u_{1}, \beta_{1}^{u_{0}}\left(u_{1}\right)}=\operatorname{esssup}_{u_{1} \in \mathcal{U}_{\tau, T}} Y_{\tau}^{\tau, x, i ; u_{0} \oplus u_{1}, \beta\left(u_{0} \oplus u_{1}\right)} \leq \operatorname{esssup}_{u \in \mathcal{U}_{t, T}} Y_{\tau}^{\tau, x, i ; u, \beta(u)},
$$

which yields

$$
\underset{\beta^{\prime} \in \mathcal{B}_{\tau, T}}{\operatorname{essinf}} \operatorname{esssup}_{u_{1} \in \mathcal{U}_{\tau, T}} Y_{\tau}^{\tau, x, i ; u_{1}, \beta^{\prime}\left(u_{1}\right)} \leq \underset{\beta \in \mathcal{B}_{t, T}}{\operatorname{essinf}} \operatorname{esssup}_{u \in \mathcal{U}_{t, T}} Y_{\tau}^{\tau, x, i ; u, \beta(u)} .
$$

Hence, (3.15) holds true.

(ii) We prove now the converse relation

$$
\underset{\beta \in \mathcal{B}_{\tau, T}}{\operatorname{essinf}} \underset{u \in \mathcal{U}_{\tau, T}}{\operatorname{esssup}} Y_{\tau}^{\tau, x, i ; u, \beta(u)} \geq \underset{\beta \in \mathcal{B}_{t, T}}{\operatorname{essinf}} \operatorname{esssup}{ }_{u \in \mathcal{U}_{t, T}} Y_{\tau}^{\tau, x, i ; u, \beta(u)}, P \text {-a.s. }
$$

For any $\varepsilon>0$, there exists a $\beta_{1}^{\varepsilon} \in \mathcal{B}_{\tau, T}$ such that

$$
\underset{\beta_{1} \in \mathcal{B}_{\tau, T}}{\operatorname{essinf}} \underset{u_{1} \in \mathcal{U}_{\tau, T}}{\operatorname{esssup}} Y_{\tau}^{\tau, x, i ; u_{1}, \beta_{1}\left(u_{1}\right)} \geq \underset{u_{1} \in \mathcal{U}_{\tau, T}}{\operatorname{esssup}} Y_{\tau}^{\tau, x, i ; u_{1}, \beta_{1}^{\varepsilon}\left(u_{1}\right)}-\varepsilon, \text { P-a.s. }
$$

for the proof it is standard now, see, for instance, Remark 3.7 in [7].

Given an arbitrary $\beta_{0} \in \mathcal{B}_{t, \tau}$, for any $u \in \mathcal{U}_{t, T}$, define $u_{1}$ as the restriction of $u$ to $[\tau, T]$, i.e., $u_{1}=\left.u\right|_{[\tau, T]}$, and $\beta^{\varepsilon}$ as the extension of $\beta_{1}^{\varepsilon}$ to $[t, T]: \beta^{\varepsilon}(u):=\beta_{0}\left(\left.u\right|_{[t, \tau]}\right) \oplus \beta_{1}^{\varepsilon}\left(u_{1}\right)$. Obviously, $\beta^{\varepsilon} \in \mathcal{B}_{t, T}$. Similar to (3.16), we also have $Y_{\tau}^{\tau, x, i, u_{1}, \beta_{1}^{\varepsilon}\left(u_{1}\right)}=Y_{\tau}^{\tau, x, i ; u, \beta^{\varepsilon}(u)}$. Therefore,

$$
\begin{aligned}
& \underset{\beta_{1} \in \mathcal{B}_{\tau, T}}{\operatorname{essinf}} \operatorname{esssup} Y_{1} \in \mathcal{U}_{\tau, T}^{\tau, x} \\
& =\operatorname{esssup}_{\tau \in \mathcal{U}_{t, T}} Y_{\tau}^{\tau, x, i ; u, u_{1}, \beta_{1}\left(u_{1}\right)} \geq \underset{u_{1} \in \mathcal{U}_{\tau, T}}{\operatorname{esssup}}-\varepsilon \geq \underset{\beta \in \mathcal{B}_{t, T}}{\operatorname{essinf}} Y_{u \in \mathcal{U}_{t, T}}^{\tau, x, i ; u_{1}, \beta_{1}^{\varepsilon}\left(u_{1}\right)}-\varepsilon \\
& \operatorname{esssup}_{\tau}^{\tau, x, i ; u, \beta(u)}-\varepsilon, \text { P-a.s. }
\end{aligned}
$$

From the arbitrariness of $\varepsilon$, we get (3.17).

Combining the above two steps, we complete the proof. 
For any $t \in[0, T]$, let $\tau$ be a stopping time such that $t \leq \tau \leq T$. For any positive integer $N$, let $t_{j}^{N}=t+\frac{(T-t) j}{N}$, $0 \leq j \leq N, t_{-1}^{N}:=0$. We define

$$
\tau_{N}:=\sum_{j=0}^{N} t_{j}^{N} I_{\left\{t_{j-1}^{N}<\tau \leq t_{j}^{N}\right\}}
$$

then $\tau_{N}$ is $\sigma\{\tau\}$-measurable stopping time, and $\tau_{N} \downarrow \tau$, as $N \uparrow \infty$.

Lemma 3.14. For the stopping time $\tau_{N}$ in $(3.18)$ and $\eta \in L^{2}\left(\Omega, \mathcal{F}_{\tau}, P ; \mathbb{R}\right)$, we have

$$
W_{i}\left(\tau_{N}, \eta\right)=\underset{\beta \in \mathcal{B}_{t, T}}{\operatorname{essinf}} \operatorname{esssup} \mathbb{u \in \mathcal { U } _ { t , T }}\left[Y_{\tau_{N}}^{\tau_{N}, \eta, i ; u, \beta(u)} \mid \mathcal{F}_{\tau}\right], \text { P-a.s. }
$$

Proof. For $\eta \in L^{2}\left(\Omega, \mathcal{F}_{\tau}, P ; \mathbb{R}\right),(u, v) \in \mathcal{U}_{t, T} \times \mathcal{V}_{t, T}$, we know $\left(X^{\tau, \eta, i ; u, v}, Y^{\tau, \eta, i ; u, v}, H^{\tau, \eta, i ; u, v}, Z^{\tau, \eta, i ; u, v}\right)$ is the unique solution of FBSDE (3.2) with $\tau$ instead of $t$.

From the uniqueness of solution $\left(X^{\tau_{N}, x, i ; u, v}, Y^{\tau_{N}, x, i ; u, v}, H^{\tau_{N}, x, i ; u, v}, Z^{\tau_{N}, x, i ; u, v}\right)$ and the fact that also $\sum_{j=0}^{N}$ $\left(X^{t_{j}^{N}, x, i ; u, v}, Y^{t_{j}^{N}, x, i ; u, v}, H^{t_{j}^{N}, x, i ; u, v}, Z^{t_{j}^{N}, x, i ; u, v}\right) I_{\left\{\tau_{N}=t_{j}^{N}\right\}}$ solves FBSDE (3.2) with $\tau_{N}$ instead of $t$, it follows that

$$
\left(X^{\tau_{N}, x, i ; u, v}, Y^{\tau_{N}, x, i ; u, v}, H^{\tau_{N}, x, i ; u, v}, Z^{\tau_{N}, x, i ; u, v}\right)=\sum_{j=0}^{N}\left(X^{t_{j}^{N}, x, i ; u, v}, Y^{t_{j}^{N}, x, i ; u, v}, H^{t_{j}^{N}, x, i ; u, v}, Z^{t_{j}^{N}, x, i ; u, v}\right) I_{\left\{\tau_{N}=t_{j}^{N}\right\}},
$$

and in particular,

$$
Y_{\tau_{N}, x, i ; u, v}^{\tau_{\tau^{N}}}=\sum_{j=0}^{N} Y_{t_{j}^{N}}^{t_{j}^{N}, x, i ; u, v} I_{\left\{\tau_{N}=t_{j}^{N}\right\}}=\sum_{j=0}^{N} J\left(t_{j}^{N}, x, i ; u, v\right) I_{\left\{\tau_{N}=t_{j}^{N}\right\}}, \text { P-a.s. }
$$

Hence,

$$
\begin{aligned}
& W_{i}\left(\tau_{N}, x\right)=\sum_{j=0}^{N} W_{i}\left(t_{j}^{N}, x\right) I_{\left\{\tau_{N}=t_{j}^{N}\right\}}=\sum_{j=0}^{N} I_{\left\{\tau_{N}=t_{j}^{N}\right\}} \underset{\beta \in \mathcal{B}_{t_{j}^{N}, T}}{\operatorname{essinf}} \underset{u \in \mathcal{U}_{t_{j}^{N}, T}}{\operatorname{esssup}} J\left(t_{j}^{N}, x, i ; u, \beta(u)\right) \\
& =\underset{\beta \in \mathcal{B}_{t, T}}{\operatorname{essinf}} \underset{u \in \mathcal{U}_{t, T}}{\operatorname{essup}} Y_{\tau_{N}}^{\tau_{N}, x, i ; u, \beta(u)}, \text { P-a.s., }
\end{aligned}
$$

where the latter equality follows from standard arguments for the essential infimum and the essential supermum of families of $\mathcal{F}_{\tau_{N}}$-measurable random variables as well as Lemma 3.13.

Again, from the above relation and from standard arguments (see, for example, Rem. 3.7 in [7]) it follows that for fixed $i$ :

(i) For any $\varepsilon>0$, any $\beta \in \mathcal{B}_{t, T}$, there exists some $u^{\varepsilon} \in \mathcal{U}_{t, T}$, such that

$$
W_{i}\left(\tau_{N}, x\right) \leq Y_{\tau_{N}}^{\tau_{N}, x, i ; u^{\varepsilon}, \beta\left(u^{\varepsilon}\right)}+\varepsilon, \text { P-a.s. }
$$

(ii) For any $\varepsilon>0$, there exists some $\beta^{\varepsilon} \in \mathcal{B}_{t, T}$, such that for all $u \in \mathcal{U}_{t, T}$,

$$
W_{i}\left(\tau_{N}, x\right) \geq Y_{\tau_{N}}^{\tau_{N}, x, i ; u, \beta^{\varepsilon}(u)}-\varepsilon, \text { P-a.s. }
$$


As $W_{i}\left(\tau_{N}, x\right)$ is $\mathcal{F}_{\tau}$-measurable ( $W_{i}$ is continuous, and hence Borel measurable, and $\tau_{N}$ is $\sigma\{\tau\}$-measurable), we have

(i) For any $\varepsilon>0$, and any $\beta \in \mathcal{B}_{t, T}$, there exists some $u^{\varepsilon} \in \mathcal{U}_{t, T}$, such that

$$
W_{i}\left(\tau_{N}, x\right) \leq \mathbb{E}\left[Y_{\tau_{N}}^{\tau_{N}, x, i ; u^{\varepsilon}, \beta\left(u^{\varepsilon}\right)} \mid \mathcal{F}_{\tau}\right]+\varepsilon, \text { P-a.s. }
$$

(ii) For any $\varepsilon>0$, there exists some $\beta^{\varepsilon} \in \mathcal{B}_{t, T}$, such that for all $u \in \mathcal{U}_{t, T}$,

$$
W_{i}\left(\tau_{N}, x\right) \geq \mathbb{E}\left[Y_{\tau_{N}}^{\tau_{N}, x, i ; u, \beta^{\varepsilon}(u)} \mid \mathcal{F}_{\tau}\right]-\varepsilon, \text { P-a.s. }
$$

Hence,

$$
W_{i}\left(\tau_{N}, x\right)=\underset{\beta \in \mathcal{B}_{t, T}}{\operatorname{essinf}} \operatorname{esssup} \underset{u \in \mathcal{U}_{t, T}}{ } \mathbb{E}\left[Y_{\tau_{N}}^{\tau_{N}, x, i ; u, \beta(u)} \mid \mathcal{F}_{\tau}\right] \text {, P-a.s. }
$$

Finally, (3.5) and (3.8) combined with a standard approximation argument for $\eta \in L^{2}\left(\Omega, \mathcal{F}_{\tau}, P ; \mathbb{R}\right)$ allow to show

$$
W_{i}\left(\tau_{N}, \eta\right)=\left.W_{i}\left(\tau_{N}, x\right)\right|_{x=\eta}=\underset{\beta \in \mathcal{B}_{t, T}}{\operatorname{essinf}} \operatorname{esssup}_{u \in \mathcal{U}_{t, T}} \mathbb{E}\left[Y_{\tau_{N}}^{\tau_{N}, \eta, i ; u, \beta(u)} \mid \mathcal{F}_{\tau}\right], \text { P-a.s. }
$$

Next, we have the following estimate.

Lemma 3.15. For all stopping time $\tau(t \leq \tau \leq T), \eta \in L^{2}\left(\Omega, \mathcal{F}_{\tau}, P ; \mathbb{R}\right), i \in \mathbf{K},(u, v) \in \mathcal{U}_{t, T} \times \mathcal{V}_{t, T}$, we have

$$
\left|\mathbb{E}\left[Y_{\tau_{N}}^{\tau_{N}, \eta, i ; u, v} \mid \mathcal{F}_{\tau}\right]-Y_{\tau}^{\tau, \eta, i ; u, v}\right|^{2} \leq C\left(\tau_{N}-\tau\right)\left(1+|\eta|^{2}\right), \quad P \text {-a.s., } \quad N \geq 1
$$

Proof. First of all, we have

$$
\left|\mathbb{E}\left[Y_{\tau_{N}}^{\tau_{N}, \eta, i ; u, v} \mid \mathcal{F}_{\tau}\right]-Y_{\tau}^{\tau, \eta, i ; u, v}\right| \leq\left|\mathbb{E}\left[Y_{\tau_{N}}^{\tau_{N}, \eta, i ; u, v}-Y_{\tau_{N}}^{\tau, \eta, i ; u, v} \mid \mathcal{F}_{\tau}\right]\right|+\left|\mathbb{E}\left[Y_{\tau_{N}}^{\tau, \eta, i ; u, v} \mid \mathcal{F}_{\tau}\right]-Y_{\tau}^{\tau, \eta, i ; u, v}\right| .
$$

Now we work on the two terms at the right side of the above inequality. To simplify the notations, we omit the indication of the controls $u, v$ in the following proof. For the forward equation, from (3.3)-(ii) (extended to stopping times), we get

$$
\begin{aligned}
& \mathbb{E}\left[\left|\eta-X_{\tau_{N}, \eta, i}\right|^{2} \mid \mathcal{F}_{\tau}\right] \\
= & \mathbb{E}\left[\left|\int_{\tau}^{\tau_{N}} b_{N_{s}^{\tau, i}}\left(s, X_{s}^{\tau, \eta, i}, Y_{s}^{\tau, \eta, i}, Z_{s}^{\tau, \eta, i}\right) \mathrm{d} s+\int_{\tau}^{\tau_{N}} \sigma_{N_{s}^{\tau, i}}\left(s, X_{s}^{\tau, \eta, i}, Y_{s}^{\tau, \eta, i}\right) \mathrm{d} B_{s}\right|^{2} \mid \mathcal{F}_{\tau}\right] \\
\leq & C\left(1+|\eta|^{2}\right)\left|\tau_{N}-\tau\right| .
\end{aligned}
$$

For the backward equations with the different data $(\tau, \eta, i)$ and $\left(\tau_{N}, \eta, i\right)$, we apply Lemma 2.2 by setting

$$
\begin{aligned}
& \xi_{1}:=g_{N_{T}^{\tau, i}}\left(X_{T}^{\tau, \eta, i}\right), \quad \xi_{2}:=g_{N_{T}^{\tau_{N}, i}}\left(X_{T}^{\tau_{N}, \eta, i}\right), \quad \varphi_{1}(s):=0, \\
& \varphi_{2}(s):=\tilde{f}_{N_{s}^{\tau_{N}, i}}\left(s, X_{s}^{\tau_{N}, \eta, i}, Y_{s}^{\tau_{N}, \eta, i}, H_{s}^{\tau_{N}, \eta, i}, Z_{s}^{\tau_{N}, \eta, i}\right)-\tilde{f}_{N_{s}^{\tau, i}}\left(s, X_{s}^{\tau, \eta, i}, Y_{s}^{\tau_{N}, \eta, i}, H_{s}^{\tau_{N}, \eta, i}, Z_{s}^{\tau_{N}, \eta, i}\right), s \in\left[\tau_{N}, T\right],
\end{aligned}
$$


then we get

$$
\begin{aligned}
& \mathbb{E}\left[\left|Y_{\tau_{N}}^{\tau_{N}, \eta, i}-Y_{\tau_{N}}^{\tau, \eta, i}\right|^{2} \mid \mathcal{F}_{\tau}\right] \\
\leq & C \mathbb{E}\left[\left|g_{N_{T}^{\tau_{N}, i}}\left(X_{T}^{\tau_{N}, \eta, i}\right)-g_{N_{T}^{\tau, i}}\left(X_{T}^{\tau, \eta, i}\right)\right|^{2} \mid \mathcal{F}_{\tau}\right]+C \mathbb{E}\left[\int_{\tau_{N}}^{T}\left|\varphi_{2}(s)\right|^{2} d s \mid \mathcal{F}_{\tau}\right] \\
\leq & C \mathbb{E}\left[\left|g_{N_{T}^{\tau_{N}, i}}\left(X_{T}^{\tau_{N}, \eta, i}\right)-g_{N_{T}^{\tau, i}}\left(X_{T}^{\tau, \eta, i}\right)\right|^{2} \mid \mathcal{F}_{\tau}\right] \\
& +C \mathbb{E}\left[\int_{\tau_{N}}^{T}\left|X_{s}^{\tau_{N}, \eta, i}-X_{s}^{\tau, \eta, i}\right|^{2} d s \mid \mathcal{F}_{\tau}\right]+C \mathbb{E}\left[\int_{\tau_{N}}^{T}\left|I_{f}(s)\right|^{2} d s \mid \mathcal{F}_{\tau}\right],
\end{aligned}
$$

where $I_{f}(s)=\tilde{f}_{N_{s}^{\tau_{N}, i}}\left(s, X_{s}^{\tau_{N}, \eta, i}, Y_{s}^{\tau_{N}, \eta, i}, H_{s}^{\tau_{N}, \eta, i}, Z_{s}^{\tau_{N}, \eta, i}\right)-\tilde{f}_{N_{s}^{\tau, i}}\left(s, X_{s}^{\tau_{N}, \eta, i}, Y_{s}^{\tau_{N}, \eta, i}, H_{s}^{\tau_{N}, \eta, i}, Z_{s}^{\tau_{N}, \eta, i}\right)$.

Firstly, we consider $\mathbb{E}\left[\left|g_{N_{T}^{\tau_{N}, i}}\left(X_{T}^{\tau_{N}, \eta, i}\right)-g_{N_{T}^{\tau, i}}\left(X_{T}^{\tau, \eta, i}\right)\right|^{2} \mid \mathcal{F}_{\tau}\right]$. Obviously,

$$
\begin{aligned}
& \mathbb{E}\left[\left|g_{N_{T}^{\tau_{N}, i}}\left(X_{T}^{\tau_{N}, \eta, i}\right)-g_{N_{T}^{\tau, i}}\left(X_{T}^{\tau, \eta, i}\right)\right|^{2} \mid \mathcal{F}_{\tau}\right] \\
\leq & C \mathbb{E}\left[\mathbf{1}_{\left\{N_{\tau_{N}}^{\tau, i}=i\right\}}\left|g_{N_{T}^{\tau_{N}, i}}\left(X_{T}^{\tau_{N}, \eta, i}\right)-g_{N_{T}^{\tau, i}}\left(X_{T}^{\tau, \eta, i}\right)\right|^{2} \mid \mathcal{F}_{\tau}\right] \\
& +C \mathbb{E}\left[\mathbf{1}_{\left\{N_{\tau_{N}}^{\tau, i} \neq i\right\}}\left|g_{N_{T}^{\tau_{N}, i}}\left(X_{T}^{\tau_{N}, \eta, i}\right)-g_{N_{T}^{\tau, i}}\left(X_{T}^{\tau_{N}, \eta, i}\right)\right|^{2} \mid \mathcal{F}_{\tau}\right] \\
& +C \mathbb{E}\left[\mathbf{1}_{\left\{N_{\tau_{N}}^{\tau, i} \neq i\right\}}\left|g_{N_{T}^{\tau, i}}\left(X_{T}^{\tau_{N}, \eta, i}\right)-g_{N_{T}^{\tau, i}}\left(X_{T}^{\tau, \eta, i}\right)\right|^{2} \mid \mathcal{F}_{\tau}\right]=I_{1}+I_{2}+I_{3},
\end{aligned}
$$

where

$$
\begin{gathered}
I_{1}=C \mathbb{E}\left[\mathbf{1}_{\left\{N_{\tau_{N}}^{\tau, i}=i\right\}}\left|g_{N_{T}^{\tau_{N}, i}}\left(X_{T}^{\tau_{N}, \eta, i}\right)-g_{N_{T}^{\tau, i}}\left(X_{T}^{\tau, \eta, i}\right)\right|^{2} \mid \mathcal{F}_{\tau}\right], \\
I_{2}=C \mathbb{E}\left[\mathbf{1}_{\left\{N_{\tau_{N}}^{\tau, i} \neq i\right\}}\left|g_{N_{T}^{\tau_{N}, i}}\left(X_{T}^{\tau_{N}, \eta, i}\right)-g_{N_{T}^{\tau, i}}\left(X_{T}^{\tau_{N}, \eta, i}\right)\right|^{2} \mid \mathcal{F}_{\tau}\right], \\
I_{3}=C \mathbb{E}\left[\mathbf{1}_{\left\{N_{\tau_{N}}^{\tau, i} \neq i\right\}}\left|g_{N_{T}^{\tau, i}}\left(X_{T}^{\tau_{N}, \eta, i}\right)-g_{N_{T}^{\tau, i}}\left(X_{T}^{\tau, \eta, i}\right)\right|^{2} \mid \mathcal{F}_{\tau}\right] .
\end{gathered}
$$

For $I_{1}$, on $\left\{N_{\tau_{N}}^{\tau, i}=i\right\}$, we know, $N_{T}^{\tau_{N}, i}=N_{T}^{\tau_{N}, N_{\tau_{N}}^{\tau, i}}=N_{T}^{\tau, i}$, and from the uniqueness of the solution we get $X_{T}^{\tau, \eta, i}=X_{T}^{\tau_{N}, X_{\tau}^{\tau, \eta, i}, N_{\tau_{N}}^{\tau, i}}=X_{T}^{\tau_{N}, X_{\tau_{N}}^{\tau, \eta, i}, i}, P$-a.s. Hence, from (3.3)-(i) and (3.20), we know

$$
\begin{aligned}
I_{1} & =C \mathbb{E}\left[\mathbf{1}_{\left\{N_{\tau_{N}}^{\tau, i}=i\right\}} \mathbb{E}\left[\left|g_{N_{T}^{\tau_{N}, i}}\left(X_{T}^{\tau_{N}, \eta, i}\right)-g_{N_{T}^{\tau, i}}\left(X_{T}^{\tau_{N}, X_{\tau_{N}}^{\tau, \eta, i}, i}\right)\right|^{2} \mid \mathcal{F}_{\tau_{N}}\right] \mid \mathcal{F}_{\tau}\right] \\
& \leq C \mathbb{E}\left[\mathbf{1}_{\left\{N_{\tau_{N}}^{\tau, i}=i\right\}}\left|\eta-X_{\tau_{N}, i, i}\right|^{2} \mid \mathcal{F}_{\tau}\right] \\
& \leq C\left(1+|\eta|^{2}\right)\left|\tau_{N}-\tau\right| .
\end{aligned}
$$


For $I_{2}$, from the linear growth property of $g_{i}, i \in \mathbf{K}$ and (3.3)-(ii),

$$
\begin{aligned}
I_{2} & =C \mathbb{E}\left[\mathbf{1}_{\left\{N_{\tau_{N}}^{\tau, i} \neq i\right\}} \mathbb{E}\left[\left|g_{N_{T}^{\tau_{N}, i}}\left(X_{T}^{\tau_{N}, \eta, i}\right)-g_{N_{T}^{\tau, i}}\left(X_{T}^{\tau_{N}, \eta, i}\right)\right|^{2} \mid \mathcal{F}_{\tau_{N}}\right] \mid \mathcal{F}_{\tau}\right] \\
& \leq C \mathbb{E}\left[\mathbf{1}_{\left\{N_{\tau_{N}}^{\tau, i} \neq i\right\}} \mathbb{E}\left[\left(1+\left|X_{T}^{\tau_{N}, \eta, i}\right|^{2}\right) \mid \mathcal{F}_{\tau_{N}}\right] \mid \mathcal{F}_{\tau}\right] \\
& \leq C \mathbb{E}\left[\mathbf{1}_{\left\{N_{\tau_{N}}^{\tau, i} \neq i\right\}} \mid \mathcal{F}_{\tau}\right]\left(1+|\eta|^{2}\right)=C P\left\{N_{\tau_{N}}^{\tau, i} \neq i\right\}\left(1+|\eta|^{2}\right) \\
& =C\left(1-e^{-\lambda(k-1)\left(\tau_{N}-\tau\right)}\right)\left(1+|\eta|^{2}\right) \leq C\left(1+|\eta|^{2}\right)\left|\tau_{N}-\tau\right| .
\end{aligned}
$$

For $I_{3}$, from the Lipschitz property of $g_{i}, i \in \mathbf{K},(3.3)-(i i)$, and (3.20),

$$
\begin{aligned}
& I_{3}=C \mathbb{E}\left[\mathbf{1}_{\left\{N_{\tau_{N}}^{\tau, i} \neq i\right\}} \mathbb{E}\left[\left|g_{N_{T}^{\tau, i}}\left(X_{T}^{\tau_{N}, \eta, i}\right)-g_{N_{T}^{\tau, i}}\left(X_{T}^{\tau, \eta, i}\right)\right|^{2} \mid \mathcal{F}_{\tau_{N}}\right] \mid \mathcal{F}_{\tau}\right] \\
& \leq C \mathbb{E}\left[\mathbf{1}_{\left\{N_{\tau_{N}}^{\tau, i} \neq i\right\}} \mathbb{E}\left[\left|X_{T}^{\tau_{N}, \eta, i}-X_{T}^{\tau_{N}, X_{\tau_{N}}^{\tau, \eta, i}, N_{\tau_{N}}^{\tau, i}}\right|^{2} \mid \mathcal{F}_{\tau_{N}}\right] \mid \mathcal{F}_{\tau}\right] \\
& \leq C \mathbb{E}\left[\mathbf{1}_{\left\{N_{\tau_{N}}^{\tau, i} \neq i\right\}}\left(1+|\eta|^{2}+\left|X_{\tau_{N}}^{\tau, \eta, i}\right|^{2}\right) \mid \mathcal{F}_{\tau}\right] \\
& \leq C\left(1+|\eta|^{2}\right)\left|\tau_{N}-\tau\right|+C \mathbb{E}\left[\mathbf{1}_{\left\{N_{\tau_{N}}^{\tau, i} \neq i\right\}}\left|X_{\tau_{N}}^{\tau, \eta, i}\right|^{2} \mid \mathcal{F}_{\tau}\right] \\
& \leq C\left(1+|\eta|^{2}\right)\left|\tau_{N}-\tau\right|+C \mathbb{E}\left[\mathbf{1}_{\left\{N_{\tau_{N}}^{\tau, i} \neq i\right\}}\left|X_{\tau_{N}}^{\tau, \eta, i}-\eta\right|^{2} \mid \mathcal{F}_{\tau}\right]+C \mathbb{E}\left[\mathbf{1}_{\left\{N_{\tau_{N}}^{\tau, i} \neq i\right\}}|\eta|^{2} \mid \mathcal{F}_{\tau}\right] \\
& \leq C\left(1+|\eta|^{2}\right)\left|\tau_{N}-\tau\right| \text {. }
\end{aligned}
$$

Therefore, we get

$$
\mathbb{E}\left[\left|g_{N_{T}^{\tau_{N}, i}}\left(X_{T}^{\tau_{N}, \eta, i}\right)-g_{N_{T}^{\tau, i}}\left(X_{T}^{\tau, \eta, i}\right)\right|^{2} \mid \mathcal{F}_{\tau}\right] \leq C\left(1+|\eta|^{2}\right)\left|\tau_{N}-\tau\right|
$$

Now we focus on $\mathbb{E}\left[\int_{\tau_{N}}^{T}\left|X_{s}^{\tau_{N}, \eta, i}-X_{s}^{\tau, \eta, i}\right|^{2} d s \mid \mathcal{F}_{\tau}\right]$. Similarly, from (3.3), (3.20) and the proof for $I_{3}$, we have

$$
\begin{aligned}
& \mathbb{E}\left[\int_{\tau_{N}}^{T}\left|X_{s}^{\tau_{N}, \eta, i}-X_{s}^{\tau, \eta, i}\right|^{2} d s \mid \mathcal{F}_{\tau}\right] \\
\leq & T \mathbb{E}\left[\sup _{s \in\left[\tau_{N}, T\right]}\left|X_{s}^{\tau_{N}, \eta, i}-X_{s}^{\tau, \eta, i}\right|^{2} \mid \mathcal{F}_{\tau}\right] \\
= & T \mathbb{E}\left[\mathbf{1}_{\left\{N_{\tau_{N}}^{\tau, i}=i\right\}} \mathbb{E}\left[\sup _{s \in\left[\tau_{N}, T\right]}\left|X_{s}^{\tau_{N}, \eta, i}-X_{s}^{\tau_{N}, X_{\tau_{N}}^{\tau, \eta, i}, i}\right|^{2} \mid \mathcal{F}_{\tau_{N}}\right] \mid \mathcal{F}_{\tau}\right] \\
& +T \mathbb{E}\left[\mathbf{1}_{\left\{N_{\tau_{N}}^{\tau, i} \neq i\right\}} \mathbb{E}\left[\sup _{s \in\left[\tau_{N}, T\right]}\left|X_{s}^{\tau_{N}, \eta, i}-X_{s}^{\tau_{N}, X_{\tau_{N}}^{\tau, \eta, i}, N_{\tau_{N}}^{\tau, i}}\right|^{2} \mid \mathcal{F}_{\tau_{N}}\right] \mid \mathcal{F}_{\tau}\right] \\
\leq & T \mathbb{E}\left[\mathbf{1}_{\left\{N_{\tau_{N}}^{\tau, i}=i\right\}}\left|X_{\tau_{N}}^{\tau, \eta, i}-\eta\right|^{2} \mid \mathcal{F}_{\tau}\right]+T \mathbb{E}\left[\mathbf{1}_{\left\{N_{\tau_{N}}^{\tau, i} \neq i\right\}}\left(1+|\eta|^{2}+\left|X_{\tau_{N}}^{\tau, \eta, i}\right|^{2}\right) \mid \mathcal{F}_{\tau}\right] \\
\leq & C\left(\tau_{N}-\tau\right)\left(1+|\eta|^{2}\right) .
\end{aligned}
$$


Combined with (3.21), (3.22) and (3.23), using (3.3)-(ii) and the proof for $I_{2}$, we have

$$
\begin{aligned}
& \mathbb{E}\left[\left|Y_{\tau_{N}}^{\tau_{N}, \eta, i}-Y_{\tau_{N}}^{\tau, \eta, i}\right|^{2} \mid \mathcal{F}_{\tau}\right] \\
\leq & C\left(1+|\eta|^{2}\right)\left|\tau_{N}-\tau\right|+C \mathbb{E}\left[\int_{\tau_{N}}^{T}\left|I_{f}(s)\right|^{2} d s \mid \mathcal{F}_{\tau}\right] \\
\leq & C\left(1+|\eta|^{2}\right)\left|\tau_{N}-\tau\right| \\
& +C \mathbb{E}\left[\mathbf{1}_{\left\{N_{\tau_{N}}^{\tau, i} \neq i\right\}} \mathbb{E}\left[\int_{\tau_{N}}^{T}\left(1+\left|X_{s}^{\tau_{N}, \eta, i}\right|^{2}+\left|Y_{s}^{\tau_{N}, \eta, i}\right|^{2}+\left|Z_{s}^{\tau_{N}, \eta, i}\right|^{2}+\sum_{l=1}^{k-1}\left|H_{s}^{\tau_{N}, \eta, i}(l)\right|^{2}\right) \mathrm{d} s \mid \mathcal{F}_{\tau_{N}}\right] \mid \mathcal{F}_{\tau}\right] \\
\leq & C\left(1+|\eta|^{2}\right)\left|\tau_{N}-\tau\right|+C\left(1+|\eta|^{2}\right) \mathbb{E}\left[\mathbf{1}_{\left\{N_{\tau_{N}}^{\tau, i} \neq i\right\}} \mid \mathcal{F}_{\tau}\right] \\
\leq & C\left(1+|\eta|^{2}\right)\left|\tau_{N}-\tau\right|,
\end{aligned}
$$

where we have used the linear growth properties of $\tilde{f}_{i}, i \in \mathbf{K}$.

On the other hand, as $\tau_{N} \geq \tau$ is $\sigma\{\tau\}$-measurable and still from (3.3)-(ii) we know

$$
\begin{aligned}
& \left|\mathbb{E}\left[Y_{\tau_{N}}^{\tau, \eta, i} \mid \mathcal{F}_{\tau}\right]-Y_{\tau}^{\tau, \eta, i}\right| \\
& =\left|\mathbb{E}\left[\int_{\tau}^{\tau_{N}} \tilde{f}_{N_{s}^{\tau, i}}\left(s, X_{s}^{\tau, \eta, i}, Y_{s}^{\tau, \eta, i}, H_{s}^{\tau, \eta, i}, Z_{s}^{\tau, \eta, i}\right) \mathrm{d} s-\int_{\tau}^{\tau_{N}} \lambda \sum_{l=1}^{k-1} H_{s}^{\tau, \eta, i}(l) \mathrm{d} s \mid \mathcal{F}_{\tau}\right]\right| \\
& \leq C\left(\tau_{N}-\tau\right)^{\frac{1}{2}}\left(\mathbb{E}\left[\int_{\tau}^{\tau_{N}}\left(1+\left|X_{s}^{\tau, \eta, i}\right|^{2}+\left|Y_{s}^{\tau, \eta, i}\right|^{2}+\sum_{l=1}^{k-1}\left|H_{s}^{\tau, \eta, i}(l)\right|^{2}+\left|Z_{s}^{\tau, \eta, i}\right|^{2}\right) \mathrm{d} s \mid \mathcal{F}_{\tau}\right]\right)^{\frac{1}{2}} \\
& \leq C\left(\tau_{N}-\tau\right)^{\frac{1}{2}}(1+|\eta|), \text { P-a.s. }
\end{aligned}
$$

Therefore, from (3.19), (3.24) and (3.25), we get

$$
\left|\mathbb{E}\left[Y_{\tau_{N}}^{\tau_{N}, \eta, i} \mid \mathcal{F}_{\tau}\right]-Y_{\tau}^{\tau, \eta, i}\right|^{2} \leq C\left(\tau_{N}-\tau\right)\left(1+|\eta|^{2}\right), \text { P-a.s., } N \geq 1
$$

Based on the above results, we generalize the results for the lower value function $W_{i}(t, x),(t, x) \in[0, T] \times \mathbb{R}$, $i \in \mathbf{K}$ to the case for stopping times $\tau$ and random variables $\eta \in L^{2}\left(\Omega, \mathcal{F}_{\tau}, P ; \mathbb{R}\right)$

Proposition 3.16. For every fixed initial data $(t, i) \in[0, T] \times \mathbf{K}$, every stopping time $\tau$ with values in $[t, T]$, and all $\eta \in L^{2}\left(\Omega, \mathcal{F}_{\tau}, P ; \mathbb{R}\right)$,

$$
W_{N_{\tau}^{t, i}}(\tau, \eta)=\operatorname{essinf}_{\beta \in \mathcal{B}_{\tau, T}} \operatorname{esssup}_{u \in \mathcal{U}_{\tau, T}} Y_{\tau}^{\tau, \eta, N_{\tau}^{t, i} ; u, \beta(u)}, \text { P-a.s. }
$$

Proof. As Lemma 3.15 implies that

$$
\begin{aligned}
& \left|\underset{\beta \in \mathcal{B}_{t, T}}{\operatorname{essinf}} \underset{u \in \mathcal{U}_{t, T}}{\operatorname{esssup}} \mathbb{E}\left[Y_{\tau_{N}}^{\tau_{N}, \eta, i ; u, \beta(u)} \mid \mathcal{F}_{\tau}\right]-\underset{\beta \in \mathcal{B}_{t, T}}{\operatorname{essinf}} \operatorname{esssup} Y_{u \in \mathcal{U}_{t, T}}^{\tau, \eta, i ; u, \beta(u)}\right|^{2} \\
& \leq C\left(\tau_{N}-\tau\right)\left(1+|\eta|^{2}\right) \leq C \frac{T-t}{N}\left(1+|\eta|^{2}\right) \rightarrow 0 \text {, as } N \rightarrow \infty \text {, P-a.s., }
\end{aligned}
$$


and $W_{i}$ is continuous, we get from Lemma 3.14 that

$$
W_{i}(\tau, \eta)=\underset{\beta \in \mathcal{B}_{t, T}}{\operatorname{essinf}} \operatorname{esssup}_{u \in \mathcal{U}_{t, T}} Y_{\tau}^{\tau, \eta, i ; u, \beta(u)}, \text { P-a.s., } i \in \mathbf{K} .
$$

As $\mathbf{K}$ is finite, and $N_{\tau}^{t, i}$ is $\mathcal{F}_{\tau}$-measurable, it follows that from Lemma 3.13

$$
W_{N_{\tau}^{t, i}}(\tau, \eta)=\operatorname{essinf}_{\beta \in \mathcal{B}_{t, T}} \operatorname{esssup}_{u \in \mathcal{U}_{t, T}} Y_{\tau}^{\tau, \eta, N_{\tau}^{t, i} ; u, \beta(u)}=\operatorname{essinf}_{\beta \in \mathcal{B}_{\tau, T}}^{\operatorname{esssup}} u \in \mathcal{U}_{\tau, T} Y_{\tau}^{\tau, \eta, N_{\tau}^{t, i} ; u, \beta(u)}, \text { P-a.s., } i \in \mathbf{K} .
$$

The following result is the strong DPP which plays a crucial role in the proof of the existence of a viscosity solution.

Theorem 3.17. (Strong-DPP) Suppose (B1), (B2) and (B3). There exists some $\delta_{0}>0$ small enough such that, for all $t \in\left[0, T-\delta_{0}\right]$, and all stopping time $\tau$ with $0 \leq t \leq \tau \leq t+\delta_{0} \leq T, x \in \mathbb{R}, i \in \mathbf{K}$, we have

$$
W_{i}(t, x)=\underset{\beta \in \mathcal{B}_{t, \tau}}{\operatorname{essinf}} \operatorname{esssup} G_{u \in \mathcal{U}_{t, \tau}}^{t, x, i ; u, \beta(u)}\left[W_{N_{\tau}^{t, i}}\left(\tau, \bar{X}_{\tau}^{t, x, i ; u, \beta(u)}\right)\right], \quad P \text {-a.s. },
$$

where $\left(\bar{X}^{t, x, i ; u, v}, \bar{Y}^{t, x, i ; u, v}, \bar{Z}^{t, x, i ; u, v}, \bar{H}^{t, x, i ; u, v}\right)$ is the solution of the following FBSDE with jumps on $[t, \tau]$ :

$$
\left\{\begin{aligned}
d \bar{X}_{s}^{t, x, i ; u, v}= & b_{N_{s}^{t, i}}\left(s, \bar{X}_{s}^{t, x, i ; u, v}, \bar{Y}_{s}^{t, x, i ; u, v}, \bar{Z}_{s}^{t, x, i ; u, v}, u_{s}, v_{s}\right) \mathrm{d} s \\
& +\sigma_{N_{s}^{t, i}}^{t}\left(s, \bar{X}_{s}^{t, x, i ; u, v}, \bar{Y}_{s}^{t, x, i ; u, v}, u_{s}, v_{s}\right) \mathrm{d} B_{s}, \quad s \in[t, \tau] \\
\bar{X}_{t}^{t, x, i ; u, v}= & x, \\
d \bar{Y}_{s}^{t, x, i ; u, v}= & -\tilde{f}_{N_{s}^{t, i}}\left(s, \bar{X}_{s}^{t, x, i ; u, v}, \bar{Y}_{s}^{t, x, i ; u, v}, \bar{H}_{s}^{t, x, i ; u, v}, \bar{Z}_{s}^{t, x, i ; u, v}, u_{s}, v_{s}\right) \mathrm{d} s \\
& +\lambda \sum_{l=1}^{k-1} \bar{H}_{s}^{t, x, i ; u, v}(l) \mathrm{d} s+\bar{Z}_{s}^{t, x, i ; u, v} \mathrm{~d} B_{s}+\sum_{l=1}^{k-1} \bar{H}_{s}^{t, x, i ; u, v}(l) d \tilde{N}_{s}(l) \\
\bar{Y}_{\tau}^{t, x, i ; u, v}= & W_{N_{\tau}^{t, i}}\left(\tau, \bar{X}_{\tau}^{t, x, i ; u, v}\right) .
\end{aligned}\right.
$$

We postpone the proof to the appendix.

\section{VisCOSITY SOLUTION}

\subsection{Existence}

In this subsection, we take advantage of the above results to prove that the value function $\mathbf{W}(t, x)=$ $\left(W_{1}(t, x), W_{2}(t, x), \cdots, W_{k}(t, x)\right),(t, x) \in[0, T] \times \mathbb{R}$, is a viscosity solution of the following system of coupled HJBI equations:

$$
\left\{\begin{array}{l}
\frac{\partial W_{i}}{\partial t}(t, x)+\sup _{u \in U} \inf _{v \in V}\left\{L_{u, v}^{i} W_{i}(t, x)+f_{i}\left(t, x, \mathbf{W}(t, x), D W_{i}(t, x) \sigma_{i}\left(t, x, W_{i}(t, x), u, v\right), u, v\right)\right\}=0 \\
W_{i}(T, x)=g_{i}(x), \quad(t, x, i) \in[0, T) \times \mathbb{R} \times \mathbf{K}
\end{array}\right.
$$

where for each $t \in[0, T), i \in \mathbf{K}, \varphi \in C^{2}([0, T] \times \mathbb{R})$, the differential operator is

$$
\begin{aligned}
L_{u, v}^{i} \varphi(t, x)= & \frac{1}{2} \operatorname{tr}\left(\sigma_{i} \sigma_{i}^{*}(t, x, \varphi(t, x), u, v) D^{2} \varphi(t, x)\right) \\
& +b_{i}\left(t, x, \varphi(t, x), D \varphi(t, x) \sigma_{i}(t, x, \varphi(t, x), u, v), u, v\right) D \varphi(t, x) .
\end{aligned}
$$

First we present the definition of viscosity solution for HJBI equation (4.1). 
Definition 4.1. Let $\mathbf{W}=\left(W_{1}, W_{2}, \cdots, W_{k}\right)$ belong to $C\left([0, T] \times \mathbb{R} ; \mathbb{R}^{k}\right)$. The function $\mathbf{W}$ is said to be (i) a viscosity subsolution of the system (4.1) if $W_{i}(T, x) \leq g_{i}(x), i \in \mathbf{K}, x \in \mathbb{R}$, and for all $i \in \mathbf{K}, \phi \in$ $C_{l, b}^{3}([0, T] \times \mathbb{R})$, for all $(t, x) \in[0, T) \times \mathbb{R}$ such that $(t, x)$ is a local maximum point of $W_{i}-\phi$, we have

$$
\frac{\partial \phi}{\partial t}(t, x)+\sup _{u \in U} \inf _{v \in V}\left\{L_{u, v}^{i} \phi(t, x)+f_{i}\left(t, x, \mathbf{W}(t, x), D \phi(t, x) \sigma_{i}\left(t, x, W_{i}(t, x), u, v\right), u, v\right)\right\} \geq 0
$$

(ii) a viscosity supersolution of the system (4.1) if $W_{i}(T, x) \geq g_{i}(x), i \in \mathbf{K}, x \in \mathbb{R}$, and for all $i \in \mathbf{K}, \phi \in$ $C_{l, b}^{3}([0, T] \times \mathbb{R})$, for all $(t, x) \in[0, T) \times \mathbb{R}$ such that $(t, x)$ is a local minimum point of $W_{i}-\phi$, we have

$$
\frac{\partial \phi}{\partial t}(t, x)+\sup _{u \in U} \inf _{v \in V}\left\{L_{u, v}^{i} \phi(t, x)+f_{i}\left(t, x, \mathbf{W}(t, x), D \phi(t, x) \sigma_{i}\left(t, x, W_{i}(t, x), u, v\right), u, v\right)\right\} \leq 0
$$

(iii) a viscosity solution of the system (4.1) if $\mathbf{W}$ is both a viscosity subsolution and a viscosity supersolution of (4.1).

Here $C_{l, b}^{3}([0, T] \times \mathbb{R})$ denotes the set of the real-valued functions that are continuously differentiable up to the third order and whose derivatives of order 1 to 3 are bounded.

The following is the main result.

Theorem 4.2. Assume that (B1)-(B3) hold. Moreover, suppose that $0<\lambda<\frac{1}{(k-1) T}$. Then the function $\mathbf{W}$ is a viscosity solution of the system (4.1) of coupled HJBI equations.

We begin with proving some auxiliary results first in order to prepare for the proof of Theorem 4.2. Let $(t, x, i) \in[0, T) \times \mathbb{R} \times \mathbf{K}$. For any given $\delta>0$ with $t+\delta \leq T$, the stopping time $\tau^{\delta}$ is defined as

$$
\tau^{\delta}=(t+\delta) \wedge \inf \left\{s \geq t: N_{s}^{t, i} \neq i\right\}
$$

We consider the following system:

$$
\left\{\begin{aligned}
\mathrm{d} X_{s}^{u, v}= & b_{i}\left(s, X_{s}^{u, v}, Y_{s}^{u, v}, Z_{s}^{u, v}, u_{s}, v_{s}\right) \mathrm{d} s+\sigma_{i}\left(s, X_{s}^{u, v}, Y_{s}^{u, v}, u_{s}, v_{s}\right) \mathrm{d} B_{s}, \quad s \in\left[t, \tau^{\delta}\right], \\
X_{t}^{u, v}= & x, \\
\mathrm{~d} Y_{s}^{u, v}= & -\tilde{f}_{i}\left(s, X_{s}^{u, v}, Y_{s}^{u, v}, H_{s}^{u, v}, Z_{s}^{u, v}, u_{s}, v_{s}\right) \mathrm{d} s+\lambda \sum_{l=1}^{k-1} H_{s}^{u, v}(l) \mathrm{d} s \\
& +Z_{s}^{u, v} \mathrm{~d} B_{s}+\sum_{l=1}^{k-1} H_{s}^{u, v}(l) \mathrm{d} \tilde{N}_{s}(l), \quad s \in\left[t, \tau^{\delta}\right], \\
Y_{\tau^{\delta}}^{u, v}= & \phi\left(\tau^{\delta}, X_{\tau^{\delta}}^{u, v}\right) \mathbf{1}_{\left\{N_{\tau^{\delta}}^{t, i}=i\right\}}+\sum_{l=1}^{k-1} W_{(l+i) \bmod (k)}\left(\tau^{\delta}, X_{\tau^{\delta}}^{u, v}\right) \mathbf{1}_{\left\{N_{\tau^{\delta}}^{t, i}=(l+i) \bmod (k)\right\}},
\end{aligned}\right.
$$

where $\phi \in C_{l, b}^{3}([0, T] \times \mathbb{R})$. From the wellposedness and regularity results of FBSDEs (see the Thms. 3.2 and 3.4 in [6]), under the assumption (B1), we know there exists some $\delta_{0}>0$ only depending on the Lipschitz constants of the coefficients, such that for all $\delta \in\left(0, \delta_{0}\right],(4.2)$ has a unique solution $\left(X^{u, v}, Y^{u, v}, H^{u, v}, Z^{u, v}\right) \in \mathcal{B}^{2}\left[t, \tau^{\delta}\right]$; 
and for all $p \geq 2$, there is some constant $C_{p}>0$ such that for all $\delta \in\left(0, \delta_{0}\right]$,

$$
\begin{array}{cc}
\text { (i) } \quad \mathbb{E}\left[\sup _{t \leq s \leq \tau^{\delta}}\left|X_{s}^{u, v}\right|^{p}+\sup _{t \leq s \leq \tau^{\delta}}\left|Y_{s}^{u, v}\right|^{p}+\left(\int_{t}^{\tau^{\delta}}\left|Z_{s}^{u, v}\right|^{2} \mathrm{~d} s\right)^{\frac{p}{2}}\right. \\
\left.\quad+\left(\lambda \int_{t}^{\tau^{\delta}} \sum_{l=1}^{k-1}\left|H_{s}^{u, v}(l)\right|^{2} \mathrm{~d} s\right)^{\frac{p}{2}} \mid \mathcal{F}_{t}\right] \leq C_{p}\left(1+|x|^{p}\right), \text { P-a.s.; } \\
\text { (ii) } \quad \mathbb{E}\left[\sup _{t \leq s \leq \tau^{\delta}}\left|X_{s}^{u, v}-x\right|^{p} \mid \mathcal{F}_{t}\right] \leq C_{p} \delta\left(1+|x|^{p}\right), \quad \text { P-a.s.; } \\
\text { (iii) } \quad \mathbb{E}\left[\left(\int_{t}^{\tau^{\delta}}\left|Z_{s}^{u, v}\right|^{2} \mathrm{~d} s\right)^{\frac{p}{2}}+\left(\lambda \int_{t}^{\tau^{\delta}} \sum_{l=1}^{k-1}\left|H_{s}^{u, v}(l)\right|^{2} \mathrm{~d} s\right)^{\frac{p}{2}} \mid \mathcal{F}_{t}\right] \leq C_{p} \delta^{\frac{p}{2}}\left(1+|x|^{p}\right), \quad \text { P-a.s. }
\end{array}
$$

Now we define

$$
\tilde{Y}_{s}^{1, i, u, v}:=\phi\left(s, X_{s}^{u, v}\right)+\sum_{l=1}^{k-1} \int_{t}^{s}\left(W_{(l+i) \bmod (k)}\left(r, X_{r}^{u, v}\right)-\phi\left(r, X_{r}^{u, v}\right)\right) d N_{r}(l), s \in\left[t, \tau^{\delta}\right] .
$$

Lemma 4.3. Let the process $\left(Y_{s}^{1, i, u, v}\right)_{s \in\left[t, \tau^{\delta}\right]}$ be defined as the first component of the solution $\left(Y^{1, i, u, v}, Z^{1, i, u, v}\right.$, $\left.H^{1, i, u, v}\right)$ of the following BSDE with jumps:

$$
\left\{\begin{aligned}
\mathrm{d} Y_{s}^{1, i, u, v}= & -F\left(s, X_{s}^{u, v}, Y_{s}^{1, i, u, v}, H_{s}^{1, i, u, v}, Z_{s}^{1, i, u, v}, u_{s}, v_{s}\right) \mathrm{d} s+\lambda \sum_{l=1}^{k-1} H_{s}^{1, i, u, v}(l) \mathrm{d} s \\
& +Z_{s}^{1, i, u, v} \mathrm{~d} B_{s}+\sum_{l=1}^{k-1} H_{s}^{1, i, u, v}(l) d \tilde{N}_{s}(l), \quad s \in\left[t, \tau^{\delta}\right], \\
Y_{\tau^{\delta}}^{1, i, u, v}= & 0,
\end{aligned}\right.
$$

where we have used the notations

$$
\begin{aligned}
& L(s, \tilde{x}, y, h, z, u, v) \\
& =\frac{\partial \phi}{\partial s}(s, \tilde{x})+\frac{1}{2} \operatorname{tr}\left(\sigma_{i} \sigma_{i}^{*}(s, \tilde{x}, y, u, v) D^{2} \phi(s, \tilde{x})\right)+b_{i}(s, \tilde{x}, y, z, u, v) D \phi(s, \tilde{x})+\tilde{f}_{i}(s, \tilde{x}, y, h, z, u, v), \\
& F(s, \tilde{x}, y, h, z, u, v) \\
& =L\left(s, \tilde{x}, y+\phi(s, \tilde{x}), h+\mathbf{W}_{(i+\cdot) \bmod (k)}(s, \tilde{x})-\phi(s, \tilde{x}) \mathbf{1}, z+D \phi(s, \tilde{x}) \sigma_{i}(s, \tilde{x}, y+\phi(s, \tilde{x}), u, v), u, v\right),
\end{aligned}
$$

and $h+\mathbf{W}_{(i+\cdot) \bmod (k)}(s, \tilde{x})-\phi(s, \tilde{x}) \mathbf{1}=\left(h_{j}+W_{(i+j) \bmod (k)}(s, \tilde{x})-\phi(s, \tilde{x})\right)_{1 \leq j \leq k-1}$. Then, for all $s \in[t, T]$,

$$
Y_{s \wedge \tau^{\delta}}^{1, i, u, v}=G_{s \wedge \tau^{\delta}, \tau^{\delta}}^{t, x, i, u, v}\left[\phi\left(\tau^{\delta}, X_{\tau^{\delta}}^{u, v}\right) 1_{\left\{N_{\tau^{\delta}}^{t, i}=i\right\}}+\sum_{l=1}^{k-1} W_{(l+i) \bmod (k)}\left(\tau^{\delta}, X_{\tau^{\delta}}^{u, v}\right) 1_{\left\{N_{\tau^{\delta}}^{t, i}=(l+i) \bmod (k)\right\}}\right]-\tilde{Y}_{s \wedge \tau^{\delta}}^{1, i, u}
$$

Remark 4.4. There exists some constant $C>0$ such that for all $(\tilde{x}, y, h, z),\left(\tilde{x}^{\prime}, y^{\prime}, h^{\prime}, z^{\prime}\right) \in \mathbb{R} \times \mathbb{R} \times \mathbb{R}^{k-1} \times \mathbb{R}^{d}$, for any $s \in[t, T], u \in U, v \in V$,

(i) $\left|L(s, \tilde{x}, y, h, z, u, v)-L\left(s, \tilde{x}^{\prime}, y^{\prime}, h^{\prime}, z^{\prime}, u, v\right)\right|$ $\leq C\left(1+|\tilde{x}|+\left|\tilde{x}^{\prime}\right|+|y|+\left|y^{\prime}\right|\right)\left(\left|\tilde{x}-\tilde{x}^{\prime}\right|+\left|y-y^{\prime}\right|\right)+C\left(\left|h-h^{\prime}\right|+\left|z-z^{\prime}\right|\right)$,

(ii) $|L(s, \tilde{x}, y, h, z, u, v)| \leq C\left(1+|\tilde{x}|^{2}+|y|^{2}+|h|+|z|\right)$,

(iii) $|F(s, \tilde{x}, y, h, z, u, v)| \leq C\left(1+|\tilde{x}|^{2}+|y|^{2}+|h|+|z|\right)$. 
It is easy to check that $F(s, \tilde{x}, y, h, z, u, v)$ is Lipschitz in $(h, z)$, uniformly with respect to $(s, \tilde{x}, y, u, v)$, and $|F(s, \tilde{x}, 0,0,0, u, v)| \leq C\left(1+|\tilde{x}|^{2}\right),(s, \tilde{x}, u, v) \in[0, T] \times \mathbb{R} \times U \times V$ for some constant $C \geq 0$.

Proof of Lemma 4.3 Let $\left(Y^{u, v}, H^{u, v}, Z^{u, v}\right)$ denote the solution of the BSDE in (4.2). From the definition of the backward semigroup we have,

$$
Y_{s \wedge \tau^{\delta}}^{u, v}=G_{s \wedge \tau^{\delta}, \tau^{\delta}}^{t, x, i u, v}\left[\phi\left(\tau^{\delta}, X_{\tau^{\delta}}^{u, v}\right) \mathbf{1}_{\left\{N_{\tau^{\delta}}^{t, i}=i\right\}}+\sum_{l=1}^{k-1} W_{(l+i) \bmod (k)}\left(\tau^{\delta}, X_{\tau^{\delta}}^{u, v}\right) \mathbf{1}_{\left\{N_{\tau^{\delta}}^{t, i}=(l+i) \bmod (k)\right\}}\right] .
$$

On the other hand,

$$
\begin{aligned}
\tilde{Y}_{\tau^{\delta}}^{1, i, u, v} & =\phi\left(\tau^{\delta}, X_{\tau^{\delta}}^{u, v}\right)+\sum_{l=1}^{k-1}\left(W_{(l+i) \bmod (k)}\left(\tau^{\delta}, X_{\tau^{\delta}}^{u, v}\right)-\phi\left(\tau^{\delta}, X_{\tau^{\delta}}^{u, v}\right)\right) \Delta N_{\tau^{\delta}}(l) \\
& =\phi\left(\tau^{\delta}, X_{\tau^{\delta}}^{u, v}\right) \mathbf{1}_{\left\{N_{\tau^{\delta}}^{t, i}=i\right\}}+\sum_{l=1}^{k-1} W_{(l+i) \bmod (k)}\left(\tau^{\delta}, X_{\tau^{\delta}}^{u, v}\right) \mathbf{1}_{\left\{N_{\tau^{\delta}}^{t, i}=(l+i) \bmod (k)\right\}} \\
& =Y_{\tau^{\delta}}^{u, v},(\operatorname{see}(4.2)) .
\end{aligned}
$$

Moreover, applying Itô's formula to $\tilde{Y}_{s}^{1, i, u, v}-Y_{s}^{u, v}$ and using (4.2), we get

$$
\begin{aligned}
d\left(\tilde{Y}_{s}^{1, i, u, v}-Y_{s}^{u, v}\right)= & \frac{\partial \phi}{\partial s}\left(s, X_{s}^{u, v}\right)+\frac{1}{2} \operatorname{tr}\left(\sigma_{i} \sigma_{i}^{*}\left(s, X_{s}^{u, v}, Y_{s}^{u, v}, u_{s}, v_{s}\right) D^{2} \phi\left(s, X_{s}^{u, v}\right)\right) \\
& \left.+b_{i}\left(s, X_{s}^{u, v}, Y_{s}^{u, v}, Z_{s}^{u, v}, u_{s}, v_{s}\right) D \phi\left(s, X_{s}^{u, v}\right)+\tilde{f}_{i}\left(s, X_{s}^{u, v}, Y_{s}^{u, v}, H_{s}^{u, v}, Z_{s}^{u, v}, u_{s}, v_{s}\right)\right] \mathrm{d} s \\
& -\lambda \sum_{l=1}^{k-1}\left[H_{s}^{u, v}(l)-W_{(l+i) \bmod (k)}\left(s, X_{s}^{u, v}\right)+\phi\left(s, X_{s}^{u, v}\right)\right] \mathrm{d} s \\
& -\left[Z_{s}^{u, v}-D \phi\left(s, X_{s}^{u, v}\right) \sigma_{i}\left(s, X_{s}^{u, v}, Y_{s}^{u, v}, u_{s}, v_{s}\right)\right] \mathrm{d} B_{s} \\
& -\sum_{l=1}^{k-1}\left[H_{s}^{u, v}(l)-W_{(l+i) \bmod (k)}\left(s, X_{s}^{u, v}\right)+\phi\left(s, X_{s}^{u, v}\right)\right] \mathrm{d} \tilde{N}_{s}(l) .
\end{aligned}
$$

By setting

$$
\begin{aligned}
& Y_{s}^{1, i, u, v}=Y_{s}^{u, v}-\tilde{Y}_{s}^{1, i, u, v} \\
& Z_{s}^{1, i, u, v}=Z_{s}^{u, v}-D \phi\left(s, X_{s}^{u, v}\right) \sigma_{i}\left(s, X_{s}^{u, v}, Y_{s}^{u, v}, u_{s}, v_{s}\right), \\
& H_{s}^{1, i, u, v}(l)=H_{s}^{u, v}(l)-W_{(l+i) \bmod (k)}\left(s, X_{s}^{u, v}\right)+\phi\left(s, X_{s}^{u, v}\right), 1 \leq l \leq k-1, s \in\left[t, \tau^{\delta}\right],
\end{aligned}
$$

we get BSDE (4.5). The uniqueness of the solution $\left(X^{u, v}, Y^{u, v}, H^{u, v}, Z^{u, v}\right)$ of (4.2) gives the stated result.

Lemma 4.5. For some $C \in \mathbb{R}_{+}$independent of $\delta>0$, it holds

$$
\mathbb{E}\left[\int_{t}^{\tau^{\delta}}\left(\left|Y_{s}^{1, i, u, v}\right|+\lambda \sum_{l=1}^{k-1}\left|H_{s}^{1, i, u, v}(l)\right|+\left|Z_{s}^{1, i, u, v}\right|\right) \mathrm{d} s \mid \mathcal{F}_{t}\right] \leq C \delta^{\frac{5}{4}}, \quad P \text {-a.s. },
$$

for all $\delta \in\left(0, \delta_{0}\right)$, and all $u \in \mathcal{U}_{t, \tau^{\delta}}, v \in \mathcal{V}_{t, \tau^{\delta}}$, where $\delta_{0}>0$ is small enough . 
Proof. From (4.7), (4.6) and (4.4), we know

$$
\begin{aligned}
Y_{s \wedge \tau^{\delta}}^{1, i, u, v}= & Y_{s \wedge \tau^{\delta}}^{u, v}-\tilde{Y}_{s \wedge \tau^{\delta}}^{1, i, u, v} \\
= & -\mathbb{E}\left[Y_{\tau^{\delta}}^{u, v}-Y_{s \wedge \tau^{\delta}}^{u, v} \mid \mathcal{F}_{s \wedge \tau^{\delta}}\right]+\mathbb{E}\left[\tilde{Y}_{\tau^{\delta}}^{1, i, u, v}-\tilde{Y}_{s \wedge \tau^{\delta}}^{1, i, u, v} \mid \mathcal{F}_{s \wedge \tau^{\delta}}\right] \\
= & \mathbb{E}\left[\int_{s \wedge \tau^{\delta}}^{\tau^{\delta}} \tilde{f}_{i}\left(r, X_{r}^{u, v}, Y_{r}^{u, v}, H_{r}^{u, v}, Z_{r}^{u, v}, u_{r}, v_{r}\right) d r-\int_{s \wedge \tau^{\delta}}^{\tau^{\delta}} \lambda \sum_{l=1}^{k} H_{r}^{u, v}(l) d r \mid \mathcal{F}_{s \wedge \tau^{\delta}}\right] \\
& +\mathbb{E}\left[\phi\left(\tau^{\delta}, X_{\tau^{\delta}}^{u, v}\right)-\phi\left(s \wedge \tau^{\delta}, X_{s \wedge \tau^{\delta}}^{u, v}\right) \mid \mathcal{F}_{s \wedge \tau^{\delta}}\right] \\
& +\mathbb{E}\left[\sum_{l=1}^{k-1} \int_{s \wedge \tau^{\delta}}^{\tau^{\delta}}\left(W_{(l+i) \bmod (k)}\left(r, X_{r}^{u, v}\right)-\phi\left(r, X_{r}^{u, v}\right)\right) d N_{r}(l) \mid \mathcal{F}_{s \wedge \tau^{\delta}}\right], \quad s \in[t, t+\delta] .
\end{aligned}
$$

Therefore, from (4.3)

$$
\begin{aligned}
& \left|Y_{s \wedge \tau^{\delta}}^{1, i, u, v}\right| \leq C \mathbb{E}\left[\int_{s \wedge \tau^{\delta}}^{\tau^{\delta}}\left(1+\left|X_{r}^{u, v}\right|+\left|Y_{r}^{u, v}\right|+\sum_{l=1}^{k-1}\left|H_{r}^{u, v}(l)\right|+\left|Z_{r}^{u, v}\right|\right) d r \mid \mathcal{F}_{s \wedge \tau^{\delta}}\right] \\
& +C \mathbb{E}\left[\left|\phi\left(\tau^{\delta}, X_{\tau^{\delta}}^{u, v}\right)-\phi\left(s \wedge \tau^{\delta}, X_{s \wedge \tau^{\delta}}^{u, v}\right)\right| \mid \mathcal{F}_{s \wedge \tau^{\delta}}\right] \\
& +\left|\mathbb{E}\left[\sum_{l=1}^{k-1} \int_{s \wedge \tau^{\delta}}^{\tau^{\delta}}\left(W_{(l+i) \bmod (k)}\left(r, X_{r}^{u, v}\right)-\phi\left(r, X_{r}^{u, v}\right)\right) d N_{r}(l) \mid \mathcal{F}_{s \wedge \tau^{\delta}}\right]\right| \\
& \leq C \mathbb{E}\left[\int_{s \wedge \tau^{\delta}}^{\tau^{\delta}}\left(1+\left|X_{r}^{u, v}\right|+\left|Y_{r}^{u, v}\right|+\sum_{l=1}^{k-1}\left|H_{r}^{u, v}(l)\right|+\left|Z_{r}^{u, v}\right|\right) d r \mid \mathcal{F}_{s \wedge \tau^{\delta}}\right] \\
& +C \delta+C \mathbb{E}\left[\left|X_{\tau^{\delta}}^{u, v}-X_{s \wedge \tau^{\delta}}^{u, v}\right| \mid \mathcal{F}_{s \wedge \tau^{\delta}}\right] \\
& +\left|\lambda \mathbb{E}\left[\sum_{l=1}^{k-1} \int_{s \wedge \tau^{\delta}}^{\tau^{\delta}}\left(W_{(l+i) \bmod (k)}\left(r, X_{r}^{u, v}\right)-\phi\left(r, X_{r}^{u, v}\right)\right) d r \mid \mathcal{F}_{s \wedge \tau^{\delta}}\right]\right| \\
& \leq C \delta^{\frac{1}{2}}\left(\mathbb{E}\left[\int_{s \wedge \tau^{\delta}}^{t+\delta}\left(1+\left|X_{r}^{u, v}\right|^{2}+\left|Y_{r}^{u, v}\right|^{2}+\sum_{l=1}^{k-1}\left|H_{r}^{u, v}(l)\right|^{2}+\left|Z_{r}^{u, v}\right|^{2}\right) d r \mid \mathcal{F}_{s \wedge \tau^{\delta}}\right]\right)^{\frac{1}{2}} \\
& +C \delta+C \delta^{\frac{1}{2}}\left(1+\left|X_{s \wedge \tau^{\delta}}^{u, v}\right|\right) \\
& \leq C \delta^{\frac{1}{2}}\left(1+\left|X_{s \wedge \tau^{\delta}}^{u, v}\right|\right), \quad \text { P-a.s., } s \in[t, t+\delta] \text {. }
\end{aligned}
$$

From (4.7), we get

$$
\begin{aligned}
& \left|Z_{s}^{1, i, u, v}\right| \leq C\left(1+\left|X_{s}^{u, v}\right|+\left|Y_{s}^{u, v}\right|+\left|Z_{s}^{u, v}\right|\right), \\
& \sum_{l=1}^{k-1}\left|H_{s}^{1, i, u, v}(l)\right| \leq C\left(1+\left|X_{s}^{u, v}\right|+\sum_{l=1}^{k-1}\left|H_{s}^{u, v}(l)\right|\right), \text { P-a.s., } s \in\left[t, \tau^{\delta}\right] .
\end{aligned}
$$


Applying Itô's formula to $\left|Y_{s}^{1, i, u, v}\right|^{2}$, from (4.5) we obtain

$$
\begin{aligned}
& \left|Y_{t}^{1, i, u, v}\right|^{2}+\mathbb{E}\left[\int_{t}^{\tau^{\delta}}\left|Z_{r}^{1, i, u, v}\right|^{2} d r+\lambda \int_{t}^{\tau^{\delta}} \sum_{l=1}^{k-1}\left|H_{r}^{1, i, u, v}(l)\right|^{2} d r \mid \mathcal{F}_{t}\right] \\
= & 2 \mathbb{E}\left[\int_{t}^{\tau^{\delta}} Y_{r}^{1, i, u, v} F\left(r, X_{r}^{u, v}, Y_{r}^{1, i, u, v}, H_{r}^{1, i, u, v}, Z_{r}^{1, i, u, v}, u_{r}, v_{r}\right) d r \mid \mathcal{F}_{t}\right] \\
& -\mathbb{E}\left[\int_{t}^{\tau^{\delta}} 2 \lambda Y_{r}^{1, i, u, v} \sum_{l=1}^{k-1} H_{r}^{1, i, u, v}(l) d r \mid \mathcal{F}_{t}\right] \\
\leq & 2 \mathbb{E}\left[\int_{t}^{\tau^{\delta}} Y_{r}^{1, i, u, v} F\left(r, X_{r}^{u, v}, Y_{r}^{1, i, u, v}, H_{r}^{1, i, u, v}, Z_{r}^{1, i, u, v}, u_{r}, v_{r}\right) d r \mid \mathcal{F}_{t}\right] \\
& +C \mathbb{E}\left[\int_{t}^{\tau^{\delta}}\left|Y_{r}^{1, i, u, v}\right|^{2} d r \mid \mathcal{F}_{t}\right]+\frac{\lambda}{2} E\left[\int_{t}^{\tau^{\delta}} \sum_{l=1}^{k-1}\left|H_{r}^{1, i, u, v}(l)\right|^{2} d r \mid \mathcal{F}_{t}\right] .
\end{aligned}
$$

Thus, combing this estimate with Remark 4.1, (4.9), (4.10) as well as (4.3) yields

$$
\begin{aligned}
& \left|Y_{t}^{1, i, u, v}\right|^{2}+\mathbb{E}\left[\int_{t}^{\tau^{\delta}}\left|Z_{r}^{1, i, u, v}\right|^{2} d r+\lambda \int_{t}^{\tau^{\delta}} \sum_{l=1}^{k-1}\left|H_{r}^{1, i, u, v}(l)\right|^{2} d r \mid \mathcal{F}_{t}\right] \\
\leq & 4 \mathbb{E}\left[\int_{t}^{\tau^{\delta}} Y_{r}^{1, i, u, v} F\left(r, X_{r}^{u, v}, Y_{r}^{1, i, u, v}, H_{r}^{1, i, u, v}, Z_{r}^{1, i, u, v}, u_{r}, v_{r}\right) d r \mid \mathcal{F}_{t}\right]+C \mathbb{E}\left[\int_{t}^{\tau^{\delta}}\left|Y_{r}^{1, i, u, v}\right|^{2} d r \mid \mathcal{F}_{t}\right] \\
\leq & C \mathbb{E}\left[\int_{t}^{\tau^{\delta}}\left|Y_{r}^{1, i, u, v}\right|\left(1+\left|X_{r}^{u, v}\right|^{2}+\left|Y_{r}^{1, i, u, v}\right|^{2}+\left|Z_{r}^{1, i, u, v}\right|+\sum_{l=1}^{k-1}\left|H_{r}^{1, i, u, v}(l)\right|\right) d r \mid \mathcal{F}_{t}\right] \\
& +C \mathbb{E}\left[\int_{t}^{\tau^{\delta}}\left|Y_{r}^{1, i, u, v}\right|^{2} d r \mid \mathcal{F}_{t}\right] \\
\leq & C \delta^{\frac{1}{2}} \mathbb{E}\left[\int_{t}^{\tau^{\delta}}\left(1+\left|X_{r}^{u, v}\right|^{2}+\left|X_{r}^{u, v}\right|^{3}\right) d r \mid \mathcal{F}_{t}\right]+C \mathbb{E}\left[\int_{t}^{\tau^{\delta}}\left|Y_{r}^{1, i, u, v}\right|^{3} d r \mid \mathcal{F}_{t}\right] \\
& +C \delta^{\frac{1}{2}} \mathbb{E}\left[\int_{t}^{\tau^{\delta}}\left(1+\left|X_{r}^{u, v}\right|\right)\left|Y_{r}^{u, v}\right| d r \mid \mathcal{F}_{t}\right]+C \delta^{\frac{1}{2}} \mathbb{E}\left[\int_{t}^{\tau^{\delta}}\left(1+\left|X_{r}^{u, v}\right|\right)\left|Z_{r}^{u, v}\right| d r \mid \mathcal{F}_{t}\right] \\
& +C \delta^{\frac{1}{2}} \mathbb{E}\left[\int_{t}^{\tau^{\delta}}\left(1+\left|X_{r}^{u, v}\right|\right) \sum_{l=1}^{k-1}\left|H_{r}^{u, v}(l)\right| d r \mid \mathcal{F}_{t}\right]+C \delta \mathbb{E}\left[\int_{t}^{\tau^{\delta}}\left(1+\left|X_{r}^{u, v}\right|^{2}\right) d r \mid \mathcal{F}_{t}\right] \\
\leq & C \delta^{\frac{3}{2}} .
\end{aligned}
$$

Therefore, from (4.9), (4.12) and (4.3)-(i) we have

$$
\begin{aligned}
& \mathbb{E}\left[\int_{t}^{\tau^{\delta}}\left(\left|Y_{r}^{1, i, u, v}\right|+\left|Z_{r}^{1, i, u, v}\right|+\lambda \sum_{l=1}^{k-1}\left|H_{r}^{1, i, u, v}(l)\right|\right) d r \mid \mathcal{F}_{t}\right] \\
\leq & C \delta^{\frac{1}{2}} \mathbb{E}\left[\int_{t}^{\tau^{\delta}}\left(1+\left|X_{r}^{u, v}\right|\right) d r \mid \mathcal{F}_{t}\right]+C \delta^{\frac{1}{2}}\left(\mathbb{E}\left[\int_{t}^{\tau^{\delta}}\left|Z_{r}^{1, i, u, v}\right|^{2} d r \mid \mathcal{F}_{t}\right]\right)^{\frac{1}{2}} \\
& +C \delta^{\frac{1}{2}}\left(\mathbb{E}\left[\int_{t}^{\tau^{\delta}} \sum_{l=1}^{k-1}\left|H_{r}^{1, i, u, v}(l)\right|^{2} d r \mid \mathcal{F}_{t}\right]\right)^{\frac{1}{2}} \\
\leq & C \delta^{\frac{5}{4}}, \text { P-a.s. }
\end{aligned}
$$


Let us focus now on the following BSDE with jumps:

$$
\left\{\begin{aligned}
\mathrm{d} Y_{s}^{2, i, u, v}= & -F\left(s, x, 0, H_{s}^{2, i, u, v}, Z_{s}^{2, i, u, v}, u_{s}, v_{s}\right) \mathrm{d} s+\lambda \sum_{l=1}^{k-1} H_{s}^{2, i, u, v}(l) \mathrm{d} s \\
& +Z_{s}^{2, i, u, v} \mathrm{~d} B_{s}+\sum_{l=1}^{k-1} H_{s}^{2, i, u, v}(l) d \tilde{N}_{s}(l), \quad s \in\left[t, \tau^{\delta}\right], \\
Y_{\tau^{\delta}}^{2, i, u, v}= & 0 .
\end{aligned}\right.
$$

Lemma 4.6. There is some $C \in \mathbb{R}_{+}$such that, for all $\delta \in\left(0, \delta_{0}\right)$ and for all $u \in \mathcal{U}_{t, \tau^{\delta}}, v \in \mathcal{V}_{t, \tau^{\delta}}$, where $\delta_{0}$ is small enough, it holds

(i) $\left|Y_{t}^{1, i, u, v}-Y_{t}^{2, i, u, v}\right| \leq C \delta^{\frac{4}{3}}, \quad P$-a.s.

(ii) $\mathbb{E}\left[\int_{t}^{\tau^{\delta}}\left(\lambda \sum_{l=1}^{k-1}\left|H_{s}^{1, i, u, v}(l)-H_{s}^{2, i, u, v}(l)\right|^{2}+\left|Z_{s}^{1, i, u, v}-Z_{s}^{2, i, u, v}\right|^{2}\right) \mathrm{d} s \mid \mathcal{F}_{t}\right] \leq C \delta^{\frac{5}{3}}, \quad P$-a.s.

Proof. As $F(s, \tilde{x}, y, h, z, u, v)$ is Lipschitz in $(h, z)$, uniformly with respect to $(s, \tilde{x}, y, u, v)$, a classical BSDE estimate yields

$$
\begin{aligned}
& \left|Y_{t}^{1, i, u, v}-Y_{t}^{2, i, u, v}\right|^{2}+\mathbb{E}\left[\int_{t}^{\tau^{\delta}}\left(\lambda \sum_{l=1}^{k-1}\left|H_{s}^{1, i, u, v}(l)-H_{s}^{2, i, u, v}(l)\right|^{2}+\left|Z_{s}^{1, i, u, v}-Z_{s}^{2, i, u, v}\right|^{2}\right) \mathrm{d} s \mid \mathcal{F}_{t}\right] \\
\leq & \mathbb{E}\left[\int_{t}^{\tau^{\delta}}\left|F\left(s, X_{s}^{u, v}, Y_{s}^{1, i, u, v}, H_{s}^{1, i, u, v}, Z_{s}^{1, i, u, v}, u_{s}, v_{s}\right)-F\left(s, x, 0, H_{s}^{1, i, u, v}, Z_{s}^{1, i, u, v}, u_{s}, v_{s}\right)\right|^{2} d s \mid \mathcal{F}_{t}\right] \\
\leq & C \mathbb{E}\left[\int_{t}^{\tau^{\delta}}\left(1+|x|+\left|X_{s}^{u, v}\right|+\left|Y_{s}^{1, i, u, v}\right|\right)^{2}\left(\left|X_{s}^{u, v}-x\right|+\left|Y_{s}^{1, i, u, v}\right|\right)^{2} \mathrm{~d} s \mid \mathcal{F}_{t}\right] .
\end{aligned}
$$

Consequently, from (4.3) (i)-(ii) and (4.9),

$$
\begin{aligned}
& \left|Y_{t}^{1, i, u, v}-Y_{t}^{2, i, u, v}\right|^{2}+\mathbb{E}\left[\int_{t}^{\tau^{\delta}}\left(\lambda \sum_{l=1}^{k-1}\left|H_{s}^{1, i, u, v}(l)-H_{s}^{2, i, u, v}(l)\right|^{2}+\left|Z_{s}^{1, i, u, v}-Z_{s}^{2, i, u, v}\right|^{2}\right) \mathrm{d} s \mid \mathcal{F}_{t}\right] \\
\leq & C \delta \mathbb{E}\left[\sup _{s \in\left[t, \tau^{\delta}\right]}\left(1+\left|X_{s}^{u, v}\right|\right)^{2}\left(\left|X_{s}^{u, v}-x\right|^{2}+\delta\left(1+\left|X_{s}^{u, v}\right|\right)^{2}\right) \mid \mathcal{F}_{t}\right] \\
\leq & C \delta\left(\mathbb{E}\left[\sup _{s \in\left[t, \tau^{\delta}\right]}\left(1+\left|X_{s}^{u, v}\right|\right)^{6} \mid \mathcal{F}_{t}\right]\right)^{\frac{1}{3}}\left(\mathbb{E}\left[\sup _{s \in\left[t, \tau^{\delta}\right]}\left(\left|X_{s}^{u, v}-x\right|^{3}+\delta^{\frac{3}{2}}\left(1+\left|X_{s}^{u, v}\right|\right)^{3}\right) \mid \mathcal{F}_{t}\right]\right)^{\frac{2}{3}} \\
\leq & C \delta\left(\delta^{\frac{2}{3}}+\delta\right) \leq C \delta^{\frac{5}{3}}
\end{aligned}
$$


for all $(u, v)$ and all $\delta \in\left(0, \delta_{0}\right)$, for some $\delta_{0}>0$ sufficiently small. On the other hand,

$$
\begin{aligned}
& \left|Y_{t}^{1, i, u, v}-Y_{t}^{2, i, u, v}\right| \\
= & \mid \mathbb{E}\left[\int_{t}^{\tau^{\delta}}\left(F\left(s, X_{s}^{u, v}, Y_{s}^{1, i, u, v}, H_{s}^{1, i, u, v}, Z_{s}^{1, i, u, v}, u_{s}, v_{s}\right)-F\left(s, x, 0, H_{s}^{2, i, u, v}, Z_{s}^{2, i, u, v}, u_{s}, v_{s}\right)\right) \mathrm{d} s \mid \mathcal{F}_{t}\right] \\
& -\lambda \mathbb{E}\left[\int_{t}^{\tau^{\delta}} \sum_{l=1}^{k-1}\left(H_{s}^{1, i, u, v}(l)-H_{s}^{2, i, u, v}(l)\right) \mathrm{d} s \mid \mathcal{F}_{t}\right] \mid \\
\leq & C \mathbb{E}\left[\int _ { t } ^ { \tau ^ { \delta } } \left(\left(1+|x|+\left|X_{s}^{u, v}\right|+\left|Y_{s}^{1, i, u, v}\right|\right)\left(\left|X_{s}^{u, v}-x\right|+\left|Y_{s}^{1, i, u, v}\right|\right)\right.\right. \\
& \left.\left.+\lambda \sum_{l=1}^{k-1}\left|H_{s}^{1, i, u, v}(l)-H_{s}^{2, i, u, v}(l)\right|+\left|Z_{s}^{1, i, u, v}-Z_{s}^{2, i, u, v}\right|\right) \mathrm{d} s \mid \mathcal{F}_{t}\right] \\
\leq & C \mathbb{E}\left[\int_{t}^{\tau^{\delta}}\left(\left|X_{s}^{u, v}-x\right|+\left|Y_{s}^{1, i, u, v}\right|+\left|X_{s}^{u, v}-x\right|^{2}+\left|Y_{s}^{1, i, u, v}\right|^{2}\right) \mathrm{d} s \mid \mathcal{F}_{t}\right] \\
& +C \delta^{\frac{1}{2}}\left(\mathbb{E}\left[\int_{t}^{\tau^{\delta}}\left(\lambda \sum_{l=1}^{k-1}\left|H_{s}^{1, i, u, v}(l)-H_{s}^{2, i, u, v}(l)\right|^{2}+\left|Z_{s}^{1, i, u, v}-Z_{s}^{2, i, u, v}\right|^{2}\right) \mathrm{d} s \mid \mathcal{F}_{t}\right]\right)^{\frac{1}{2}},
\end{aligned}
$$

and from (4.9) as well as (4.3) and (4.16) we obtain

$$
\begin{aligned}
& \left|Y_{t}^{1, i, u, v}-Y_{t}^{2, i, u, v}\right| \\
\leq & C \delta\left(\mathbb{E}\left[\sup _{s \in\left[t, \tau^{\delta}\right]}\left(\left|X_{s}^{u, v}-x\right|^{2}+\left|X_{s}^{u, v}-x\right|^{4}\right) \mid \mathcal{F}_{t}\right]\right)^{\frac{1}{2}}+C \delta^{\frac{3}{2}} \mathbb{E}\left[\sup _{s \in\left[t, \tau^{\delta}\right]}\left(1+\left|X_{s}^{u, v}\right|^{2}\right) \mid \mathcal{F}_{t}\right] \\
& +C \delta^{\frac{1}{2}}\left(\mathbb{E}\left[\int_{t}^{\tau^{\delta}}\left(\lambda \sum_{l=1}^{k-1}\left|H_{s}^{1, i, u, v}(l)-H_{s}^{2, i, u, v}(l)\right|^{2}+\left|Z_{s}^{1, i, u, v}-Z_{s}^{2, i, u, v}\right|^{2}\right) \mathrm{d} s \mid \mathcal{F}_{t}\right]\right)^{\frac{1}{2}} \\
\leq & C \delta^{\frac{3}{2}}+C \delta^{\frac{4}{3}} \leq C \delta^{\frac{4}{3}}, \text { P-a.s. }
\end{aligned}
$$

Lemma 4.7. Let $Y^{3, i}$ be the solution of the following equation:

$$
\left\{\begin{array}{l}
\mathrm{d} Y_{s}^{3, i}=-F_{0}\left(s, x, 0, H_{s}^{3, i}, 0\right) \mathrm{d} s+\sum_{l=1}^{k-1} H_{s}^{3, i}(l)\left[\mathrm{d} \tilde{N}_{s}(l)+\lambda \mathrm{d} s\right], \quad s \in\left[t, \tau^{\delta}\right] \\
Y_{\tau^{\delta}}^{3, i}=0
\end{array}\right.
$$

where the function $F_{0}$ is defined by

$$
F_{0}(s, x, y, h, z)=\sup _{u \in U} \inf _{v \in V} F(s, x, y, h, z, u, v)
$$

Then, there is some $\delta_{0}>0$ small enough such that, for all $\delta \in\left(0, \delta_{0}\right)$, P-a.s.,

$$
\underset{u \in \mathcal{U}_{t, \tau \delta}}{\operatorname{essup}} \underset{v \in \mathcal{V}_{t, \tau} \delta}{\operatorname{essinf}} Y_{t}^{2, i, u, v}=Y_{t}^{3, i}
$$


Proof. Let us define $\hat{F}(s, x, y, h, z, u)=\inf _{v \in V} F(s, x, y, h, z, u, v),(s, x, y, h, z, u) \in[0, T] \times \mathbb{R} \times \mathbb{R} \times \mathbb{R}^{k-1} \times \mathbb{R}^{d} \times U$, and consider, for $u \in \mathcal{U}_{t, \tau^{\delta}}$, the following BSDE:

$$
\left\{\begin{array}{l}
\mathrm{d} Y_{s}^{3, i, u}=-\hat{F}\left(s, x, 0, H_{s}^{3, i, u}, Z_{s}^{3, i, u}, u_{s}\right) \mathrm{d} s+\sum_{l=1}^{k-1} H_{s}^{3, i, u}(l)\left(\mathrm{d} \tilde{N}_{s}(l)+\lambda \mathrm{d} s\right)+Z_{s}^{3, i, u} \mathrm{~d} B_{s}, s \in\left[t, \tau^{\delta}\right], \\
Y_{\tau^{\delta}}^{3, i, u}=0
\end{array}\right.
$$

for all $\delta \in\left(0, \delta_{0}\right]$. It is clear that there exists a unique solution $\left(Y_{s}^{3, i, u}, H_{s}^{3, i, u}, Z_{s}^{3, i, u}\right)$ to (4.20). Moreover,

$$
Y_{t}^{3, i, u}=\underset{v \in \mathcal{V}_{t, \tau^{\delta}}}{\operatorname{essinf}} Y_{t}^{2, i, u, v}, \text { P-a.s., for all } u \in \mathcal{U}_{t, \tau^{\delta}}
$$

Indeed, from the definition of $\hat{F}$ and the comparison theorem for BSDEs with jumps (see Thm. 2.5 in [11]), we have

$$
Y_{t}^{3, i, u} \leq \operatorname{essinf}_{v \in \mathcal{V}_{t, \tau \delta}} Y_{t}^{2, i, u, v}, \text { P-a.s., for all } u \in \mathcal{U}_{t, \tau^{\delta}}
$$

On the other hand, there exists a Borel measurable function $v^{2}:[0, T] \times \mathbb{R} \times \mathbb{R}^{k-1} \times \mathbb{R}^{d} \times U \rightarrow V$ such that

$$
\hat{F}(s, x, 0, h, z, u)=F\left(s, x, 0, h, z, u, v^{2}(s, x, h, z, u)\right) \text {, for any }(s, x, h, z, u) \text {. }
$$

By setting $\widetilde{v}_{s}^{2}=v^{2}\left(s, x, H_{s}^{3, i, u}, Z_{s}^{3, i, u}, u_{s}\right), s \in\left[t, \tau^{\delta}\right]$, we see $\widetilde{v}^{2} \in \mathcal{V}_{t, \tau^{\delta}}$, and

$$
\hat{F}\left(s, x, 0, H_{s}^{3, i, u}, Z_{s}^{3, i, u}, u_{s}\right)=F\left(s, x, 0, H_{s}^{3, i, u}, Z_{s}^{3, i, u}, u_{s}, \widetilde{v}_{s}^{2}\right), s \in\left[t, \tau^{\delta}\right] .
$$

From the uniqueness of the solution of the BSDE with jumps, we conclude that

$$
\left(Y^{3, i, u}, H^{3, i, u}, Z^{3, i, u}\right)=\left(Y^{2, i, u, \widetilde{v}^{2}}, H^{2, i, u, \widetilde{v}^{2}}, Z^{2, i, u, \widetilde{v}^{2}}\right),
$$

and in particular, $Y_{t}^{3, i, u}=Y_{t}^{2, i, u, \widetilde{v}^{2}}$, P-a.s., for all $u \in \mathcal{U}_{t, \tau^{\delta}}$. Consequently, $Y_{t}^{3, i, u}=\underset{v \in \mathcal{V}_{t, \tau^{\delta}}}{\operatorname{essinf}} Y_{t}^{2, i, u, v}$, P-a.s., for all $u \in \mathcal{U}_{t, \tau^{\delta}}$.

Finally, as $F_{0}(s, x, y, h, z)=\sup _{u \in U} \hat{F}(s, x, y, h, z, u)$, by a similar argument we get the desired result:

$$
Y_{t}^{3, i}=\operatorname{esssup}_{u \in \mathcal{U}_{t, \tau^{\delta}}} Y_{t}^{3, i, u}=\operatorname{esssup}_{u \in \mathcal{U}_{t, \tau^{\delta}}} \operatorname{essinf}_{v \in \mathcal{V}_{t, \tau^{\delta}}} Y_{t}^{2, i, u, v}, \text { P-a.s. }
$$

Remark 4.8. Notice that the solution $\left(Y^{3, i}, H^{3, i}\right)$ of (4.17) is independent of the Brownian motion $B$ but still depends on the Poisson random measure $N$.

Now we extend equation (4.17) to the interval $[t, t+\delta]$ as follows:

$$
\left\{\begin{array}{l}
d Y_{s}^{0, \delta}=-\mathbf{1}_{\left[t, \tau^{\delta}\right]}(s) F_{0}\left(s, x, 0, H_{s}^{0, \delta}, 0\right) \mathrm{d} s+\sum_{l=1}^{k-1} H_{s}^{0, \delta}(l)\left[d \tilde{N}_{s}(l)+\lambda \mathrm{d} s\right], s \in[t, t+\delta], \\
Y_{t+\delta}^{0, \delta}=0 .
\end{array}\right.
$$


Remark 4.9. It is easy to check that

$$
Y_{s}^{0, \delta}=\left\{\begin{array}{ll}
Y_{s}^{3, i}, & s \in\left[t, \tau^{\delta}\right], \\
0, & s \in\left[\tau^{\delta}, t+\delta\right],
\end{array} \quad H_{s}^{0, \delta}= \begin{cases}H_{s}^{3, i}, & s \in\left[t, \tau^{\delta}\right], \\
0, & s \in\left[\tau^{\delta}, t+\delta\right],\end{cases}\right.
$$

So we have $Y_{t}^{0, \delta}=Y_{t}^{3, i}=\underset{u \in \mathcal{U}_{t, \tau^{\delta}}}{\operatorname{esssup}} \operatorname{essinf}_{v \in \mathcal{V}_{t, \tau^{\delta}}} Y_{t}^{2, i, u, v}, P$-a.s.

Furthermore, we consider

$$
\left\{\begin{array}{l}
d \bar{Y}_{s}^{0, \delta}=-F_{0}(s, x, 0,0,0) \mathrm{d} s, s \in[t, t+\delta] \\
\bar{Y}_{t+\delta}^{0, \delta}=0
\end{array}\right.
$$

and we can estimate the difference between $Y_{t}^{0, \delta}$ and $\bar{Y}_{t}^{0, \delta}$ as follows:

Lemma 4.10. There exists a constant $C \in \mathbb{R}_{+}$such that for all $\delta \in\left(0, \delta_{0}\right)$, for some $\delta_{0}>0$,

$$
\left|Y_{t}^{0, \delta}-\bar{Y}_{t}^{0, \delta}\right| \leq C \delta^{\frac{3}{2}}, P-a . s .
$$

Proof. We notice that

$$
\left\{\begin{array}{l}
d \bar{Y}_{s}^{0, \delta}=-F_{0}\left(s, x, 0, \bar{H}_{s}^{0, \delta}, 0\right) \mathrm{d} s+\sum_{l=1}^{k-1} \bar{H}_{s}^{0, \delta}(l)\left[d \tilde{N}_{s}(l)+\lambda \mathrm{d} s\right], s \in[t, t+\delta] \\
\bar{Y}_{t+\delta}^{0, \delta}=0
\end{array}\right.
$$

where $\bar{H}_{s}^{0, \delta}=0, s \in[t, t+\delta]$.

On the other hand, from $(4.22), \bar{Y}_{\tau^{\delta}}^{0, \delta}=\int_{\tau^{\delta}}^{t+\delta} F_{0}(s, x, 0,0,0) \mathrm{d} s$, and as $\left|F_{0}(s, x, 0,0,0)\right| \leq C\left(1+|x|^{2}\right)=C$ (indeed, $x$ is fixed), we have from the definition of $\tau^{\delta}$,

$$
\left|\bar{Y}_{\tau^{\delta}}^{0, \delta}\right| \leq C\left(t+\delta-\tau^{\delta}\right) \leq \sum_{l=1}^{k-1} C \delta \mathbf{1}_{\{N((t, t+\delta] \times\{l\}) \geq 1\}},
$$

that is,

$$
\mathbb{E}\left[\left|\bar{Y}_{\tau^{\delta}}^{0, \delta}\right|^{2} \mid \mathcal{F}_{t}\right] \leq C k^{2} \delta^{2}(1-P(N((t, t+\delta] \times\{l\})=0))=C k^{2} \delta^{2}\left(1-e^{-\lambda \delta}\right) \leq C \lambda k^{2} \delta^{3}
$$

As we have

$$
\left\{\begin{array}{l}
d \bar{Y}_{s}^{0, \delta}=-F_{0}\left(s, x, 0, \bar{H}_{s}^{0, \delta}, 0\right) \mathrm{d} s+\sum_{l=1}^{k-1} \bar{H}_{s}^{0, \delta}(l)\left[d \tilde{N}_{s}(l)+\lambda \mathrm{d} s\right], s \in\left[t, \tau^{\delta}\right] \\
\bar{Y}_{\tau^{\delta}}^{0, \delta}=\int_{\tau^{\delta}}^{t+\delta} F_{0}(s, x, 0,0,0) \mathrm{d} s
\end{array}\right.
$$

and

$$
\left\{\begin{array}{l}
d Y_{s}^{0, \delta}=-F_{0}\left(s, x, 0, H_{s}^{0, \delta}, 0\right) \mathrm{d} s+\sum_{l=1}^{k-1} H_{s}^{0, \delta}(l)\left[d \tilde{N}_{s}(l)+\lambda \mathrm{d} s\right], s \in\left[t, \tau^{\delta}\right] \\
Y_{\tau^{\delta}}^{0, \delta}=0
\end{array}\right.
$$


we get from a BSDE standard estimate,

$$
\left|\bar{Y}_{t}^{0, \delta}-Y_{t}^{0, \delta}\right|^{2}+\mathbb{E}\left[\lambda \int_{t}^{\tau^{\delta}} \sum_{l=1}^{k-1}\left|\bar{H}_{s}^{0, \delta}(l)-H_{s}^{0, \delta}(l)\right|^{2} \mathrm{~d} s \mid \mathcal{F}_{t}\right] \leq C \mathbb{E}\left[\left|\bar{Y}_{\tau^{\delta}}^{0, \delta}-Y_{\tau^{\delta}}^{0, \delta}\right|^{2} \mid \mathcal{F}_{t}\right] \leq C \delta^{3}, \delta \in\left(0, \delta_{0}\right), P \text {-a.s. }
$$

Consequently,

$$
\left|\bar{Y}_{t}^{0, \delta}-Y_{t}^{0, \delta}\right| \leq C \delta^{\frac{3}{2}}, \delta \in\left(0, \delta_{0}\right), P \text {-a.s. }
$$

Now we give the proof of Theorem 4.2 .

Proof. (1) Let us show that $\mathbf{W}$ is a viscosity supersolution. For this we suppose that $i \in \mathbf{K}, \phi \in C_{l, b}^{3}([0, T] \times \mathbb{R})$ and $(t, x) \in[0, T) \times \mathbb{R}$ are such that $W_{i}-\phi$ attains its minimum at $(t, x)$. Without loss of generality we may also suppose that $\phi(t, x)=W_{i}(t, x)$. Then

$$
\begin{aligned}
& \phi\left(\tau^{\delta}, X_{\tau^{\delta}}^{u, v}\right) \mathbf{1}_{\left\{N_{\tau^{\delta}}^{t, i}=i\right\}}+\sum_{l=1}^{k-1} W_{(l+i) \bmod (k)}\left(\tau^{\delta}, X_{\tau^{\delta}}^{u, v}\right) \mathbf{1}_{\left\{N_{\tau^{\delta}}^{t, i}=(l+i) \bmod (k)\right\}} \\
\leq & W_{i}\left(\tau^{\delta}, X_{\tau^{\delta}}^{u, v}\right) \mathbf{1}_{\left\{N_{\tau^{\delta}}^{t, i}=i\right\}}+\sum_{l=1}^{k-1} W_{(l+i) \bmod (k)}\left(\tau^{\delta}, X_{\tau^{\delta}}^{u, v}\right) \mathbf{1}_{\left\{N_{\tau^{\delta}}^{t, i}=(l+i) \bmod (k)\right\}} \\
= & W_{N_{\tau^{\delta}}^{t, i}}\left(\tau^{\delta}, X_{\tau^{\delta}}^{u, v}\right),(u, v) \in \mathcal{U}_{t, \tau^{\delta}} \times \mathcal{V}_{t, \tau^{\delta}}
\end{aligned}
$$

Recall from (4.4) that $\tilde{Y}_{t}^{1, i, u, v}=\phi(t, x)$, and recall also Lemma 4.3. Then, from the comparison theorem (the proof is similar to Theorem 3.3 in [6]) for fully coupled FBSDEs with jumps we have, for all $\delta \in\left(0, \delta_{0}\right)$ and for all $u \in \mathcal{U}_{t, \tau^{\delta}}, \beta \in \mathcal{B}_{t, \tau^{\delta}}$,

$$
\begin{aligned}
& Y_{t}^{1, i, u, \beta(u)}=Y_{t}^{u, \beta(u)}-\phi(t, x) \\
= & G_{t, \tau^{\delta}}^{t, x, i u, \beta(u)}\left[\phi\left(\tau^{\delta}, X_{\tau^{\delta}}^{u, \beta(u)}\right) \mathbf{1}_{\left\{N_{\tau^{\delta}}^{t, i}=i\right\}}+\sum_{l=1}^{k-1} W_{(l+i) \bmod (k)}\left(\tau^{\delta}, X_{\tau^{\delta}}^{u, \beta(u)}\right) \mathbf{1}_{\left\{N_{\tau^{\delta}}^{t, i}=(l+i) \bmod (k)\right\}}\right]-\phi(t, x) \\
\leq & G_{t, \tau^{\delta}}^{t, x, i ; u, \beta(u)}\left[W_{N_{\tau^{\delta}}^{t, i}}\left(\tau^{\delta}, \bar{X}_{\tau^{\delta}}^{t, x, i ; u, \beta(u)}\right)\right]-W_{i}(t, x),
\end{aligned}
$$

where $G_{t, \tau^{\delta}}^{t, x, i ; u, \beta(u)}[\cdot]$ is defined in (3.9), and $\left(\bar{X}^{t, x, i ; u, \beta(u)}, \bar{Y}^{t, x, i ; u, \beta(u)}, \bar{H}^{t, x, i ; u, \beta(u)}, \bar{Z}^{t, x, i ; u, \beta(u)}\right)$ is the unique solution of the following FBSDE with jumps on $\left[t, \tau^{\delta}\right]$ :

$$
\left\{\begin{aligned}
d \bar{X}_{s}^{t, x, i ; u, \beta(u)=} & b_{N_{s}^{t, i}}\left(s, \bar{X}_{s}^{t, x, i ; u, \beta(u)}, \bar{Y}_{s}^{t, x, i ; u, \beta(u)}, \bar{Z}_{s}^{t, x, i ; u, \beta(u)}, u_{s}, \beta(u)_{s}\right) \mathrm{d} s \\
& +\sigma_{N_{s}^{t, i}}\left(s, \bar{X}_{s}^{t, x, i ; u, \beta(u)}, \bar{Y}_{s}^{t, x, i ; u, \beta(u)}, u_{s}, \beta(u)_{s}\right) \mathrm{d} B_{s}, \quad s \in\left[t, \tau^{\delta}\right], \\
\bar{X}_{t}^{t, x, i ; u, \beta(u)}= & x, \\
d \bar{Y}_{s}^{t, x, i ; u, \beta(u)=} & -\tilde{f}_{N_{s}^{t, i}}\left(s, \bar{X}_{s}^{t, x, i ; u, \beta(u)}, \bar{Y}_{s}^{t, x, i ; u, \beta(u)}, \bar{H}_{s}^{t, x, i ; u, \beta(u)}, \bar{Z}_{s}^{t, x, i ; u, \beta(u)}, u_{s}, \beta(u)_{s}\right) \mathrm{d} s \\
& +\lambda \sum_{l=1}^{k-1} \bar{H}_{s}^{t, x, i ; u, \beta(u)}(l) \mathrm{d} s+\bar{Z}_{s}^{t, x, i ; u, \beta(u)} \mathrm{d} B_{s}+\sum_{l=1}^{k-1} \bar{H}_{s}^{t, x, i ; u, \beta(u)}(l) d \tilde{N}_{s}(l), \\
\bar{Y}_{\tau^{\delta}}^{t, x, i ; u, \beta(u)}= & W_{N_{\tau^{\delta}}^{t, i}}\left(\tau^{\delta}, \bar{X}_{\tau^{\delta}}^{t, x, i ; u, \beta(u)}\right) .
\end{aligned}\right.
$$

Combined with the strong DPP (Thm. 3.17), this yields

$$
\underset{\beta \in \mathcal{B}_{t, \tau^{\delta}}}{\operatorname{essinf}} \operatorname{esssup} Y_{t, \tau^{\delta}} Y_{t}^{1, i, u, \beta(u)} \leq \underset{\beta \in \mathcal{B}_{t, \tau^{\delta}}}{\operatorname{essinf}} \operatorname{esssup}_{u \in \mathcal{U}^{\delta}} G_{t, \tau^{\delta}}^{t, x, i ; u, \beta(u)}\left[W_{N_{\tau^{\delta}}^{t, i}}\left(\tau^{\delta}, \bar{X}_{\tau^{\delta}}^{t, x, i ; u, \beta(u)}\right)\right]-W_{i}(t, x)=0 \text {, P-a.s. }
$$


Furthermore, Lemma 4.6 implies that

$$
\underset{\beta \in \mathcal{B}_{t, \tau^{\delta}}}{\operatorname{essinf}} \operatorname{esssup} Y_{u \in \mathcal{U}_{t, \tau^{\delta}}} Y_{t}^{2, i, u, \beta(u)} \leq \underset{\beta \in \mathcal{B}_{t, \tau^{\delta}} \quad \operatorname{essinf}}{\operatorname{esssup}} Y_{t, \tau^{\delta}} Y_{t}^{1, i, u, \beta(u)}+C \delta^{\frac{4}{3}} \leq C \delta^{\frac{4}{3}} \text {, P-a.s. }
$$

Consequently, from $\operatorname{essinf}_{v \in \mathcal{V}_{t, \tau^{\delta}}} Y_{t}^{2, i, u, v} \leq Y_{t}^{2, i, u, \beta(u)}, \beta \in \mathcal{B}_{t, \tau^{\delta}}$, we get

$$
\underset{u \in \mathcal{U}_{t, \tau^{\delta}}}{\operatorname{essup}} \operatorname{essinf} Y_{v \in \tau^{\delta}}^{2, i, u, v} \leq \underset{\beta \in \mathcal{B}_{t, \tau^{\delta}}}{\operatorname{essinf}} \operatorname{esssup} Y_{u \in \mathcal{U}_{t, \tau}} Y_{t}^{2, i, u, \beta(u)} \leq C \delta^{\frac{4}{3}}, \text { P-a.s. }
$$

On the other hand, according to Lemma 4.7, $Y_{t}^{3, i}=\underset{u \in \mathcal{U}_{t, \tau^{\delta}}}{\operatorname{esssup}} \underset{v \in \mathcal{V}_{t, \tau^{\delta}}}{\operatorname{essinf}} Y_{t}^{2, i, u, v} \leq C \delta^{\frac{4}{3}}$, P-a.s. It then follows from Remark 4.9 and Lemma 4.10,

$$
\bar{Y}_{t}^{0, \delta} \leq C \delta^{\frac{4}{3}}
$$

Therefore, from equation (4.22), $\sup _{u \in \mathcal{U}} \inf _{v \in V} F(t, x, 0,0,0, u, v)=F_{0}(t, x, 0,0,0) \leq 0$, that is,

$$
\begin{aligned}
& \frac{\partial \phi}{\partial t}(t, x) \\
& +\sup _{u \in U} \inf _{v \in V}\left\{L_{u, v}^{i} \phi(t, x)+\tilde{f}_{i}\left(t, x, \phi(t, x), \mathbf{W}_{(i+\cdot) \bmod (k)}(t, x)-\phi(t, x) \mathbf{1}, D \phi(t, x) \sigma_{i}(t, x, \phi(t, x), u, v), u, v\right)\right\} \\
& =\frac{\partial \phi}{\partial t}(t, x)+\sup _{u \in U} \inf _{v \in V}\left\{L_{u, v}^{i} \phi(t, x)+f_{i}\left(t, x, \mathbf{W}(t, x), D \phi(t, x) \sigma_{i}(t, x, \phi(t, x), u, v), u, v\right)\right\} \leq 0 .
\end{aligned}
$$

Hence, $\mathbf{W}$ is a viscosity supersolution of (4.1).

(2) We now show that $\mathbf{W}$ is a viscosity subsolution of (4.1). Suppose $i \in \mathbf{K}, \phi \in C_{l, b}^{3}([0, T] \times \mathbb{R})$ and $(t, x) \in$ $[0, T) \times \mathbb{R}$ are such that $W_{i}-\phi$ attains its maximum at $(t, x)$. Without loss of generality, we assume again that $\phi(t, x)=W_{i}(t, x)$.

For this, we only need to prove that $\sup _{u \in U} \inf _{v \in V} F(t, x, 0,0,0, u, v)=F_{0}(t, x, 0,0,0) \geq 0$. Let us suppose that this is not true. Then there exists some $\theta>0$ such that

$$
\sup _{u \in U} \inf _{v \in V} F(t, x, 0,0,0, u, v)=F_{0}(t, x, 0,0,0) \leq-\theta<0
$$

and we can find a measurable function $g: U \rightarrow V$ such that

$$
F(t, x, 0,0,0, u, g(u)) \leq-\frac{3}{4} \theta, \text { for all } u \in U
$$

Moreover, since $F(\cdot, x, 0,0,0, \cdot, \cdot)$ is uniformly continuous on $[0, T] \times U \times V$, there exists some $T-t \geq R>0$ such that

$$
F(s, x, 0,0,0, u, g(u)) \leq-\frac{1}{2} \theta, \text { for all } u \in U \text { and }|s-t| \leq R
$$


On the other hand, notice that now we have

$$
\begin{aligned}
& \phi\left(\tau^{\delta}, X_{\tau^{\delta}}^{u, \beta(u)}\right) \mathbf{1}_{\left\{N_{\tau^{\delta}}^{t, i}=i\right\}}+\sum_{l=1}^{k-1} W_{(l+i) \bmod (k)}\left(\tau^{\delta}, X_{\tau^{\delta}}^{u, \beta(u)}\right) \mathbf{1}_{\left\{N_{\tau^{\delta}}^{t, i}=(l+i) \bmod (k)\right\}} \\
\geq & W_{i}\left(\tau^{\delta}, X_{\tau^{\delta}}^{u, \beta(u)}\right) \mathbf{1}_{\left\{N_{\tau^{\delta}}^{t, i}=i\right\}}+\sum_{l=1}^{k-1} W_{(l+i) \bmod (k)}\left(\tau^{\delta}, X_{\tau^{\delta}}^{u, \beta(u)}\right) \mathbf{1}_{\left\{N_{\tau^{\delta}}^{t, i}=(l+i) \bmod (k)\right\}} \\
= & W_{N_{\tau^{\delta}}^{t, i}}\left(\tau^{\delta}, X_{\tau^{\delta}}^{u, \beta(u)}\right) .
\end{aligned}
$$

Similar to (4.27), from the comparison theorem for fully coupled FBSDEs with jumps (see, Thm. 3.3 in [6]) and Lemma 4.1 we have

$$
\begin{aligned}
& Y_{t}^{1, i, u, \beta(u)}=Y_{t}^{u, \beta(u)}-\phi(t, x) \\
= & G_{t, \tau^{\delta}}^{t, x, i ; u(u)}\left[\phi\left(\tau^{\delta}, X_{\tau^{\delta}}^{u, \beta(u)}\right) \mathbf{1}_{\left\{N_{\tau^{\delta}}^{t, i}=i\right\}}+\sum_{l=1}^{k-1} W_{(l+i) \bmod (k)}\left(\tau^{\delta}, X_{\tau^{\delta}}^{u, \beta(u)}\right) \mathbf{1}_{\left\{N_{\tau^{\delta}}^{t, i}=(l+i) \bmod (k)\right\}}\right]-\phi(t, x) \\
\geq & G_{t, \tau^{\delta}}^{t, x, i ; u, \beta(u)}\left[W_{N_{\tau^{\delta}}^{t, i}}\left(\tau^{\delta}, \bar{X}_{\tau^{\delta}}^{t, x, i ; u, \beta(u)}\right)\right]-W_{i}(t, x),
\end{aligned}
$$

where $G_{t, \tau^{\delta}}^{t, x, i ; u, \beta(u)}[\cdot]$ is defined in (3.9). Then, the strong DPP gives $\underset{\beta \in \mathcal{B}_{t, \tau} \delta}{\operatorname{essinf}} \operatorname{esssup}_{u \in \mathcal{U}_{t, \tau} \delta} Y_{t}^{1, i, u, \beta(u)} \geq 0$, P-a.s., and in particular, $\operatorname{esssup}_{u \in \mathcal{U}_{t} \delta} Y_{t}^{1, i, u, g(u)} \geq 0$, P-a.s. Here, by putting $g_{s}(u)(\omega)=g\left(u_{s}(\omega)\right),(s, \omega) \in[t, T] \times \Omega$, we identify $g$ as an element of $\mathcal{B}_{t, \tau^{\delta}}$. Given an arbitrary $\varepsilon>0$ we can choose $u^{\varepsilon} \in \mathcal{U}_{t, \tau^{\delta}}$ (depending on $\delta>0$ ) such that $Y_{t}^{1, i, u^{\varepsilon}, g\left(u^{\varepsilon}\right)} \geq-\varepsilon \delta$. From Lemma 4.6 we have

$$
Y_{t}^{2, i, u^{\varepsilon}, g\left(u^{\varepsilon}\right)} \geq-C \delta^{\frac{4}{3}}-\varepsilon \delta, \text { P-a.s. }
$$

Recall that

$$
\begin{aligned}
Y_{t}^{2, i, u^{\varepsilon}, g\left(u^{\varepsilon}\right)=} & \int_{t}^{\tau^{\delta}} F\left(s, x, 0, H_{s}^{2, i, u^{\varepsilon}, g\left(u^{\varepsilon}\right)}, Z_{s}^{2, i, u^{\varepsilon}, g\left(u^{\varepsilon}\right)}, u_{s}^{\varepsilon}, g\left(u_{s}^{\varepsilon}\right)\right) \mathrm{d} s-\int_{t}^{\tau^{\delta}} \lambda \sum_{l=1}^{k-1} H_{s}^{2, i, u^{\varepsilon}, g\left(u^{\varepsilon}\right)}(l) \mathrm{d} s \\
& -\int_{t}^{\tau^{\delta}} Z_{s}^{2, i, u^{\varepsilon}, g\left(u^{\varepsilon}\right)} \mathrm{d} B_{s}-\int_{t}^{\tau^{\delta}} \sum_{l=1}^{k-1} H_{s}^{2, i, u^{\varepsilon}, g\left(u^{\varepsilon}\right)}(l) d \tilde{N}_{s}(l) .
\end{aligned}
$$

Using that $(h, z) \rightarrow F(s, x, 0, h, z, u, v)$ is Lipschitz, uniformly in $(s, x, u, v)$, we have

$$
\begin{aligned}
& F\left(s, x, 0, H_{s}^{2, i, u^{\varepsilon}, g\left(u^{\varepsilon}\right)}, Z_{s}^{2, i, u^{\varepsilon}, g\left(u^{\varepsilon}\right)}, u_{s}^{\varepsilon}, g\left(u_{s}^{\varepsilon}\right)\right) \\
\leq & F\left(s, x, 0,0,0, u_{s}^{\varepsilon}, g\left(u_{s}^{\varepsilon}\right)\right)+C\left(\sum_{l=1}^{k-1}\left|H_{s}^{2, i, u^{\varepsilon}, g\left(u^{\varepsilon}\right)}(l)\right|+\left|Z_{s}^{2, i, u^{\varepsilon}, g\left(u^{\varepsilon}\right)}\right|\right) .
\end{aligned}
$$

Hence,

$$
\begin{aligned}
& Y_{t}^{2, i, u^{\varepsilon}, g\left(u^{\varepsilon}\right)} \leq \mathbb{E}\left[\int_{t}^{\tau^{\delta}} F\left(s, x, 0,0,0, u_{s}^{\varepsilon}, g\left(u_{s}^{\varepsilon}\right)\right) \mathrm{d} s \mid \mathcal{F}_{t}\right] \\
& +C \mathbb{E}\left[\int_{t}^{\tau^{\delta}}\left(\lambda \sum_{l=1}^{k-1}\left|H_{s}^{2, i, u^{\varepsilon}, g\left(u^{\varepsilon}\right)}(l)\right|+\left|Z_{s}^{2, i, u^{\varepsilon}, g\left(u^{\varepsilon}\right)}\right|\right) \mathrm{d} s \mid \mathcal{F}_{t}\right] .
\end{aligned}
$$


Notice that, from (4.8) and (4.14)-(ii) we have

$$
\begin{aligned}
& \mathbb{E}\left[\int_{t}^{\tau^{\delta}}\left(\lambda \sum_{l=1}^{k-1}\left|H_{s}^{2, i, u^{\varepsilon}, g\left(u^{\varepsilon}\right)}(l)\right|+\left|Z_{s}^{2, i, u^{\varepsilon}, g\left(u^{\varepsilon}\right)}\right|\right) \mathrm{d} s \mid \mathcal{F}_{t}\right] \\
\leq & \mathbb{E}\left[\int_{t}^{\tau^{\delta}}\left(\lambda \sum_{l=1}^{k-1}\left|H_{s}^{1, i, u^{\varepsilon}, g\left(u^{\varepsilon}\right)}(l)\right|+\left|Z_{s}^{1, i, u^{\varepsilon}, g\left(u^{\varepsilon}\right)}\right|\right) \mathrm{d} s \mid \mathcal{F}_{t}\right] \\
& +\mathbb{E}\left[\int_{t}^{\tau^{\delta}}\left(\lambda \sum_{l=1}^{k-1}\left|H_{s}^{1, i, u^{\varepsilon}, g\left(u^{\varepsilon}\right)}(l)-H_{s}^{2, i, u^{\varepsilon}, g\left(u^{\varepsilon}\right)}(l)\right|+\left|Z_{s}^{1, i, u^{\varepsilon}, g\left(u^{\varepsilon}\right)}-Z_{s}^{2, i, u^{\varepsilon}, g\left(u^{\varepsilon}\right)}\right|\right) \mathrm{d} s \mid \mathcal{F}_{t}\right] \\
\leq & C \delta^{\frac{5}{4}}+C \delta^{\frac{1}{2}}\left(\mathbb{E}\left[\int_{t}^{\tau^{\delta}}\left(\lambda \sum_{l=1}^{k-1}\left|H_{s}^{1, i, u^{\varepsilon}, g\left(u^{\varepsilon}\right)}(l)-H_{s}^{2, i, u^{\varepsilon}, g\left(u^{\varepsilon}\right)}(l)\right|^{2}+\left|Z_{s}^{1, i, u^{\varepsilon}, g\left(u^{\varepsilon}\right)}-Z_{s}^{2, i, u^{\varepsilon}, g\left(u^{\varepsilon}\right)}\right|^{2}\right) \mathrm{d} s \mid \mathcal{F}_{t}\right]\right)^{\frac{1}{2}} \\
\leq & C \delta^{\frac{5}{4}}+C \delta^{\frac{4}{3}} \leq C \delta^{\frac{5}{4}} .
\end{aligned}
$$

Substituting this in (4.31) yields

$$
Y_{t}^{2, i, u^{\varepsilon}, g\left(u^{\varepsilon}\right)} \leq \mathbb{E}\left[\int_{t}^{\tau^{\delta}} F\left(s, x, 0,0,0, u_{s}^{\varepsilon}, g\left(u_{s}^{\varepsilon}\right)\right) \mathrm{d} s \mid \mathcal{F}_{t}\right]+C \delta^{\frac{5}{4}} .
$$

Consequently, from (4.29), for all $\delta \in\left(0, \delta_{0} \wedge R\right)$,

$$
Y_{t}^{2, i, u^{\varepsilon}, g\left(u^{\varepsilon}\right)} \leq-\frac{1}{2} \theta \mathbb{E}\left[\tau^{\delta}-t \mid \mathcal{F}_{t}\right]+C \delta^{\frac{5}{4}}
$$

From the definition of $\tau^{\delta}, \tau^{\delta}=t+\delta$ on $\left\{N_{t+\delta}^{t, i}=i\right\}$. Thus,

$$
\begin{aligned}
& \mathbb{E}\left[\tau^{\delta} \mid \mathcal{F}_{t}\right] \geq(t+\delta) P\left\{N_{t+\delta}^{t, i}=i \mid \mathcal{F}_{t}\right\}=(t+\delta) P\left\{N_{t+\delta}^{t, i}=i\right\} \\
& =(t+\delta) P\{N((t, t+\delta] \times\{l\})=0,1 \leq l \leq k-1\} \\
& =(t+\delta) e^{-\lambda(k-1) \delta}=: \psi(\delta) .
\end{aligned}
$$

As $\psi^{\prime}(\delta)=e^{-\lambda(k-1) \delta}(1-\lambda(k-1)(t+\delta)) \geq e^{-\lambda(k-1) \delta}(1-\lambda(k-1) T)>0$, the function $\psi:[0, T] \rightarrow \mathbb{R}$ is increasing, and there exists some $\hat{\delta} \in[0, \delta]$, such that $\psi(\delta)-\psi(0)=\psi^{\prime}(\hat{\delta}) \delta$, i.e.,

$$
\mathbb{E}\left[\tau^{\delta}-t \mid \mathcal{F}_{t}\right] \geq \psi(\delta)-\psi(0)=\psi^{\prime}(\hat{\delta}) \delta=e^{-\lambda(k-1) \hat{\delta}}(1-\lambda(k-1)(t+\hat{\delta})) \delta \geq e^{-\lambda(k-1) \delta}(1-\lambda(k-1) T) \delta(>0) .
$$

Hence, from (4.33),

$$
Y_{t}^{2, i, u^{\varepsilon}, g\left(u^{\varepsilon}\right)} \leq-\frac{1}{2} \theta \mathbb{E}\left[\tau^{\delta}-t \mid \mathcal{F}_{t}\right]+C \delta^{\frac{5}{4}} \leq-\frac{1}{2} \theta e^{-\lambda(k-1) \delta}(1-\lambda(k-1) T) \delta+C \delta^{\frac{5}{4}} .
$$

From (4.30) and (4.34), $-C \delta^{\frac{1}{3}}-\varepsilon \leq-\frac{1}{2} \theta e^{-\lambda(k-1) \delta}(1-\lambda(k-1) T)+C \delta^{\frac{1}{4}}$, P-a.s. Letting $\delta \downarrow 0$, and then $\varepsilon \downarrow 0$, we deduce that $\theta \leq 0$, which induces a contradiction. Therefore,

$$
\sup _{u \in \mathcal{U}} \inf _{v \in V} F(t, x, 0,0,0, u, v)=F_{0}(t, x, 0,0,0) \geq 0
$$

and from the definition of $F$ we see that $\mathbf{W}$ is a viscosity subsolution of (4.1).

Therefore, we obtain $\mathbf{W}$ is a viscosity solution of (4.1). 


\subsection{Uniqueness}

First we define the following space of continuous functions:

$$
\begin{gathered}
\Theta=\left\{\varphi \in C([0, T] \times \mathbb{R}): \exists \tilde{A}>0 \text { such that } \lim _{|x| \rightarrow \infty} \varphi(t, x) \exp \left\{-\tilde{A}\left[\log \left(\left(|x|^{2}+1\right)^{\frac{1}{2}}\right)\right]^{2}\right\}=0,\right. \\
\text { uniformly in } t \in[0, T]\} .
\end{gathered}
$$

The uniqueness result of viscosity solution of the following HJBI equation (which is a special case of (4.1) when the diffusion coefficients $\sigma_{i}, i \in \mathbf{K}$, are independent of $y$ ) will be presented in $\Theta$ :

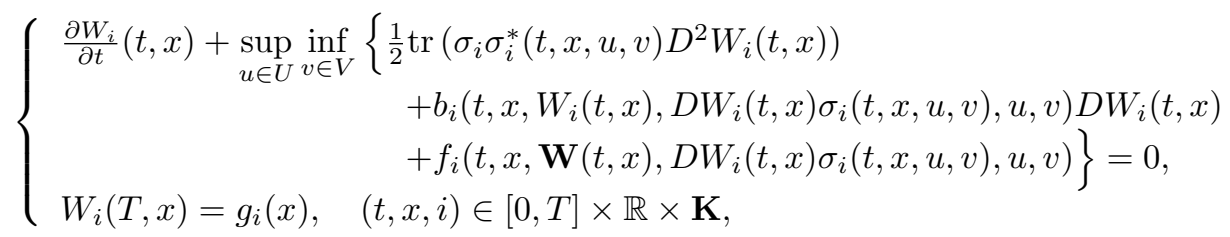

where $\mathbf{W}(t, x)=\left(W_{1}(t, x), W_{2}(t, x), \cdots, W_{k}(t, x)\right),(t, x) \in[0, T] \times \mathbb{R}$.

Theorem 4.11. Under the assumptions (B1)-(B3), the lower value function $\mathbf{W}$ is the unique viscosity solution in $\Theta$ of the coupled HJBI equation (4.35).

Proving the uniqueness of the viscosity solution similar techniques as those in [7] can be applied in our case. So the details are omitted here. We only study the lower value function $\mathbf{W}(t, x)$. For the upper value function $\mathbf{U}(t, x)=\left(U_{1}(t, x), U_{2}(t, x), \cdots, U_{k}(t, x)\right)$, we have the same result.

Theorem 4.12. Under our assumptions (B1)-(B3), the upper value function $\mathbf{U}(t, x)=\left(U_{1}(t, x), U_{2}(t, x)\right.$, $\left.\cdots, U_{k}(t, x)\right)$ is a viscosity solution of the following system of HJBI equations:

$$
\left\{\begin{aligned}
& \frac{\partial U_{i}}{\partial t}(t, x)+\inf _{v \in V} \sup _{u \in U}\{ b_{i}\left(t, x, U_{i}(t, x), D U_{i}(t, x) \sigma_{i}\left(t, x, U_{i}(t, x), u, v\right), u, v\right) D U_{i}(t, x) \\
&+\frac{1}{2} \operatorname{tr}\left(\sigma_{i} \sigma_{i}^{*}\left(t, x, U_{i}(t, x), u, v\right) D^{2} U_{i}(t, x)\right) \\
&\left.+f_{i}\left(t, x, \mathbf{U}(t, x), D U_{i}(t, x) \sigma_{i}\left(t, x, U_{i}(t, x), u, v\right), u, v\right)\right\}=0, \\
& U_{i}(T, x)=g_{i}(x),(t, x) \in[0, T] \times \mathbb{R}, i \in \mathbf{K}=\{1,2, \cdots k\} .
\end{aligned}\right.
$$

When $\sigma_{i}, i \in \mathbf{K}$, do not depend on $y$, we also get the uniqueness of the viscosity solution $\mathbf{U}(t, x)$ in $\Theta$.

Remark 4.13. If for all $(t, x, i) \in[0, T] \times \mathbb{R} \times \mathbf{K},(y, p, A) \in \mathbb{R} \times \mathbb{R} \times \mathbb{R}, a \in \mathbb{R}^{k}$, the following Isaacs' condition holds true:

$$
\begin{aligned}
& \sup _{u \in U} \inf _{v \in V}\left\{\frac{1}{2} \operatorname{tr}\left(\sigma_{i} \sigma_{i}^{*}(t, x, u, v) A\right)+b_{i}\left(t, x, y, p \sigma_{i}(t, x, u, v), u, v\right) \cdot p+f_{i}\left(t, x, a, p \sigma_{i}(t, x, u, v), u, v\right)\right\} \\
= & \inf _{v \in V} \sup _{u \in U}\left\{\frac{1}{2} \operatorname{tr}\left(\sigma_{i} \sigma_{i}^{*}(t, x, u, v) A\right)+b_{i}\left(t, x, y, p \sigma_{i}(t, x, u, v), u, v\right) \cdot p+f_{i}\left(t, x, a, p \sigma_{i}(t, x, u, v), u, v\right)\right\},
\end{aligned}
$$

then the equations (4.35) and (4.36) coincide (when $\sigma_{i}, i \in \mathbf{K}$, do not depend on $y$ ). The uniqueness of the viscosity solution implies that $\mathbf{W}(t, x)=\mathbf{U}(t, x)$. That is, there exists a value for our stochastic differential games under the Isaacs' condition. 


\section{APPENDIX A.}

\section{A.1 Comparison theorem for FBSDEs with jumps}

In this section, we show that the comparison theorem for the dynamics (3.2) holds true only when $K_{1} \geq 0$, where $K_{1}$ is the constant in the condition (B3). For simplicity, we suppress the controls $(u, v)$ in equation (3.2), i.e., the following FBSDE with jumps,

$$
\left\{\begin{aligned}
\mathrm{d} X_{s}^{t, \eta, i}= & b_{N_{s}^{t, i}}\left(s, X_{s}^{t, \eta, i}, Y_{s}^{t, \eta, i}, Z_{s}^{t, \eta, i}\right) \mathrm{d} s+\sigma_{N_{s}^{t, i}}\left(s, X_{s}^{t, \eta, i}, Y_{s}^{t, \eta, i}\right) \mathrm{d} B_{s}, \quad s \in[t, T], \\
X_{t}^{t, \eta, i}= & \eta, \\
\mathrm{d} Y_{s}^{t, \eta, i}= & -\left(\tilde{f}_{N_{s}^{t, i}}\left(s, X_{s}^{t, \eta, i}, Y_{s}^{t, \eta, i}, H_{s}^{t, \eta, i}, Z_{s}^{t, \eta, i}\right)-\lambda \sum_{l=1}^{k-1} H_{s}^{t, \eta, i}(l)\right) \mathrm{d} s \\
& +Z_{s}^{t, \eta, i} \mathrm{~d} B_{s}+\sum_{l=1}^{k-1} H_{s}^{t, \eta, i}(l) \mathrm{d} \tilde{N}_{s}(l), \quad s \in[t, T] \\
Y_{T}^{t, \eta, i}= & g_{N_{T}^{t, i}}\left(X_{T}^{t, \eta, i}\right),
\end{aligned}\right.
$$

where $i \in \mathbf{K}$, and initial data $(t, \eta) \in[0, T] \times L^{2}\left(\Omega, \mathcal{F}_{t}, P ; \mathbb{R}\right)$. From Remark 3.3, we know that, under the assumptions (B1) and (B2), equation (A.1) has a unique solution $\left(X^{t, \eta, i}, Y^{t, \eta, i}, H^{t, \eta, i}, Z^{t, \eta, i}\right) \in \mathcal{B}^{2}[t, T]$. We now show that under the additional condition (B3) the comparison theorem for this equation holds true (or, equivalently, if condition (iii) in Rem. 3.1 is satisfied).

In fact, when the constant $K_{1}>0$ we get the comparison theorem by arguments similar to those in Theorem 2.5 in [11]. For the situation $K_{1}=0$, we consider an increasing positive sequence $\left\{\lambda_{n}\right\}_{n=1}^{\infty} \subset \mathbb{R}_{+}$with $\lambda_{n}<\lambda$, $\lambda_{n} \uparrow \lambda, n \rightarrow \infty$, and the FBSDE with jumps (A.1) with $\lambda$ replaced by $\lambda_{n}$ (but we keep $\tilde{N}_{s}(l)$ defined in Subsection 2.1 with $\lambda$ ), that is,

$$
\left\{\begin{aligned}
\mathrm{d} X_{s}^{t, \eta, i, n}= & b_{N_{s}^{t, i}}\left(s, X_{s}^{t, \eta, i, n}, Y_{s}^{t, \eta, i, n}, Z_{s}^{t, \eta, i, n}\right) \mathrm{d} s+\sigma_{N_{s}^{t, i}}\left(s, X_{s}^{t, \eta, i, n}, Y_{s}^{t, \eta, i, n}\right) \mathrm{d} B_{s}, \quad s \in[t, T], \\
X_{t}^{t, \eta, i, n}= & \eta, \\
\mathrm{d} Y_{s}^{t, \eta, i, n}= & -\left(\tilde{f}_{N_{s}^{t, i}}\left(s, X_{s}^{t, \eta, i, n}, Y_{s}^{t, \eta, i, n}, H_{s}^{t, \eta, i, n}, Z_{s}^{t, \eta, i, n}\right)-\lambda_{n} \sum_{l=1}^{k-1} H_{s}^{t, \eta, i, n}(l)\right) \mathrm{d} s \\
& +Z_{s}^{t, \eta, i, n} \mathrm{~d} B_{s}+\sum_{l=1}^{k-1} H_{s}^{t, \eta, i, n}(l) \mathrm{d} \tilde{N}_{s}(l), \quad s \in[t, T], \\
Y_{T}^{t, \eta, i, n}= & g_{N_{T}^{t, i}}\left(X_{T}^{t, \eta, i, n}\right) .
\end{aligned}\right.
$$

It is easy to check that, for every $n \in \mathbb{N}$, this equation has a unique solution $\left(X^{t, \eta, i, n}, Y^{t, \eta, i, n}, H^{t, \eta, i, n}, Z^{t, \eta, i, n}\right) \in$ $\mathcal{B}^{2}[t, T]$. For $n \in \mathbb{N}$ and $i \in \mathbf{K}$, we introduce

$$
\tilde{f}_{i}^{n}(s, x, y, h, z)=\tilde{f}_{i}(s, x, y, h, z)+\left(\lambda-\lambda_{n}\right) \sum_{l=1}^{k-1} h(l), \quad(s, x, y, z, h) \in[t, T] \times \mathbb{R} \times \mathbb{R} \times \mathbb{R}^{k-1} \times \mathbb{R}^{d} .
$$

Then the equation (A.2) can be rewritten as

$$
\left\{\begin{aligned}
\mathrm{d} X_{s}^{t, \eta, i, n}= & b_{N_{s}^{t, i}}\left(s, X_{s}^{t, \eta, i, n}, Y_{s}^{t, \eta, i, n}, Z_{s}^{t, \eta, i, n}\right) \mathrm{d} s+\sigma_{N_{s}^{t, i}}\left(s, X_{s}^{t, \eta, i, n}, Y_{s}^{t, \eta, i, n}\right) \mathrm{d} B_{s}, \quad s \in[t, T], \\
X_{t}^{t, \eta, i, n}= & \eta, \\
\mathrm{d} Y_{s}^{t, \eta, i, n}= & -\left(\tilde{f}_{N_{s}^{t, i}}^{n}\left(s, X_{s}^{t, \eta, i, n}, Y_{s}^{t, \eta, i, n}, H_{s}^{t, \eta, i, n}, Z_{s}^{t, \eta, i, n}\right)-\lambda \sum_{l=1}^{k-1} H_{s}^{t, \eta, i, n}(l)\right) \mathrm{d} s \\
& +Z_{s}^{t, \eta, i, n} \mathrm{~d} B_{s}+\sum_{l=1}^{k-1} H_{s}^{t, \eta, i, n}(l) \mathrm{d} \tilde{N}_{s}(l), \quad s \in[t, T], \\
Y_{T}^{t, \eta, i, n}= & g_{N_{T}^{t, i}}\left(X_{T}^{t, \eta, i, n}\right) .
\end{aligned}\right.
$$


Since the coefficient $\tilde{f}_{i}^{n}(s, x, y, h, z)$ satisfies the condition (iii) in Remark 3.1 with $K_{1}+\left(\lambda-\lambda_{n}\right)>K_{1}=0$, we get the comparison results for the FBSDE with jumps (A.3) for all $n \in \mathbb{N}$. Note that it follows from standard arguments that

$$
\begin{aligned}
& E\left[\sup _{s \in[t, T]}\left|X_{s}^{t, \eta, i, n}-X_{s}^{t, \eta, i}\right|^{2}+\sup _{s \in[t, T]}\left|Y_{s}^{t, \eta, i, n}-Y_{s}^{t, \eta, i}\right|^{2}+\int_{t}^{T}\left|Z_{s}^{t, \eta, i, n}-Z_{s}^{t, \eta, i}\right|^{2} d s\right. \\
& \left.+\lambda \sum_{l=1}^{k-1} \int_{t}^{T}\left|H_{s}^{t, \eta, i, n}(l)-H_{s}^{t, \eta, i}(l)\right|^{2} d s\right] \rightarrow 0, \text { as } n \rightarrow \infty .
\end{aligned}
$$

Thus, also for the limit equation (A.1) with $K_{1}=0$, we have the comparison theorem. This proves that we obtain the comparison theorem for FBSDE with jumps (A.1) when the constant $K_{1} \geq 0$.

It is worth to point out that, in general, there is no comparison result for FBSDEs with jumps of the type (A.1) when $K_{1}<0$. Let us give a counter-example.

Example A.1. We choose $\mathbf{K}=\{1,2\}$, i.e., $k=2$. Referring to the notations introduced in Subsection 2.1, we put

$$
N((s, t]):=N((s, t] \times\{1\}), \tilde{N}((s, t]):=(N-\hat{N})((s, t] \times\{1\}), 0 \leq s \leq t \leq T .
$$

Let the coefficients $b=0, \sigma=0$, and $\tilde{f}_{i}(s, x, y, h, z)=-\gamma \sum_{l=1}^{k-1} h(l)=-\gamma h(1)$, for some $\gamma>0$. Moreover, for $K_{1}=-\gamma<0$, we have

$$
\tilde{f}_{i}(s, x, y, h, z)-\tilde{f}_{i}\left(s, x, y, h^{\prime}, z\right)=K_{1}\left(h(1)-h^{\prime}(1)\right)
$$

but as $K_{1}<0$, (B3) or condition (iii) in Remark 3.1 is not satisfied. Notice that now (A.1) takes the form

$$
\mathrm{d} Y_{s}=(\gamma+\lambda) H_{s} \mathrm{~d} s+H_{s} \mathrm{~d} \tilde{N}_{s}=\gamma H_{s} \mathrm{~d} s+H_{s} d N_{s}, \quad s \in[0, T] .
$$

Endowed with a terminal condition $\xi \in L^{2}(\Omega, \sigma\{N((0, T])\}, P)$, BSDE (A.4) has a unique square integrable adapted solution $(Y, H)$.

Let us consider the both terminal conditions $\xi^{1}=0, \xi^{2}=N((0, T])$, and denote by $\left(Y^{i}, H^{i}\right)$ the unique solution of (A.4) with $Y_{T}^{i}=\xi^{i}, i=1,2$, respectively. Obviously,

$$
\begin{aligned}
& \left(Y_{s}^{1}, H_{s}^{1}\right) \equiv(0,0), \\
& \left(Y_{s}^{2}, H_{s}^{2}\right)=(N((0, s])-\gamma(T-s), 1), s \in[0, T] .
\end{aligned}
$$

We observe that, although $\xi^{1} \leq \xi^{2}$ with $P\left\{\xi^{2}>\xi^{1}\right\}>0$, we have $Y_{0}^{2}=-\gamma T<0=Y_{0}^{1}$, for any $\gamma>0$ (and, hence, for any $\left.K_{1}<0\right)$. This shows that, if $K_{1}<0$, the comparison theorem fails.

If we consider $\eta^{i}=i+N((0, T]), i=1,2$, and denote by $\left(\bar{Y}^{i}, \bar{H}^{i}\right)$ the unique solution of (A.4) with $\bar{Y}_{T}^{i}=\eta^{i}$, $i=1,2$, respectively. It is easy to check that, for $i=1,2$,

$$
\left(\bar{Y}_{s}^{i}, \bar{H}_{s}^{i}\right)=(i+N((0, s])-\gamma(T-s), 1), s \in[0, T] .
$$

Then, although $\xi^{1}<\eta^{i}, i=1,2$,

$$
\bar{Y}_{0}^{i}=i-\gamma T \leq 2-\gamma T<0=Y_{0}^{1}, \quad \text { if } T>\frac{2}{\gamma},
$$


which also implies that the comparison result fails for $K_{1}=-\gamma<-\frac{2}{T}$. We see, in particular that, choosing $T>0$ large enough, we can have $K_{1}<0$ arbitrarily near to zero.

Remark A.2. We consider $\eta^{i}$ in order to get the explicit solution, instead of considering the terminal condition $\bar{Y}_{T}^{i}=N_{T}^{i}=(i+N((0, T])) \bmod (2) \in\{1,2\}$. But, as $i+N((0, T]) \geq(i+N((0, T])) \bmod (2)>0$, the comparison theorem should also hold for $i+N((0, T])$ if we had the comparison theorem.

\section{A.2 The proof of Theorem 3.17 (Strong-DPP)}

For convenience, we set

$$
\bar{W}_{i}(t, x)=\underset{\beta \in \mathcal{B}_{t, \tau}}{\operatorname{essinf}} \operatorname{essuup} G_{t, \tau}^{t, x, i ; u, \beta(u)}\left[W_{N_{\tau}^{t, i}}\left(\tau, \bar{X}_{\tau}^{t, x, i ; u, \beta(u)}\right)\right] .
$$

We want to prove that $\bar{W}_{i}(t, x)$ and $W_{i}(t, x)$ coincide. For this we only need to prove the following three lemmas.

Lemma A.3. For all $i \in \mathbf{K},(t, x) \in[0, T] \times \mathbb{R}, \bar{W}_{i}(t, x)$ is deterministic.

The proof of this lemma is similar to the proof of Proposition 3.6; so we omit it here.

Lemma A.4. For all $i \in \mathbf{K},(t, x) \in[0, T] \times \mathbb{R}$, it holds $\bar{W}_{i}(t, x) \leq W_{i}(t, x), P$-a.s.

Proof. Let $\beta \in \mathcal{B}_{t, T}$ be arbitrarily fixed. For any given $u_{2} \in \mathcal{U}_{\tau, T}$, we define, for $u_{1} \in \mathcal{U}_{t, \tau}$,

$$
\beta_{1}\left(u_{1}\right):=\left.\beta\left(u_{1} \oplus u_{2}\right)\right|_{[t, \tau]},
$$

where $u_{1} \oplus u_{2}:=u_{1} \mathbf{1}_{[t, \tau]}+u_{2} \mathbf{1}_{(\tau, T]}$, belongs to $\mathcal{U}_{t, T}$. It is easy to check that $\beta_{1} \in \mathcal{B}_{t, \tau}$ and $\beta_{1}$ is independent of the special choice of $u_{2} \in \mathcal{U}_{\tau, T}$ due to the nonanticipativity property of $\beta$. Consequently, from the definition of $\bar{W}_{i}(t, x)$, Lemma 5.1 and Remark 3.7 in [7], we know for any $\varepsilon>0$, there exists $u_{1}^{\varepsilon} \in \mathcal{U}_{t, \tau}$ such that

$$
\bar{W}_{i}(t, x) \leq G_{t, \tau}^{t, x, i ; u_{1}^{\varepsilon}, \beta_{1}\left(u_{1}^{\varepsilon}\right)}\left[W_{N_{\tau}^{t, i}}\left(\tau, \bar{X}_{\tau}^{t, x, i ; u_{1}^{\varepsilon}, \beta_{1}\left(u_{1}^{\varepsilon}\right)}\right)\right]+\varepsilon, \text { P-a.s. }
$$

We now estimate $W_{N_{\tau}^{t, i}}\left(\tau, \bar{X}_{\tau}^{t, x, i ; u_{1}^{\varepsilon}, \beta_{1}\left(u_{1}^{\varepsilon}\right)}\right)$. For $u_{2} \in \mathcal{U}_{\tau, T}$, we define

$$
\beta_{2}^{u_{1}^{\varepsilon}}\left(u_{2}\right):=\left.\beta\left(u_{1}^{\varepsilon} \oplus u_{2}\right)\right|_{[\tau, T]} .
$$

Then $\beta_{2}^{u_{1}^{\varepsilon}} \in \mathcal{B}_{\tau, T}$. From Proposition 3.16 with choosing $\eta=\bar{X}_{\tau}^{t, x, i ; u_{1}^{\varepsilon}, \beta_{1}\left(u_{1}^{\varepsilon}\right)}$, we have

$$
\begin{aligned}
& W_{N_{\tau}^{t, i}}\left(\tau, \bar{X}_{\tau}^{t, x, i ; u_{1}^{\varepsilon}, \beta_{1}\left(u_{1}^{\varepsilon}\right)}\right)=\operatorname{essinf}_{\beta_{2} \in \mathcal{B}_{\tau, T}} \operatorname{esssup}_{u_{2} \in \mathcal{U}_{\tau, T}} J\left(\tau, \bar{X}_{\tau}^{t, x, i ; u_{1}^{\varepsilon}, \beta_{1}\left(u_{1}^{\varepsilon}\right)}, N_{\tau}^{t, i} ; u_{2}, \beta_{2}\left(u_{2}\right)\right) \\
& \leq \operatorname{esssup}_{u_{2} \in \mathcal{U}_{\tau, T}} J\left(\tau, \bar{X}_{\tau}^{t, x, i ; u_{1}^{\varepsilon}, \beta_{1}\left(u_{1}^{\varepsilon}\right)}, N_{\tau}^{t, i} ; u_{2}, \beta_{2}^{u_{1}^{\varepsilon}}\left(u_{2}\right)\right), P \text {-a.s. }
\end{aligned}
$$

By similar arguments to (A.5) it follows that there exists $u_{2}^{\varepsilon} \in \mathcal{U}_{\tau, T}$ such that

$$
W_{N_{\tau}^{t, i}}\left(\tau, \bar{X}_{\tau}^{t, x, i ; u_{1}^{\varepsilon}, \beta_{1}\left(u_{1}^{\varepsilon}\right)}\right) \leq J\left(\tau, \bar{X}_{\tau}^{t, x, i ; u_{1}^{\varepsilon}, \beta_{1}\left(u_{1}^{\varepsilon}\right)}, N_{\tau}^{t, i} ; u_{2}^{\varepsilon}, \beta_{2}^{u_{1}^{\varepsilon}}\left(u_{2}^{\varepsilon}\right)\right)+\varepsilon, P \text {-a.s. }
$$


It is easy to check that $\beta\left(u^{\varepsilon}\right)=\beta_{1}\left(u_{1}^{\varepsilon}\right) \oplus \beta_{2}^{u_{1}^{\varepsilon}}\left(u_{2}^{\varepsilon}\right)$, where $u^{\varepsilon}=u_{1}^{\varepsilon} \oplus u_{2}^{\varepsilon}$ belongs to $\mathcal{U}_{t, T}$. Then we have

$$
\begin{aligned}
W_{N_{\tau}^{t, i}}\left(\tau, \bar{X}_{\tau}^{t, x, i ; u_{1}^{\varepsilon}, \beta_{1}\left(u_{1}^{\varepsilon}\right)}\right) & \leq J\left(\tau, \bar{X}_{\tau}^{t, x, i ; u_{1}^{\varepsilon}, \beta_{1}\left(u_{1}^{\varepsilon}\right)}, N_{\tau}^{t, i} ; u_{2}^{\varepsilon}, \beta_{2}^{u_{1}^{\varepsilon}}\left(u_{2}^{\varepsilon}\right)\right)+\varepsilon \\
& =J\left(\tau, \bar{X}_{\tau}^{t, x, i ; u^{\varepsilon}, \beta\left(u^{\varepsilon}\right)}, N_{\tau}^{t, i} ; u^{\varepsilon}, \beta\left(u^{\varepsilon}\right)\right)+\varepsilon, P \text {-a.s. }
\end{aligned}
$$

Therefore, from (A.5), (A.6) and the comparison theorem for FBSDE with jumps (see Thm. 3.3 in [6]) we obtain

$$
\begin{aligned}
& \bar{W}_{i}(t, x) \leq G_{t, \tau}^{t, x, i ; u_{1}^{\varepsilon}, \beta_{1}\left(u_{1}^{\varepsilon}\right)}\left[W_{N_{\tau}^{t, i}}\left(\tau, \bar{X}_{\tau}^{t, x, i ; u_{1}^{\varepsilon}, \beta_{1}\left(u_{1}^{\varepsilon}\right)}\right)\right]+\varepsilon \\
& \leq G_{t, \tau}^{t, x, i ; u_{1}^{\varepsilon}, \beta_{1}\left(u_{1}^{\varepsilon}\right)}\left[J\left(\tau, \bar{X}_{\tau}^{t, x, i ; u^{\varepsilon}, \beta\left(u^{\varepsilon}\right)}, N_{\tau}^{t, i} ; u^{\varepsilon}, \beta^{\varepsilon}\left(u^{\varepsilon}\right)\right)+\varepsilon\right]+\varepsilon \\
& \leq G_{t, \tau}^{t, x, i ; u_{1}^{\varepsilon}, \beta_{1}\left(u_{1}^{\varepsilon}\right)}\left[J\left(\tau, X_{\tau}^{t, x, i ; u^{\varepsilon}, \beta\left(u^{\varepsilon}\right)}, N_{\tau}^{t, i} ; u^{\varepsilon}, \beta^{\varepsilon}\left(u^{\varepsilon}\right)\right)\right]+C \varepsilon, P \text {-a.s. }
\end{aligned}
$$

where the last inequality follows from Corollary 3.1 in [6]. Finally, from (A.7) we get

$$
\begin{aligned}
\bar{W}_{i}(t, x) & \leq G_{t, \tau}^{t, x, i ; u^{\varepsilon}, \beta\left(u^{\varepsilon}\right)}\left[J\left(\tau, X_{\tau}^{t, x, i ; u^{\varepsilon}, \beta\left(u^{\varepsilon}\right)}, N_{\tau}^{t, i} ; u^{\varepsilon}, \beta^{\varepsilon}\left(u^{\varepsilon}\right)\right)\right]+C \varepsilon \\
& =G_{t, \tau}^{t, x, i ; u^{\varepsilon}, \beta\left(u^{\varepsilon}\right)}\left[Y_{\tau}^{t, x, i ; u^{\varepsilon}, \beta\left(u^{\varepsilon}\right)}\right]+C \varepsilon \\
& =Y_{t}^{t, x, i ; u^{\varepsilon}, \beta\left(u^{\varepsilon}\right)}+C \varepsilon=J\left(t, x, i ; u^{\varepsilon}, \beta\left(u^{\varepsilon}\right)\right)+C \varepsilon \\
& \leq \operatorname{esssup}_{u \in \mathcal{U}_{t, T}} J(t, x, i ; u, \beta(u))+C \varepsilon, P \text {-a.s. }
\end{aligned}
$$

From the arbitrariness of $\beta \in \mathcal{B}_{t, T}$, we conclude that

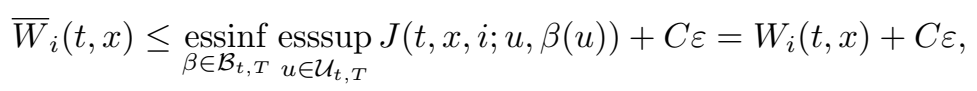

which yields the desired result by letting $\varepsilon \downarrow 0$.

Lemma A.5. For all $i \in \mathbf{K},(t, x) \in[0, T] \times \mathbb{R}$, it holds $\bar{W}_{i}(t, x) \geq W_{i}(t, x)$, P-a.s.

Proof. From the definition of $\bar{W}_{i}(t, x)$ and the standard arguments, we know that for any $\varepsilon>0$, there exists $\beta_{1}^{\varepsilon} \in \mathcal{B}_{t, \tau}$ such that for all $u_{1} \in \mathcal{U}_{t, \tau}$,

$$
\bar{W}_{i}(t, x) \geq G_{t, \tau}^{t, x, i ; u_{1}, \beta_{1}^{\varepsilon}\left(u_{1}\right)}\left[W_{N_{\tau}^{t, i}}\left(\tau, \bar{X}_{\tau}^{t, x, i ; u_{1}, \beta_{1}^{\varepsilon}\left(u_{1}\right)}\right)\right]-\varepsilon, P \text {-a.s. }
$$

We now give the estimate of $W_{N_{\tau}^{t, i}}\left(\tau, \bar{X}_{\tau}^{t, x, i ; u_{1}, \beta_{1}^{\varepsilon}\left(u_{1}\right)}\right)$. It follows from Proposition 3.16 with choosing $\eta=$ $\bar{X}_{\tau}^{t, x, i ; u_{1}, \beta_{1}^{\varepsilon}\left(u_{1}\right)}$ that

$$
W_{N_{\tau}^{t, i}}\left(\tau, \bar{X}_{\tau}^{t, x, i ; u_{1}, \beta_{1}^{\varepsilon}\left(u_{1}\right)}\right)=\operatorname{essinf}_{\beta_{2} \in \mathcal{B}_{\tau, T}} \operatorname{esssup}_{u_{2} \in \mathcal{U}_{\tau, T}} J\left(\tau, \bar{X}_{\tau}^{t, x, i ; u_{1}, \beta_{1}^{\varepsilon}\left(u_{1}\right)}, N_{\tau}^{t, i} ; u_{2}, \beta_{2}\left(u_{2}\right)\right), P \text {-a.s. }
$$

Using a similar method, we deduce that there exists $\beta_{2}^{\varepsilon} \in \mathcal{B}_{\tau, T}$ (depending on $u_{1}$ ) such that for all $u_{2} \in \mathcal{U}_{\tau, T}$,

$$
W_{N_{\tau}^{t, i}}\left(\tau, \bar{X}_{\tau}^{t, x, i ; u_{1}, \beta_{1}^{\varepsilon}\left(u_{1}\right)}\right) \geq J\left(\tau, \bar{X}_{\tau}^{t, x, i ; u_{1}, \beta_{1}^{\varepsilon}\left(u_{1}\right)}, N_{\tau}^{t, i} ; u_{2}, \beta_{2}^{\varepsilon}\left(u_{2}\right)\right)-\varepsilon, P \text {-a.s. }
$$

For $u \in \mathcal{U}_{t, T}$, we define

$$
\beta^{\varepsilon}(u):=\beta_{1}^{\varepsilon}\left(u_{1}\right) \oplus \beta_{2}^{\varepsilon}\left(u_{2}\right)
$$


where $u_{1}=\left.u\right|_{[t, \tau]}, u_{2}=\left.u\right|_{(\tau, T]}$. Then $\beta^{\varepsilon} \in \mathcal{B}_{t, T}$.

Indeed, let $S: \Omega \rightarrow[t, T]$ be an $\mathbb{F}$-stopping time and $u, \tilde{u} \in \mathcal{U}_{t, T}$ be such that $u \equiv \tilde{u}$ on $[[t, S]]$. Decomposing $u, \tilde{u}$ into $u_{1}, \tilde{u}_{1} \in \mathcal{U}_{[t, \tau]}, u_{2}, \tilde{u}_{2} \in \mathcal{U}_{(\tau, T]}$ such that $u=u_{1} \oplus u_{2}, \tilde{u}=\tilde{u}_{1} \oplus \tilde{u}_{2}$. Then we have $u_{1}=\tilde{u}_{1}$ on $[[t, S \wedge \tau]]$ which yields that $\beta_{1}^{\varepsilon}\left(u_{1}\right)=\beta_{1}^{\varepsilon}\left(\tilde{u}_{1}\right)$ on $[[t, S \wedge \tau]]$ since $\beta_{1}^{\varepsilon}$ is nonanticipating. On the other hand, we have $u_{2}=\tilde{u}_{2}$ on ]] $\tau, S \vee \tau]]$ which gives that $\beta_{2}^{\varepsilon}\left(u_{2}\right)=\beta_{2}^{\varepsilon}\left(\tilde{u}_{2}\right)$ on ]] $\left.\left.\tau, S \vee \tau\right]\right]$. Therefore, we get $\beta^{\varepsilon}(u):=\beta_{1}^{\varepsilon}\left(u_{1}\right) \oplus \beta_{2}^{\varepsilon}\left(u_{2}\right)=$ $\beta_{1}^{\varepsilon}\left(\tilde{u}_{1}\right) \oplus \beta_{2}^{\varepsilon}\left(\tilde{u}_{2}\right)=\beta^{\varepsilon}(\tilde{u})$ on $[[t, S]]$, from which it follows that $\beta^{\varepsilon} \in \mathcal{B}_{t, T}$.

Let $u \in \mathcal{U}_{t, T}$ be arbitrarily given and decomposed into $u_{1}=\left.u\right|_{[t, \tau]} \in \mathcal{U}_{t, \tau}$ and $u_{2}=\left.u\right|_{(\tau, T]} \in \mathcal{U}_{\tau, T}$. Then from (A.9), (A.10) and Corollary 3.1 in [6], we get

$$
\begin{aligned}
& \bar{W}_{i}(t, x) \geq G_{t, \tau}^{t, x, i ; u_{1}, \beta_{1}^{\varepsilon}\left(u_{1}\right)}\left[J\left(\tau, \bar{X}_{\tau}^{t, x, i ; u_{1}, \beta_{1}^{\varepsilon}\left(u_{1}\right)}, N_{\tau}^{t, i} ; u_{2}, \beta_{2}^{\varepsilon}\left(u_{2}\right)\right)-\varepsilon\right]-\varepsilon \\
& \geq G_{t, \tau}^{t, x, i ; u_{1}, \beta_{1}^{\varepsilon}\left(u_{1}\right)}\left[J\left(\tau, X_{\tau}^{t, x, i ; u_{1}, \beta_{1}^{\varepsilon}\left(u_{1}\right)}, N_{\tau}^{t, i} ; u_{2}, \beta_{2}^{\varepsilon}\left(u_{2}\right)\right)\right]-C \varepsilon \\
& =G_{t, \tau}^{t, x, i ; u, \beta^{\varepsilon}(u)}\left[Y_{\tau}^{t, x, i ; u, \beta^{\varepsilon}(u)}\right]-C \varepsilon \\
& =Y_{t}^{t, x, i ; u, \beta^{\varepsilon}(u)}-C \varepsilon=J\left(t, x, i ; u, \beta^{\varepsilon}(u)\right)-C \varepsilon, P \text {-a.s. }
\end{aligned}
$$

Consequently, it follows from the arbitrariness of $u \in \mathcal{U}_{t, T}$ that

$$
\bar{W}_{i}(t, x) \geq \underset{u \in \mathcal{U}_{t, T}}{\operatorname{esssup}} J\left(t, x, i ; u, \beta^{\varepsilon}(u)\right)-C \varepsilon \geq \underset{\beta \in \mathcal{B}_{t, T}}{\operatorname{essinf}} \operatorname{esssup}_{u \in \mathcal{U}_{t, T}} J(t, x, i ; u, \beta(u))-C \varepsilon=W_{i}(t, x)-C \varepsilon, \text { P-a.s. }
$$

Finally, letting $\varepsilon \downarrow 0$ we get $\bar{W}_{i}(t, x) \geq W_{i}(t, x)$.

\section{REFERENCES}

[1] G. Barles, R. Buckdahn and E. Pardoux, Backward stochastic differential equations and integral-partial differential equations. Stoch. Stoch. Rep. 60 (1997) 57-83.

[2] R. Buckdahn and Y. Hu, Probabilistic interpretation of a coupled system of Hamilton-Jacobi-Bellman equations. J. Evol. Equ. 10 (2010) 529-549.

[3] R. Buckdahn, Y. Hu and J. Li, Stochastic representation for solutions of Isaacs' type integral-partial differential equations. Stochastic Proc. Appl. 121 (2011) 2715-2750.

[4] R. Buckdahn and J. Li, Stochastic differential games and viscosity solutions of Hamilton-Jacobi-Bellman-Isaacs equations. SIAM J. Control Optim. 47 (2008) 444-475.

[5] J. Li and Q.M. Wei, Optimal control problems of fully coupled FBSDEs and viscosity solutions of Hamilton-Jacobi-Bellman equations. SIAM J. Control Optim. 52 (2014) 1622-1662.

[6] J. Li and Q.M. Wei, $L^{p}$-estimates for fully coupled FBSDEs with jumps. Stoch. Process. Appl. 124 (2014) $1582-1611$.

[7] J. Li and Q.M. Wei, Stochastic differential games for fully coupled FBSDEs with jumps. Appl Math Optim. 71 (2015) 411-448.

[8] E. Pardoux, F. Pradeilles and Z. Rao, Probabilistic interpretation of a system of semi-linear parabolic partial differential equations. Ann. Inst. Henri Poincaré Probab. Statist. 33 (1997) 467-490.

[9] S. Peng, A generalized dynamic programming principle and Hamilton-Jacobi-Bellman equation. Stoch. Stoch. Rep. 38 (1992) 119-134.

[10] S. Peng, BSDE and stochastic optimizations, in Topics in Stochastic Analysis, edited by J. Yan, S. Peng, S. Fand and L. Wu. Science Press, Beijing (1997).

[11] M. Royer, Backward stochastic differential equations with jumps and related non-linear expectations. Stoch. Process. Appl. 116 (2006) 1358-1376. 